\title{
A review of strategies, methods and technologies to reduce non-beneficial consumptive water use on farms considering the FAO56 methods
}

\author{
N. Jovanovic ${ }^{\mathrm{a} *}$, L.S. Pereira ${ }^{\mathrm{b}}$, P. Paredes ${ }^{\mathrm{b}}$, I. Pôças ${ }^{\mathrm{c}}$, V. Cantore $^{\mathrm{d}}$, M. Todorovic ${ }^{\mathrm{e}}$ \\ ${ }^{a}$ CSIR, Water Centre, PO Box 320, Stellenbosch, South Africa \\ ${ }^{\mathrm{b}}$ LEAF - Linking Landscape, Environment, Agriculture and Food, Instituto Superior de Agronomia, Universidade de Lisboa, Portugal \\ ${ }^{\mathrm{c}}$ Geo-Space Sciences Research Centre (CICGE), University of Porto, Portugal \\ d Institute of Sciences of Food Production, National Research Council (CNR-ISPA), Bari, Italy \\ ${ }^{\mathrm{e}}$ International Centre for Advanced Mediterranean Agronomic Studies (CIHEAM), Mediterranean Agronomic Institute of Bari, Italy
}

\section{A R T I C L E I N F O}

\section{Keywords:}

Irrigation management

Irrigation methods

Micro-climate regulation

Mulching

Plant conditioners

Remote sensing

Soil management

\begin{abstract}
A B S T R A C T
In the past few decades, research has developed a multitude of strategies, methods and technologies to reduce consumptive water use on farms for adaptation to the increasing incidence of water scarcity, agricultural droughts and multi-sectoral competition for water. The adoption of these water-saving practices implies accurate quantification of crop water requirements with the FAO56 crop coefficient approach, under diverse water availability and management practices. This paper critically reviews notions and means for maintaining high levels of water consumed through transpiration, land and water productivity, and for minimizing non-beneficial water consumption at farm level. Literature published on sound and quantified experimentation was used to evaluate water-saving practices related to irrigation methods, irrigation management and scheduling, crop management, remote sensing, plant conditioners, mulching, soil management and micro-climate regulation. Summary tables were developed on the benefits of these practices, their effects on non-beneficial water consumption, crop yields and crop water productivity, and the directions for adjustment of FAO56 crop coefficients when they are adopted. The main message is that on-farm application of these practices can result in water savings to a limited extent (usually $<20 \%$ ) compared to sound conventional practices, however this may translate into large volumes of water at catchment scale. The need to streamline data collection internationally was identified due to the insufficient number of sound field experiments and modelling work on the FAO56 crop water requirements that would allow an improved use of crop coefficients for different field conditions and practices. Optimization is required for the application of some practices that involve a large number of possible combinations (e.g. wetted area in micro-irrigation, row spacing and orientation, plant density, different types of mulching, in-field water harvesting) and for strategies such as deficit irrigation that aim at balancing water productivity, the economics of production, infrastructural and irrigation system requirements. Further research is required on promising technologies such as plant and soil conditioners, and remote sensing applications.
\end{abstract}

\section{Introduction and concepts of water use and water consumption}

In the last few decades, population growth and pressures for economic development have put much strain on water resources. This has resulted in competition and over-allocation of water resources, deterioration of water quality, increased vulnerability to extreme weather conditions and water scarcity in many regions of the world. Water scarcity is exacerbated during dry spells and droughts, which frequency and severity is expected to increase in many areas due to the impacts of climate change (IPCC, 2014; Spinoni et al., 2020). Agriculture is a major consumer of water worldwide (FAO, 2016) and it needs to implement measures and practices for restricting the use of water, controlling non-beneficial water consumption and protecting the environment (Pereira et al., 2009a).

The implementation of practices and measures for water conservation and water saving is fundamentally important in the management of water resources. In particular, in the management of agricultural water at field level, it is essential to have an accurate account of the water balance as discussed in detail by Perry (2011), Pereira et al. (2012) and Pereira (2017), mainly in the context of the challenges faced by agriculture to respond to the growing demand for food. Water applied to agricultural fields (water use) is consumed by crops through

\footnotetext{
* Corresponding author.

E-mail address: njovanovic@csir.co.za (N. Jovanovic).
} 


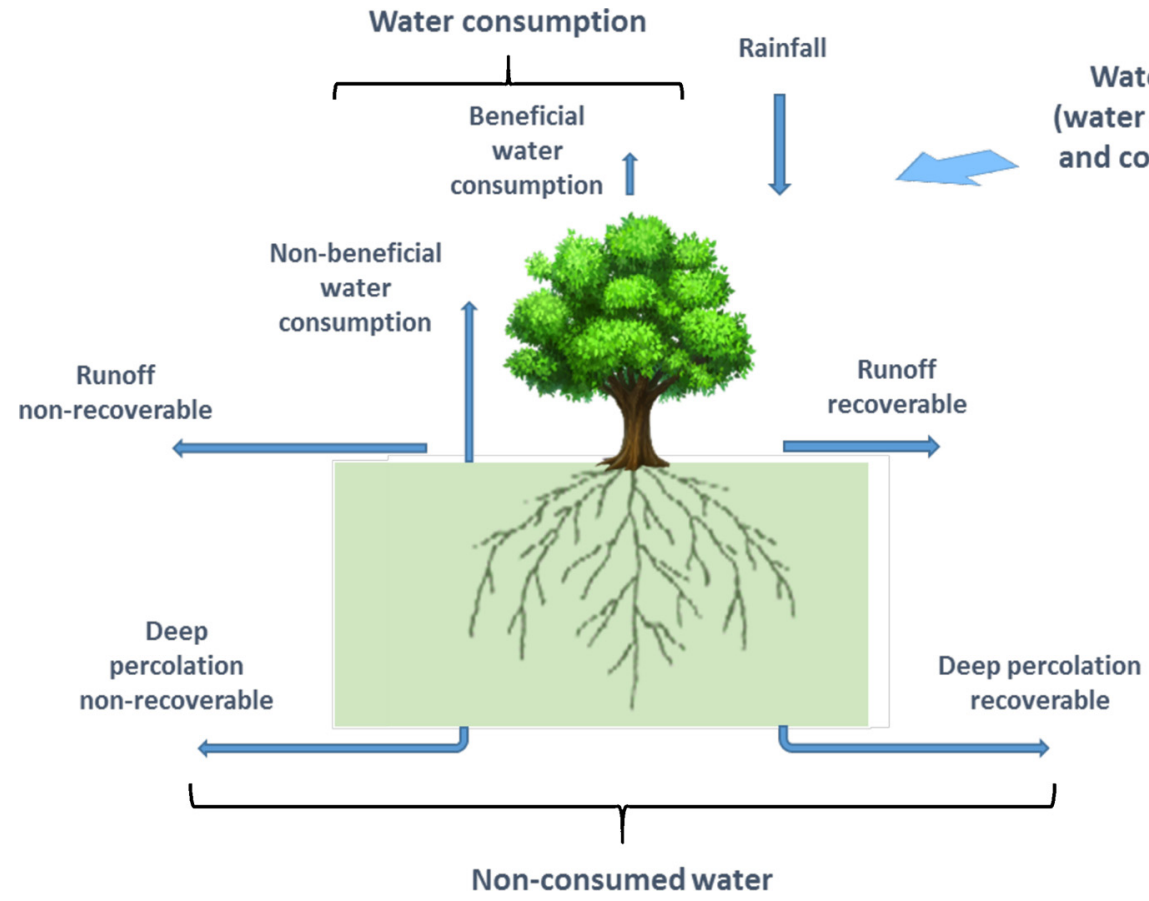

Fig. 1. Partitioning of water use into consumptive use (beneficial and non-beneficial) and non-consumptive use (recoverable and non-recoverable).

evapotranspiration (ET) or it is non-consumed, but may be reusable. Non-consumed water such as runoff (overland flow) and deep percolation (water that flows beyond crops effective root zone) can potentially be recovered by downstream users or contribute to recharge the groundwater, but it can also be non-recoverable. Water consumption can be beneficial when consumed by crops through transpiration, or non-beneficial if consumed by weeds or as soil evaporation (Fig. 1). As reduction in crop transpiration usually implies unwanted reduction in crop yield $\left(\mathrm{Y}_{\mathrm{a}}\right)$, water saving and conservation must logically first target components of the water balance such as the non-beneficial water consumption and non-consumptive water use. Many water-saving and conservation measures and practices (crop, soil and irrigation management) were reviewed and described by Pereira et al. (2009b). In this paper, we focus on strategies, methods and technologies that are available to farmers and practitioners to manipulate and reduce the non-beneficial consumptive water use by application directly at farm level, and in particular how to consider the impacts of related practices when adopting the FAO56 crop water requirements approach.

The FAO56 approach to estimate crop evapotranspiration at the field scale (Allen et al., 1998), which follows up the developments proposed in FAO24 (Doorenbos and Pruitt, 1977), consists of using the $\mathrm{K}_{\mathrm{c}}-\mathrm{ET}_{\mathrm{o}}$ method combining the grass reference evapotranspiration $\left(\mathrm{ET}_{\mathrm{o}}\right.$ ) with a crop coefficient $\left(\mathrm{K}_{\mathrm{c}}\right)$. $\mathrm{ET}_{\mathrm{o}}$ represents the evaporative demand of the atmosphere, and it is thus driven by climate, while $\mathrm{K}_{\mathrm{c}}$ is the ratio $\mathrm{ET}_{\mathrm{c}} / \mathrm{ET}_{\mathrm{o}}$ representing the primary characteristics that distinguish the crop from the grass reference: the crop height, the crop-soil surface resistance and the albedo of the crop-soil surface. In addition to its sound base (Allen et al., 1998; Pereira et al., 1999a), the $\mathrm{K}_{\mathrm{c}}-\mathrm{ET}_{\mathrm{o}}$ method is an internationally tried and tested approach to determine crop water requirements, including the dual $\mathrm{K}_{\mathrm{c}}$ approach (Allen et al., 2005a) as reviewed by Pereira et al. (2015a). The dual $\mathrm{K}_{\mathrm{c}}$ approach, first developed by Wright (1982), provides for the partition of $\mathrm{ET}_{\mathrm{c}}$ into crop transpiration $\left(\mathrm{T}_{\mathrm{c}}\right)$ and soil evaporation $\left(\mathrm{E}_{\mathrm{s}}\right)$, the former estimated through the basal crop coefficient $\left(\mathrm{K}_{\mathrm{cb}}\right)$ and the latter through the soil evaporation coefficient $\left(\mathrm{K}_{\mathrm{e}}\right)$, thus with $\mathrm{K}_{\mathrm{c}}=\mathrm{K}_{\mathrm{cb}}+\mathrm{K}_{\mathrm{e}}$. However, different cultivation strategies, methods and technologies may require adjustments of $K_{c}$ through a stress coefficient $\left(K_{s}\right)$ that adjusts the standard $\mathrm{K}_{\mathrm{c}}$ to the actual $\mathrm{K}_{\mathrm{c}}$, i.e. $\mathrm{K}_{\mathrm{c} \text { act }}=\mathrm{K}_{\mathrm{s}} \mathrm{K}_{\mathrm{c}}$, and $\mathrm{K}_{\mathrm{cb} \text { act }}=\mathrm{K}_{\mathrm{s}} \mathrm{K}_{\mathrm{cb}}$, thus
$\mathrm{K}_{\mathrm{c} \text { act }}=\mathrm{K}_{\mathrm{s}} \mathrm{K}_{\mathrm{cb}}+\mathrm{K}_{\mathrm{e}}$. The stress coefficient may refer to both water and salinity (Allen et al. 1998; Minhas et al., 2020). It becomes evident that reducing non-beneficial water consumption essentially refers to the practices that reduce the $\mathrm{K}_{\mathrm{e}}$ coefficient, rather than $\mathrm{K}_{\mathrm{cb}}$. Typical examples are irrigation methods wetting different portions of the ground (e.g. drip irrigation $v s$. sprinkler), or the manipulation of soil evaporation rates by using different technologies (e.g. organic or plastic mulching).

A basic approach to appropriate use of water is to target irrigations by computing crop water requirements through the product $\mathrm{ET}_{\mathrm{c}}=\mathrm{K}_{\mathrm{c}}$ $\mathrm{ET}_{\mathrm{o}}$ or, anticipating any stress,

$\mathrm{ET}_{\mathrm{c} \text { act }}=\mathrm{K}_{\mathrm{s}} \mathrm{K}_{\mathrm{c}} \mathrm{ET}_{\mathrm{o}}$

or, better, considering the non-consumptive use as soil evaporation

$\mathrm{ET}_{\mathrm{c} \text { act }}=\left(\mathrm{K}_{\mathrm{s}} \mathrm{K}_{\mathrm{cb}}+\mathrm{K}_{\mathrm{e}}\right) \mathrm{ET}_{\mathrm{o}}$

This well-known approach implies appropriate calculation of $\mathrm{ET}_{\mathrm{o}}$ using the Penman-Monteith $\mathrm{ET}_{\mathrm{o}}$ equation (PM-ET $\mathrm{P}_{\mathrm{o}}$, Allen et al., 1998) or an adequate estimation method when full weather data are not available as per the consolidated approaches proposed by Paredes et al. (2020). Alternatively, daily re-analysis weather data may be used to

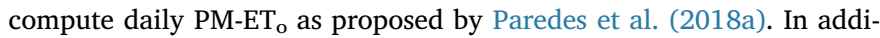
tion, an appropriate selection of the standard crop coefficients and their adjustment to local climate are required. The FAO56 tabulated $\mathrm{K}_{\mathrm{c}}$ and $\mathrm{K}_{\mathrm{cb}}$ were reviewed and updated in the current Special Issue, i.e. Pereira et al. (2020a, b), relative to field and vegetable crops, while the easy estimation of $\mathrm{K}_{\mathrm{cb}}$ and $\mathrm{K}_{\mathrm{c}}$ from the fraction of ground cover and crop height (Allen and Pereira, 2009) is reviewed and $\mathrm{K}_{\mathrm{c}}$ results are tabulated (Pereira et al., 2020c, d). The information provided in Tables in this paper may be used to guide changes of $\mathrm{K}_{\mathrm{c}}$ act or $\mathrm{K}_{\mathrm{cb}}$ act considering the referred tabulated values as upper limits of $\mathrm{K}_{\mathrm{c}}$ and $\mathrm{K}_{\mathrm{cb}}$.

Assuming that farmers already practice good irrigation management (i.e. they don't over-irrigate and they use good irrigation water quality), which novel practices can they adopt to cut further down on water requirements without decreasing crop yield? The first objective of the paper is to answer this question by reviewing practices and measures that minimize non-beneficial water consumption and maintain high levels of water consumed through transpiration, land and water 
Table 1

Improvements in surface irrigation systems that may reduce non-beneficial water consumption and increase consumptive use water productivity (WP $\mathrm{ET}_{\mathrm{ET}}$ ).

\begin{tabular}{|c|c|c|c|}
\hline \multicolumn{2}{|l|}{ Water-saving practices and benefits } & \multicolumn{2}{|l|}{ Potential impacts on actual ET and $\mathrm{WP}_{\mathrm{ET}}$} \\
\hline Water-saving practices & Commonly recognized main benefits & $\begin{array}{l}\text { Potential impacts on non-beneficial water } \\
\text { consumption and } \mathrm{WP}_{\mathrm{ET}}\end{array}$ & $\begin{array}{l}\text { Potential impacts on actual } \mathrm{K}_{\mathrm{c}} \text { and } \\
\text { actual } \mathrm{ET}_{\mathrm{c}}\end{array}$ \\
\hline Precise land levelling ${ }^{1}$ & $\begin{array}{l}\text { Less water to complete advance } \\
\text { phase } \\
\text { Less evaporation from ponded water } \\
\text { Improved distribution uniformity } \\
\text { (DU) }\end{array}$ & $\begin{array}{l}\text { Slightly reduced soil evaporation }\left(\mathrm{E}_{\mathrm{s}}\right) \\
\text { Less total water use } \\
\text { Improved yield due to higher DU } \\
\text { Increased } \mathrm{WP}_{\mathrm{ET}}\end{array}$ & $\begin{array}{l}\text { Slightly smaller } \mathrm{K}_{\mathrm{e}} \\
\mathrm{K}_{\mathrm{cb}} \text { act possibly closer to standard } \\
\mathrm{K}_{\mathrm{cb}} \\
\text { Slightly increased } \mathrm{K}_{\mathrm{c} \text { act }} \text { and } \mathrm{ET}_{\mathrm{c} \text { act }}\end{array}$ \\
\hline Irrigation with anticipated cut-off ${ }^{2}$ & $\begin{array}{l}\text { Reduced water application } \\
\text { Reduced non-beneficial water use }\end{array}$ & $\begin{array}{l}\text { Slightly reduced } \mathrm{E}_{\mathrm{s}} \\
\text { Reduced total water use } \\
\text { Slightly increased } \mathrm{WP}_{\mathrm{ET}}\end{array}$ & $\begin{array}{l}\text { Barely smaller } \mathrm{K}_{\mathrm{e}} \\
\text { Slightly increased } \mathrm{K}_{\mathrm{c} \text { act }} \text { and } \mathrm{ET}_{\mathrm{c} \text { act }}\end{array}$ \\
\hline \multicolumn{4}{|l|}{ Basin and border irrigation $^{3}$} \\
\hline $\begin{array}{l}\text { Higher discharges, reduced widths and/or shorter } \\
\text { lengths }\end{array}$ & $\begin{array}{l}\text { Fast advance phase, reduced } \\
\text { volumes applied } \\
\text { Reduced non-beneficial water use } \\
\text { Higher DU }\end{array}$ & $\begin{array}{l}\text { Barely reduced } \mathrm{E}_{\mathrm{s}} \\
\text { Less total water use } \\
\text { Improved yield due to higher DU } \\
\text { Increased } \mathrm{WP}_{\mathrm{ET}}\end{array}$ & $\begin{array}{l}\text { Slightly smaller } \mathrm{K}_{\mathrm{e}} \\
\mathrm{K}_{\mathrm{cb} \text { act }} \text { slightly closer to } \mathrm{K}_{\mathrm{cb}} \\
\mathrm{K}_{\mathrm{c} \text { act }} \text { approaching standard } \mathrm{K}_{\mathrm{c}} \\
\text { Increased } \mathrm{ET}_{\mathrm{c} \text { act }}\end{array}$ \\
\hline Corrugated basin irrigation for row crops & $\begin{array}{l}\text { Faster advance phase } \\
\text { Reduced non-beneficial water use } \\
\text { Potentially higher DU }\end{array}$ & $\begin{array}{l}\text { Reduced } \mathrm{E}_{\mathrm{s}} \\
\text { Less total water use } \\
\text { Potentially higher } \mathrm{WP}_{\mathrm{ET}}\end{array}$ & $\begin{array}{l}\text { Slightly smaller } \mathrm{K}_{\mathrm{e}} \\
\mathrm{K}_{\mathrm{cb} \text { act }} \text { potentially closer to } \mathrm{K}_{\mathrm{cb}} \\
\text { Slightly increased } \mathrm{K}_{\mathrm{c} \text { act }} \text { and } \mathrm{ET}_{\mathrm{c} \text { act }}\end{array}$ \\
\hline \multicolumn{4}{|l|}{ Rice paddies ${ }^{4}$} \\
\hline Maintaining low water depths in rice basins & $\begin{array}{l}\text { Less total water use } \\
\text { Lower percolation and seepage }\end{array}$ & $\begin{array}{l}\text { Slightly reduced non-beneficial water } \\
\text { consumption and } \mathrm{E}_{\mathrm{S}} \\
\text { Higher total WP } \\
\text { Slightly higher } \mathrm{WP}_{\mathrm{ET}}\end{array}$ & $\begin{array}{l}\text { Barely smaller } \mathrm{K}_{\mathrm{e}} \\
\text { Barely reduced } \mathrm{K}_{\mathrm{c} \text { act }} \\
\text { Slightly smaller } \mathrm{ET}_{\mathrm{c} \text { act }}\end{array}$ \\
\hline $\begin{array}{l}\text { Non-flooded paddies, intermittent irrigation, soil } \\
\text { water near saturation }\end{array}$ & $\begin{array}{l}\text { Less total water use } \\
\text { Lower percolation and seepage } \\
\text { Slightly less evaporation losses }\end{array}$ & $\begin{array}{l}\text { Reduced } \mathrm{E}_{\mathrm{s}} \text { and non-beneficial water } \\
\text { consumption } \\
\text { Higher total WP } \\
\text { Potentially higher } \mathrm{WP}_{\mathrm{ET}}\end{array}$ & 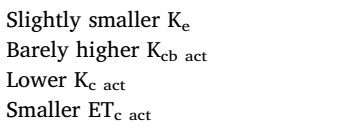 \\
\hline Aerobic rice, surface, sprinkler or drip irrigated ${ }^{3}$ & $\begin{array}{l}\text { Less total water use } \\
\text { Lower percolation and seepage } \\
\text { Less evaporation losses }\end{array}$ & $\begin{array}{l}\text { Reduced } \mathrm{E}_{\mathrm{s}} \text { and non-beneficial water } \\
\text { consumption } \\
\text { Higher total WP due to less water use } \\
\text { (generally less yield) } \\
\text { Potentially higher } \mathrm{WP}_{\mathrm{ET}}\end{array}$ & $\begin{array}{l}\text { Smaller } \mathrm{K}_{\mathrm{e}} \\
\text { Lower } \mathrm{K}_{\mathrm{c} \text { act }} \\
\text { Higher } \mathrm{K}_{\mathrm{cb} \text { act }} / \mathrm{K}_{\mathrm{c} \text { act }} \text { ratio } \\
\text { Smaller } \mathrm{ET}_{\mathrm{c} \text { act }}\end{array}$ \\
\hline \multicolumn{4}{|c|}{ - } \\
\hline Irrigation with alternate furrows ${ }^{4}$ & $\begin{array}{l}\text { Reduced water use } \\
\text { Less evaporation losses due to } \\
\text { smaller wet surface }\end{array}$ & $\begin{array}{l}\text { Reduced } \mathrm{E}_{\mathrm{s}} \\
\text { Higher } \mathrm{WP}_{\mathrm{ET}}\end{array}$ & $\begin{array}{l}\text { Smaller } \mathrm{K}_{\mathrm{e}} \\
\text { Lower } \mathrm{K}_{\mathrm{c} \text { act }} \text { and } \mathrm{ET}_{\mathrm{c} \text { act }}\end{array}$ \\
\hline Surge flow ${ }^{5}$ & $\begin{array}{l}\text { Faster advance phase } \\
\text { Reduced water use } \\
\text { Higher DU }\end{array}$ & $\begin{array}{l}\text { Barely reduced } \mathrm{E}_{\mathrm{s}} \\
\text { Improved yield due to higher DU } \\
\text { Higher } \mathrm{WP}_{\mathrm{ET}}\end{array}$ & $\begin{array}{l}\text { Barely reduced } \mathrm{K}_{\mathrm{e}} \\
\text { Higher } \mathrm{K}_{\mathrm{cb} \text { act }} / \mathrm{K}_{\mathrm{c} \text { act }} \text { ratio } \\
\text { Slightly higher } \mathrm{K}_{\mathrm{c} \text { act }} \text { and } \mathrm{ET}_{\mathrm{c} \text { act }}\end{array}$ \\
\hline Continuously decreased inflow rates (cablegation) ${ }^{6}$ & $\begin{array}{l}\text { Adjusting flow rates to infiltration } \\
\text { Reduced water use } \\
\text { Higher DU }\end{array}$ & 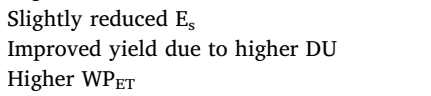 & $\begin{array}{l}\text { Barely reduced } \mathrm{K}_{\mathrm{e}} \\
\text { Higher } \mathrm{K}_{\mathrm{cb} \text { act }} / \mathrm{K}_{\mathrm{c} \text { act }} \text { ratio } \\
\text { Slightly higher } \mathrm{K}_{\mathrm{c} \text { act }} \text { and } \mathrm{ET}_{\mathrm{c} \text { act }}\end{array}$ \\
\hline \multicolumn{4}{|l|}{ Control of water application to the field ${ }^{6}$} \\
\hline $\begin{array}{l}\text { Gated pipes and lay-flat pipes; buried pipes for basins } \\
\text { and borders }\end{array}$ & $\begin{array}{l}\text { Control of inflow rates } \\
\text { Reduced total and non-beneficial } \\
\text { water use }\end{array}$ & $\begin{array}{l}\text { Slightly reduced } \mathrm{E}_{\mathrm{s}} \\
\text { Potentially higher } \mathrm{WP}_{\mathrm{ET}}\end{array}$ & $\begin{array}{l}\text { Barely reduced } \mathrm{K}_{\mathrm{e}} \\
\text { Potentially } \mathrm{K}_{\mathrm{cb} \text { act }} \text { closer to } \mathrm{K}_{\mathrm{cb}} \\
\text { Increased } \mathrm{K}_{\mathrm{c} \text { act }} \text { and } \mathrm{ET}_{\mathrm{c} \text { act }}\end{array}$ \\
\hline Gates in on-farm distribution canals & $\begin{array}{l}\text { Control of inflow rates } \\
\text { Reduced total and non-beneficial } \\
\text { water use }\end{array}$ & $\begin{array}{l}\text { Barely reduced } \mathrm{E}_{\mathrm{s}} \\
\text { Potentially higher } \mathrm{WP}_{\mathrm{ET}}\end{array}$ & $\begin{array}{l}\text { Barely reduced } \mathrm{K}_{\mathrm{e}} \\
\text { Potentially } \mathrm{K}_{\mathrm{cb}} \text { act } \\
\text { Increased } \mathrm{K}_{\mathrm{c} \text { act }} \text { and } \mathrm{ET}_{\mathrm{c} \text { act }}\end{array}$ \\
\hline Automation and control & $\begin{array}{l}\text { Improved operation } \\
\text { Easier irrigation scheduling } \\
\text { Easier adoption of precision } \\
\text { irrigation }\end{array}$ & $\begin{array}{l}\text { Potentially reduced water use } \\
\text { Potentially reduced } \mathrm{E}_{\mathrm{s}} \\
\text { Potentially higher DU and yield } \\
\text { Potentially higher } \mathrm{WP}_{\mathrm{ET}}\end{array}$ & $\begin{array}{l}\text { Slightly smaller } \mathrm{K}_{\mathrm{e}} \\
\text { Favouring } \mathrm{K}_{\mathrm{cb} \text { act }} \text { closer to } \mathrm{K}_{\mathrm{cb}} \\
\text { Favouring higher } \mathrm{K}_{\mathrm{c} \text { act }} \text { and } \mathrm{ET}_{\mathrm{c} \text { act }}\end{array}$ \\
\hline
\end{tabular}

\footnotetext{
1 Fangmeier et al. (1999); Pereira et al. (2003, 2007); Bai et al. (2011, 2017) Walker and Skogerboe (1987); Miao et al. (2015).

2 Pereira et al. (2007); Miao et al. (2015); Morris et al. (2015); Smith and Uddin (2020).

3 Pereira et al. (2003); Morris et al. (2015); Miao et al. (2018); Smith and Uddin (2020).

4 Alberto et al. (2011, 2014) ; Arif et al. (2012); Cesari de Maria et al. (2016); Masseroni, et al. (2017); Sharda et al. (2017).

5 Horst et al. (2007); Gonçalves et al. (2011); Du et al. (2013); Mazarei et al. (2020).

6 Humpherys (1989, 1995); Trout and Kincaid (1989); Niblack and Sanchez (2008); Arnold et al. (2015); Masseroni et al. (2017).
}

productivity, namely the consumptive use water productivity:

$\mathrm{WP}_{\mathrm{ET}}=\mathrm{Y}_{\mathrm{a}} / \mathrm{ET}_{\mathrm{c} \text { act }}$

ratio between actual yield and actual ET. The symbol WP is used when referring to the total water use (TWU), i.e. WP $=\mathrm{Y}_{\mathrm{a}} / \mathrm{TWU}$.

The strategies, methods and technologies reviewed in this paper (hereafter referred jointly as water-saving practices) can aid farmers and practitioners to reduce water use and withdrawal, although sustaining or increasing crop yields, pay less water bills, whilst still maintaining economically sustainable yield. By upscaling to the catchment level, the adoption of these water-saving practices potentially translates into very high volumes of water saved although their impacts may be highly variable (Yan et al., 2015). Although many water-saving practices have been reviewed in the literature (Pereira et al., 2002, 2009a; Bodner et al., 2015; Kang et al., 2017, Perry et al., 2017), quantified data and information based on sound experiments on the practical effects of these practices on the $K_{c}$, mainly on $K_{e}$, are somewhat scarce. Thereafter, the second objective of the paper is to provide qualitative guidelines on the direction for adjustment of FAO56 crop coefficients when these practices are adopted.

The paper is structured in eight sections categorizing practices applicable on farms for reducing non-beneficial water consumption and 
promoting water saving at farm level. Each section of the paper includes: i) a brief description of strategies/methods/technologies, ii) a selection of results from sound literature publications where these strategies/methods/technologies were applied and how they contributed to reducing non-beneficial water consumption and water saving; and iii) summary tables on the benefits and impacts of watersaving practices on $\mathrm{ET}_{\mathrm{c}}, \mathrm{K}_{\mathrm{c}}$ and $\mathrm{WP}_{\mathrm{ET}}$. A wide international literature search was conducted in agricultural water resources and management publications, based on the keywords reflected by the main headings and sub-headings of this paper. In particular, the review publications and papers presenting sound and quantified experimental work that adopt the FAO56 approach were considered. Consolidated summary tables were developed by making use of experimental results published in the literature, theoretical knowledge and experience to indicate the benefits and necessary adjustments of FAO56 crop coefficients under different water-saving practices. The last section synthesizes the outcomes, recommendations and further research required.

\section{Irrigation methods}

Irrigation methods can be broadly classified as surface, sprinkler and micro-irrigation. A consolidated description of irrigation methods and related performance issues was provided by Merriam and Keller (1978), Pereira (1999) and Pereira and Trout (1999a). The basis for field assessment of irrigation performance is provided by Merriam and Keller (1978). Design and management are included in various manuals such as those by Tiercelin and Vidal (2006), Hoffman et al. (2007) and Stetson and Mecham (2011). Contrasting results were found in the literature when water consumption under different irrigation methods were compared, depending on the type of crop, canopy cover, soil properties (e.g. infiltration), land slope, wetted area and irrigation management (Pereira and Trout, 1999b; Thoreson et al., 2013; Perry et al., 2017). This section does not intend to compare different irrigation methods and the implications that occur when more intensive agricultural systems are adopted (Molle and Tanouti, 2017); it rather focuses on water-saving practices that are typical for each irrigation method and applicable on farms. It should be born in mind that costs and benefits play a major role when assessing respective water-saving issues, i.e. economic returns of irrigation tend to decrease when watersaving practices are adopted (Darouich et al. 2014, 2017).

\subsection{Surface irrigation}

Table 1 summarizes practices in surface irrigation systems that may reduce non-beneficial water consumption and increase water productivity. Land levelling for basins and furrows is fundamental for efficient application of surface irrigation methods (Walker and Skogerboe, 1987; Fangmeier et al., 1999; Pereira and Gonçalves, 2018). The science base of surface irrigation practices and models developed in the last 30 years is provided by Walker and Skogerboe (1987). Models (Pereira and Gonçalves, 2018) are not reviewed in the current paper.

As applied water ponds on the land surface before infiltrating into the crop root zone, some evaporation losses are inevitable. Larger irrigation volumes are usually applied at lower frequency compared to other irrigation methods because of operational requirements. This may result in high deep percolation when the soil has high infiltration rates and distribution uniformity is uneven. However, the cumulative soil evaporation may be low as the land surface is wetted less frequently compared to other irrigation methods. These systems can be highly performant when precise laser levelling is adopted, inflow rates are high but non-erosive, and discharge applications are well controlled. These rules apply to either basin or border irrigation (Arnold et al., 2015, Bai et al., 2011, 2017) or to furrow irrigation (Horst et al., 2007). Experiments carried out in Fergana Valley, Uzbekistan, led to reducing water use up to $44 \%$ when surge-flow on alternate furrows was used (Horst et al., 2007). Adopting precision levelling in basin irrigation applied to maize, wheat and sunflower, also adequately controlling inflow rates, allowed achieving a potential water saving of 34 to $39 \%$ (Miao et al., 2015, 2018). However, in both cases, water savings refer mostly to the non-consumed fraction of water use. Adopting alternate furrow irrigation may reduce consumptive water use with little reduction in yield and increased $\mathrm{WP}_{\mathrm{ET}}$ compared to conventional furrow irrigation (Du et al., 2013), including when surge flow is adopted (Horst et al., 2007). However, changing from traditional furrow irrigation to modern water saving practices may be economically non-satisfactory (Darouich et al., 2012).

Advances in the performance of farm surface irrigation were reported on the control of applied water volumes and related discharges, preferably associated with precise land leveling, which allows to close the gap between required and effective application depth (e.g., Horst et al., 2007; Pereira et al., 2007; Morris et al., 2015; Smith and Uddin, 2020). However, achieving appropriate inflow control requires mechanization of water distribution to basins, borders or furrows, preferably automation and remote control (Trout and Kincaid, 1989; Humpherys, 1989, 1995; Pereira et al., 2003; Niblack and Sanchez, 2008; Masseroni et al., 2017). Nevertheless, related research has been declining and mechanized/automated surface irrigation systems are only available in developed countries, mainly in the USA; in other countries, the trend is to progressively replace surface by sprinkler or micro-irrigation supported by strong markets compared to surface irrigation equipment.

Rice is traditionally cultivated in flooded paddy fields where the basins' water depth is around $10 \mathrm{~cm}$. This water plays a double role: providing for crop ET and controlling impacts of temperature on the crop. When land levelling is improved, the flooding depth may be decreased to about $5 \mathrm{~cm}$, which largely decreases deep percolation (DP) (Mao et al., 2004). When climate is favorable, frequent intermittent (nearly daily) flooding may be practiced with the soil water kept near saturation, so further decreasing DP (Arif et al., 2012). When climate is further favorable, infrequent (weekly) flooding may be applied and both DP and $E_{s}$ may decrease. Further control of DP and $E_{s}$ may be obtained with aerobic rice irrigation, including when sprinkler irrigation or drip is adopted (Alberto et al., 2014). However, savings mainly refer to DP, well demonstrated by Cesari de Maria et al. (2016), and very little to the non-beneficial water consumption fraction. Moreover, in irrigated areas where mixed crops are practiced, the resulting decrease in groundwater recharge increases the irrigation demand of other upland crops (Cesari de Maria et al., 2016), which adds to the complexity of water-saving analysis. Nevertheless, the standard $\mathrm{K}_{\mathrm{c}}$ values change with the adopted management of the rice crop as reviewed by Pereira et al. (2020b). As reported, net crop water requirements decrease from conventional flooding to flooding with dry seeding, flooding with anticipated cutoff and, finally, to aerobic rice; however, there is no evidence that $\mathrm{K}_{\mathrm{c}}$ change from permanent flooding to intermittent irrigation since the soil water content is kept near saturation for both management practices.

Less frequent irrigations reduce $\mathrm{E}_{\mathrm{s}}$ compared to more frequent irrigations, but they may increase the risk of crop water stress and $Y_{a}$ reduction when the evaporative demand of the atmosphere $\left(\mathrm{ET}_{\mathrm{o}}\right)$ is very high. The frequency of irrigation wettings depends on various factors, namely the storage capacity of the soil. Soil evaporation is site-specific depending upon climate through $\mathrm{ET}_{\mathrm{o}}$, the frequency of soil wetting, the fraction of ground shadowed by the canopy, which depends upon the crop density and the crop growth stage. It can be quantified through the partition of ET of the considered crop, namely using the dual $\mathrm{K}_{\mathrm{c}}$ approach (Allen et al., 2005a). Examples of application to basin irrigation were provided by Zhao et al. (2013) for wheat and maize, and Wei et al. (2015) for soybean, where the dynamics of $E_{s}$ was simulated and compared with observations. Both examples evidenced the effect of the fraction of soil surface covered by the crop canopy ( $f_{c}$ - canopy cover), with $\mathrm{E}_{\mathrm{s}}$ decreasing until maximum crop growth was attained and increasing afterwards when crop senescence decreased $\mathrm{f}_{\mathrm{c}}$. In other words, 
$E_{s}$ is limited by the shading of the crop canopy, which corresponds to limitations imposed to the available energy for evaporation at the soil surface. This approach is valid for all other irrigation methods that wet the soil surface, in proportion to the wetted fraction as it occurs for furrow irrigation and, particularly, when alternate furrows irrigation is adopted.

\subsection{Sprinkler irrigation}

Sprinkler irrigation systems may be stationary or continuousmoving. The former include portable (non-fixed) set systems, solid set or permanent systems, side roll or wheeled laterals. The latter, automated, consist of center-pivots, linear-move systems, travelling gun sprinklers and side-move systems. These systems are well described and analyzed by Keller and Bliesner (1990) and, in Spanish, by Tarjuelo Martin-Benito (2005), who provided an excellent basis for system design. Nevertheless, the guides and information provided on the internet sites of the manufacturers of sprinklers and equipment are also relevant for design and for efficient use of farm sprinkler systems.

Improved practices that may reduce non-beneficial water consumption and increase $\mathrm{WP}_{\mathrm{ET}}$ in sprinkler irrigation systems are summarized in Table 2 . The water distribution uniformity (DU) throughout the irrigated field is the main objective of system performance (Mantovani et al., 1995). Thus, the considered water-saving practices aim at achieving high DU and controlling wind effects, which also leads to high DU. In addition, practices and design also aim at improving water infiltration and reducing runoff, which can be achieved through appropriate sprinkle selection. Irrigation system design, mainly referring to the selection of sprinklers sizes and their spacing, flow rates, water application depths and diameters of piping, is fundamental to ensure uniform distribution of pressure and water with reduced evaporative losses (Keller and Bliesner, 1990). As water is applied to wet the entire land, non-beneficial consumption of water can be substantial under high wind and evaporative demand of the atmosphere. Wind impacts may be higher when drops are small and when the canopy cover is low and risers are high. Sprinkler systems perform better when the soil infiltration is high and wind is low. Modern sprinkler systems that are designed to frequently apply small irrigation depths, such as center-pivot and linear moving laterals, reduce the risk of crop stress (Rodrigues and Pereira, 2009) at the expense of increased $E_{s}$.

Water losses occur through evaporation from soil and canopy surface. Evaporation losses are difficult to estimate; they mainly depend on wind speed and drift, the evaporative power of the atmosphere estimated by $\mathrm{ET}_{\mathrm{o}}$, canopy cover and the size or the coarseness of irrigation drops. Their control may be achieved by selecting sprinklers and pressure not producing small droplets, avoiding irrigation during the windy and hotter periods of the day (i.e. during the daytime) and reducing the sprinkler height above the canopy. Cavero et al. (2016) found that irrigating by night largely reduces the water losses due to lower wind drift in addition to highly reducing evaporative demand of the atmosphere.

\subsection{Micro-irrigation}

Micro-irrigation systems include high-tech methods with large capital and maintenance investment: surface and subsurface drip irrigation for row crops and orchards, micro-sprinklers and micro-sprayers for horticulture and under-tree irrigation, and bubblers for orchards (Keller and Bliesner, 1990; Lamm et al., 2006; Venot et al., 2019). These low-pressure irrigation systems consist in wetting a limited portion of the ground targeting the crop root zone, which may improve the timeliness of irrigation scheduling and reduce non-beneficial water consumption by reducing $\mathrm{E}_{\mathrm{s}}$. Table 3 summarizes improvements in micro-irrigation systems that may reduce non-beneficial water consumption and increase $\mathrm{WP}_{\mathrm{ET}}$. However, achieving good performances implies adequate design, which complexity may be overcome using decision support models (Pedras and Pereira, 2009). Practices analysed in Table 3 are grouped as aimed at improving water application, the emission uniformity (EU), and the management of irrigation, as well as automation and control. The first two groups essentially refer to design options that may lead to less water use and less costs of investment while leading to higher yields and returns. The last two groups concern the easiness and effectiveness of irrigation management including the avoidance of emitters clogging. References in Table 3 generally refer to more than one aspect since design and management practices are interdependent.

The benefits of drip irrigation compared to the more traditional methods (surface and sprinkler irrigation) include reduced water use, controlled operational water losses, less labour and, comparatively to sprinklers, reduced energy consumption and pumping costs per unit area, as well as improved management of water, fertilization and pesticides for a range of high-profit crops (Perry et al., 2017). Microsprinklers and micro-sprayers have similar benefits, but the wetting pattern is wider and may be affected by wind. However, for under-tree irrigation, wind drift and $\mathrm{E}_{\mathrm{s}}$ are much less than for field crops. Despite advantages, managing drip or micro-spray irrigation systems is very demanding, including when automation is adopted.

This type of high-tech irrigation methods may not be economically feasible under all conditions since, for field crops, economic returns tend to decrease when stricter water-saving techniques are applied (Rodrigues et al., 2013; Darouich et al. 2014; Jacques et al., 2018). Darouich et al. (2014) assessed the use of drip and improved surface irrigation systems for cotton irrigation in Syria and reported that drip irrigation should be selected when water resources are limited, whilst they recommended surface irrigation to secure the highest economic returns. Benefits are generally greater for irrigating tree and vine crops having a better developed root system compared to annual crops, in particular when proper irrigation management is adopted. Water can be easily applied near the root zone of these crops and it makes it easier to apply deficit irrigation, in particular partial root-zone drying (PRD). Soil evaporation is therefore controlled because the soil is only partially wetted near the crops, often under shadow or, when subsurface drip is applied, wetting occurs only by capillarity from the buried emitters. Advantages of drip irrigation for saline environments were recently reviewed by Minhas et al. (2020), such as the reduced accumulation of salts in the root zone and suitability for salt sensitive crops, as well as reduced toxicity and leaf damage due to avoidance of direct contact of water with leaves.

\section{Irrigation management and irrigation scheduling}

The goal of irrigation management and scheduling may differ depending on the purpose. The goal of the farmers is to maximize the economic benefit by increasing yield and/or reducing costs. This can be achieved by maximizing beneficial water consumption and $\mathrm{Y}_{\mathrm{a}}$. As there is a strong relation between $\mathrm{T}_{\mathrm{c}}$ and $\mathrm{CO}_{2}$ assimilation (gas exchange through stomata), higher $\mathrm{Y}_{\mathrm{a}}$ is achieved at the expense of more water transpired. Under conditions of water scarcity, the goal of irrigation management may be to maximize crop $\mathrm{WP}_{\mathrm{ET}}$, i.e. the marketable yield per unit water consumed if such solution is economically feasible. In other words, the economics of production should always be considered by using the economic water productivity ratio (EWPR), which relates the economic benefit to the total cost of water used to achieve a certain crop yield (Pereira et al., 2012). In some instances, the goal of maximizing the economics of production may be achieved by inducing crop water stress to reduce vegetative growth and optimize the quality of marketable yields, especially in some fruit tree crops and vineyards (Fereres and Soriano, 2007).

To save water and maximize $\mathrm{WP}_{\mathrm{ET}}$, an irrigation schedule has to be planned and such plan may or not include deficit irrigation depending upon the crop and water availability. For annual crops, it has to include a selection of the planting date according to the local climate and/or 
Table 2

Improvements in sprinkler irrigation systems that may reduce non-beneficial water consumption and increase consumptive use water productivity (WP $\mathrm{E}_{\mathrm{ET}}$ ).

\begin{tabular}{|c|c|c|c|}
\hline \multicolumn{2}{|l|}{ Water-saving practices and benefits } & \multicolumn{2}{|l|}{ Potential impacts on actual ET and $\mathrm{WP}_{\mathrm{ET}}$} \\
\hline Water-saving practices & Commonly recognized main benefits & $\begin{array}{l}\text { Potential impacts on non-beneficial water } \\
\text { consumption and } \mathrm{WP}_{\mathrm{ET}}\end{array}$ & $\begin{array}{l}\text { Potential impacts on actual } \mathrm{K}_{\mathrm{c}} \\
\text { and actual } \mathrm{ET}_{\mathrm{c}}\end{array}$ \\
\hline \multicolumn{4}{|l|}{ Aimed at improving distribution uniformity $(D U)^{1}$} \\
\hline Optimising overlapping of sprinkler jets & $\begin{array}{l}\text { Less total water use } \\
\text { Improved DU }\end{array}$ & $\begin{array}{l}\text { Reduced } E_{s} \\
\text { Increased yields due to higher DU } \\
\text { Higher } \mathrm{WP}_{\mathrm{ET}}\end{array}$ & $\begin{array}{l}\text { Smaller } \mathrm{K}_{\mathrm{e}} \\
\mathrm{K}_{\mathrm{cb}} \text { act close to standard } \mathrm{K}_{\mathrm{cb}} \\
\text { Higher } \mathrm{K}_{\mathrm{c} \text { act }} \text { and } \mathrm{ET}_{\mathrm{c} \text { act }}\end{array}$ \\
\hline $\begin{array}{l}\text { Minimising discharge variations within the operation } \\
\text { system }\end{array}$ & $\begin{array}{l}\text { Improved DU } \\
\text { Reduced water use }\end{array}$ & $\begin{array}{l}\text { Potentially reduced } \mathrm{E}_{\mathrm{s}} \\
\text { Potentially increased yields } \\
\text { Higher } \mathrm{WP}_{\mathrm{ET}}\end{array}$ & $\begin{array}{l}\text { Slightly reduced } \mathrm{K}_{\mathrm{e}} \\
\mathrm{K}_{\mathrm{cb}} \text { act } \text { closer to standard } \mathrm{K}_{\mathrm{cb}} \\
\text { Potentially higher } \mathrm{K}_{\mathrm{c} \text { act }} \text { and } \\
\mathrm{ET}_{\mathrm{c} \text { act }}\end{array}$ \\
\hline Pressure regulators in sloping and variable slope fields & $\begin{array}{l}\text { Improved DU } \\
\text { Reduced water use }\end{array}$ & $\begin{array}{l}\text { Potentially reduced } E_{s} \\
\text { Potentially increased yields } \\
\text { Slightly higher } \mathrm{WP}_{\mathrm{ET}}\end{array}$ & $\begin{array}{l}\text { Slightly reduced } \mathrm{K}_{\mathrm{e}} \\
\mathrm{K}_{\mathrm{cb}} \text { act } \text { closer to standard } \mathrm{K}_{\mathrm{cb}} \\
\text { Potentially higher } \mathrm{K}_{\mathrm{c} \text { act }} \text { and } \\
\mathrm{ET}_{\mathrm{c} \text { act }}\end{array}$ \\
\hline Pressure control at hydrants & Improved DU & $\begin{array}{l}\text { Potentially increased yields } \\
\text { Slightly higher } \mathrm{WP}_{\mathrm{ET}}\end{array}$ & $\begin{array}{l}\text { Potentially higher } \mathrm{K}_{\mathrm{cb} \text { act }} \\
\text { Potentially higher } \mathrm{K}_{\mathrm{c} \text { act }} \text { and } \\
\mathrm{ET}_{\mathrm{c} \text { act }}\end{array}$ \\
\hline Booster pumps for end gun sprinklers in moving laterals & $\begin{array}{l}\text { Improved DU } \\
\text { Slightly reduced water use }\end{array}$ & $\begin{array}{l}\text { Potentially reduced } \mathrm{E}_{\mathrm{s}} \\
\text { Potentially increased yields } \\
\text { Slightly higher } \mathrm{WP}_{\mathrm{ET}}\end{array}$ & $\begin{array}{l}\text { Barely reduced } \mathrm{K}_{\mathrm{e}} \\
\mathrm{K}_{\mathrm{cb}} \text { act slightly closer to } \mathrm{K}_{\mathrm{cb}} \\
\text { Potentially higher } \mathrm{K}_{\mathrm{c} \text { act }} \text { and } \\
\mathrm{ET}_{\mathrm{c} \text { act }}\end{array}$ \\
\hline \multicolumn{4}{|l|}{ Aimed at controlling wind effects ${ }^{2}$} \\
\hline Irrigation during non-windy periods, at night if possible ${ }^{1}$ & $\begin{array}{l}\text { Minimising wind drift and } \\
\text { evaporation losses } \\
\text { Increased beneficial water use } \\
\text { Improved DU }\end{array}$ & $\begin{array}{l}\text { Reduced } \mathrm{E}_{\mathrm{S}} \\
\text { Higher yields } \\
\text { Higher } \mathrm{WP}_{\mathrm{ET}}\end{array}$ & $\begin{array}{l}\text { Highly reduced } \mathrm{K}_{\mathrm{e}} \\
\mathrm{K}_{\mathrm{cb} \text { act }} \text { closer to standard } \mathrm{K}_{\mathrm{cb}} \\
\text { Higher } \mathrm{K}_{\mathrm{c} \text { act }} \text { and } \mathrm{ET}_{\mathrm{c} \text { act }}\end{array}$ \\
\hline Smaller spacing in windy areas & $\begin{array}{l}\text { Minimising wind drift and } \\
\text { evaporation losses } \\
\text { Increased beneficial water use } \\
\text { Improved DU }\end{array}$ & $\begin{array}{l}\text { Reduced } \mathrm{E}_{\mathrm{s}} \\
\text { Improved yields } \\
\text { Higher } \mathrm{WP}_{\mathrm{ET}}\end{array}$ & $\begin{array}{l}\text { Reduced } \mathrm{K}_{\mathrm{e}} \\
\mathrm{K}_{\mathrm{cb} \text { act }} \text { closer to standard } \mathrm{K}_{\mathrm{cb}} \\
\text { Higher } \mathrm{K}_{\mathrm{c} \text { act }} \text { and } \mathrm{ET}_{\mathrm{c} \text { act }}\end{array}$ \\
\hline Avoid high jet angles in windy areas & $\begin{array}{l}\text { Minimising wind drift } \\
\text { Lower non-beneficial water use } \\
\text { Improved DU }\end{array}$ & $\begin{array}{l}\text { Reduced } \mathrm{E}_{\mathrm{s}} \\
\text { Slightly improved yields } \\
\text { Potentially higher } \mathrm{WP}_{\mathrm{ET}}\end{array}$ & $\begin{array}{l}\text { Reduced } \mathrm{K}_{\mathrm{e}} \\
\mathrm{K}_{\mathrm{cb} \text { act }} \text { closer to standard } \mathrm{K}_{\mathrm{cb}} \\
\text { Slightly higher } \mathrm{K}_{\mathrm{c} \text { act }} \text { and } \mathrm{ET}_{\mathrm{c} \text { act }}\end{array}$ \\
\hline Sprinkler height and sprayers on drop of moving laterals & $\begin{array}{l}\text { Minimising wind drift } \\
\text { Lower non-beneficial water use } \\
\text { Improved DU }\end{array}$ & $\begin{array}{l}\text { Reduced } \mathrm{E}_{\mathrm{s}} \\
\text { Potentially improved yields } \\
\text { Slightly higher } \mathrm{WP}_{\mathrm{ET}}\end{array}$ & $\begin{array}{l}\text { Reduced } \mathrm{K}_{\mathrm{e}} \\
\text { Slightly increased } \mathrm{K}_{\mathrm{cb}} \text { act } \\
\text { Slightly higher } \mathrm{K}_{\mathrm{c} \text { act }} \text { and } \mathrm{ET}_{\mathrm{c} \text { act }}\end{array}$ \\
\hline $\begin{array}{l}\text { Low Energy Precision Application (LEPA) heads in } \\
\text { moving laterals }\end{array}$ & $\begin{array}{l}\text { Minimising wind drift } \\
\text { Lower non-beneficial water use } \\
\text { Improved DU }\end{array}$ & $\begin{array}{l}\text { Reduced } \mathrm{E}_{\mathrm{s}} \\
\text { Potentially improved yields } \\
\text { Slightly higher } \mathrm{WP}_{\mathrm{ET}}\end{array}$ & $\begin{array}{l}\text { Reduced } \mathrm{K}_{\mathrm{e}} \\
\text { Slightly increased } \mathrm{K}_{\mathrm{cb}} \text { act } \\
\text { Slightly higher } \mathrm{K}_{\mathrm{c} \text { act }} \text { and } \mathrm{ET}_{\mathrm{c} \text { act }}\end{array}$ \\
\hline Large sprinkler drops in windy areas & $\begin{array}{l}\text { Minimising wind drift } \\
\text { Lower non-beneficial water use } \\
\text { Improved DU }\end{array}$ & $\begin{array}{l}\text { Reduced } \mathrm{E}_{\mathrm{s}} \\
\text { Slightly improved yields } \\
\text { Slightly higher } \mathrm{WP}_{\mathrm{ET}}\end{array}$ & $\begin{array}{l}\text { Potentially lower } \mathrm{K}_{\mathrm{e}} \\
\text { Barely increased } \mathrm{K}_{\mathrm{cb} \text { act }} \\
\text { Barely increased } \mathrm{K}_{\mathrm{c} \text { act }} \text { and } \mathrm{ET}_{\mathrm{c} \text {-act }}\end{array}$ \\
\hline \multicolumn{4}{|l|}{ Aimed at improving water infiltration and reducing runoff ${ }^{3}$} \\
\hline Sprinkler application rates smaller than infiltration rate & $\begin{array}{l}\text { Maximising water infiltration } \\
\text { Avoiding non-beneficial water uses }\end{array}$ & $\begin{array}{l}\text { Slightly reduced } \mathrm{E}_{\mathrm{s}} \\
\text { Slightly improved yields } \\
\text { Slightly higher } \mathrm{WP}_{\mathrm{ET}}\end{array}$ & $\begin{array}{l}\text { Barely reduced } \mathrm{K}_{\mathrm{e}} \\
\mathrm{K}_{\mathrm{cb} \text { act }} \text { closer to standard } \mathrm{K}_{\mathrm{cb}} \\
\text { Higher } \mathrm{K}_{\mathrm{c} \text { act }} \text { and } \mathrm{ET}_{\mathrm{c} \text { act }}\end{array}$ \\
\hline $\begin{array}{l}\text { Improved spray heads and booms in moving laterals on } \\
\text { heavy soils and sloping land }\end{array}$ & $\begin{array}{l}\text { Maximising water infiltration } \\
\text { Avoiding non-beneficial water uses }\end{array}$ & $\begin{array}{l}\text { Slightly reduced } \mathrm{E}_{\mathrm{s}} \\
\text { Slightly improved yields } \\
\text { Slightly higher } \mathrm{WP}_{\mathrm{ET}}\end{array}$ & $\begin{array}{l}\text { Barely reduced } \mathrm{K}_{\mathrm{e}} \\
\mathrm{K}_{\mathrm{cb} \text { act }} \text { closer to standard } \mathrm{K}_{\mathrm{cb}} \\
\text { Higher } \mathrm{K}_{\mathrm{c} \text { act }} \text { and } \mathrm{ET}_{\mathrm{c} \text { act }}\end{array}$ \\
\hline $\begin{array}{l}\text { Variable rate water application with centre-pivot and } \\
\text { linear systems }\end{array}$ & $\begin{array}{l}\text { Maximising water infiltration } \\
\text { Minimizing spatial variation of } \\
\text { available soil water }\end{array}$ & $\begin{array}{l}\text { Improved yields } \\
\text { Slightly higher } \mathrm{WP}_{\mathrm{ET}}\end{array}$ & $\begin{array}{l}\text { Barely reduced } \mathrm{K}_{\mathrm{e}} \\
\mathrm{K}_{\mathrm{cb} \text { act }} \text { closer to standard } \mathrm{K}_{\mathrm{cb}} \\
\text { Higher } \mathrm{K}_{\mathrm{c} \text { act }} \text { and } \mathrm{ET}_{\mathrm{c} \text { act }}\end{array}$ \\
\hline \multicolumn{4}{|l|}{ Automation and remote control ${ }^{4}$} \\
\hline Automation, remote control & $\begin{array}{l}\text { Easier irrigation scheduling } \\
\text { Easier adoption of water and energy } \\
\text { saving strategies } \\
\text { Easier precise water application }\end{array}$ & $\begin{array}{l}\text { Controlled water use } \\
\text { Potentially reduced } \mathrm{E}_{\mathrm{s}} \\
\text { Potentially improved yields } \\
\text { Potentially higher } \mathrm{WP}_{\mathrm{ET}}\end{array}$ & $\begin{array}{l}\text { Potentially reduced } \mathrm{K}_{\mathrm{e}} \\
\text { Favouring } \mathrm{K}_{\mathrm{cb} \text { act }} \text { closer to } \\
\text { standard } \mathrm{K}_{\mathrm{cb}} \\
\text { Favouring higher } \mathrm{K}_{\mathrm{c} \text { act }} \text { and } \\
\mathrm{ET}_{\mathrm{c} \text { act }}\end{array}$ \\
\hline
\end{tabular}

1 Mantovani et al. (1995); Lamaddalena et al. (2007); Yacoubi et al. (2012).

2 Dechmi et al. (2003); Dukes (2006); Cavero et al. (2016).

3 Valín et al. (2012); Sui and Fisher (2015).

4 Peters and Evett (2008); Zapata et al. (2009); Playán et al. (2014).

the foreseen weather conditions ahead of planting, e.g. studies reported by Paredes et al. (2014) for maize, Paredes et al. (2017) for barley and Paredes et al. (2018b) for potato. That selection requires the knowledge of the relationships between evapotranspiration or transpiration deficit and yield decrease. FAO56 and many authors used the linear relation (Doorenbos and Kassam, 1979):

$\left(1-\mathrm{Y}_{\mathrm{a}} / \mathrm{Y}_{\mathrm{m}}\right)=\mathrm{K}_{\mathrm{y}}\left(1-\mathrm{ET}_{\mathrm{c} \text { act }} / \mathrm{ET}_{\mathrm{c}}\right)$ where $\left(1-\mathrm{Y}_{\mathrm{a}} / \mathrm{Y}_{\mathrm{m}}\right)$ represents the relative yield decrease, (1-ET $\mathrm{T}_{\mathrm{c}}$ act $\mathrm{ET}_{\mathrm{c}}$ ) is the relative crop ET deficit and $\mathrm{K}_{\mathrm{y}}$ is the yield response factor, which is crop specific; $Y_{a}$ and $Y_{m}$ are the crop yields achieved when evapotranspiration is, respectively, $\mathrm{ET}_{\mathrm{c} \text { act }}$ or $\mathrm{ET}_{\mathrm{c}}$.

An update of the $\mathrm{K}_{\mathrm{y}}$ values for a large number of mainly annual crops was tabulated by Minhas et al. (2020). Examples of application of Eq. 3 for searching improved irrigation schedules with support of a simulation model using the FAO56 methodology are those by Pereira 
Table 3

Improvements in micro-irrigation systems that may reduce non-beneficial water consumption and increase consumptive use water productivity (WP $\mathrm{ET}_{\text {). }}$

\begin{tabular}{|c|c|c|c|}
\hline \multicolumn{2}{|l|}{ Water-saving practices and benefits } & \multicolumn{2}{|l|}{ Potential impacts on actual ET and $\mathrm{WP}_{\mathrm{ET}}$} \\
\hline Water-saving practices & Commonly recognized main benefits & $\begin{array}{l}\text { Potential impacts on non-beneficial } \\
\text { water consumption and } \mathrm{WP}_{\mathrm{ET}}\end{array}$ & $\begin{array}{l}\text { Potential impacts on actual } \mathrm{K}_{\mathrm{c}} \\
\text { and actual } \mathrm{ET}_{\mathrm{c}}\end{array}$ \\
\hline \multicolumn{4}{|l|}{ Aimed at improving water application ${ }^{1}$} \\
\hline $\begin{array}{l}\text { Single drip line with short emitters spacing for a double } \\
\text { crop row when row spacing is small }\end{array}$ & $\begin{array}{l}\text { Reduced non-beneficial water use } \\
\text { Favouring the adoption of deficit irrigation } \\
\text { (RDI, Table 4) }\end{array}$ & $\begin{array}{l}\text { Reduced } \mathrm{E}_{\mathrm{s}} \\
\text { Potentially higher } \mathrm{WP}_{\mathrm{ET}}\end{array}$ & $\begin{array}{l}\text { Smaller } \mathrm{K}_{\mathrm{e}} \\
\text { Lower } \mathrm{K}_{\mathrm{cb} \text { act }} \text { and } \mathrm{K}_{\mathrm{c} \text { act }}\end{array}$ \\
\hline Double drip line for an orchard crop row & $\begin{array}{l}\text { Easing water use by the crop } \\
\text { Easing the adoption of deficit irrigation } \\
\text { (PRD, Table 4) }\end{array}$ & $\begin{array}{l}\text { Variable impact on } \mathrm{E}_{\mathrm{s}} \\
\text { Improved water uptake by roots } \\
\text { Potentially higher } \mathrm{WP}_{\mathrm{ET}}\end{array}$ & $\begin{array}{l}\text { Slight variation of } \mathrm{K}_{\mathrm{e}} \\
\mathrm{K}_{\mathrm{cb} \text { act }} \text { closer to standard } \mathrm{K}_{\mathrm{cb}} \\
\text { Higher } \mathrm{K}_{\mathrm{c} \text { act }} \text { and } \mathrm{ET}_{\mathrm{c} \text { act }}\end{array}$ \\
\hline Sprayers in high infiltration soils & Avoiding deep percolation & $\begin{array}{l}\text { Potential increase of } \mathrm{E}_{\mathrm{s}} \\
\text { Improved water uptake by roots } \\
\text { Potentially higher } \mathrm{WP}_{\mathrm{ET}}\end{array}$ & $\begin{array}{l}\text { Potentially increased } \mathrm{K}_{\mathrm{e}} \\
\mathrm{K}_{\mathrm{cb} \text { act }} \text { closer to standard } \mathrm{K}_{\mathrm{cb}} \\
\text { Higher } \mathrm{K}_{\mathrm{c} \text { act }} \text { and } \mathrm{ET}_{\mathrm{c} \text { act }}\end{array}$ \\
\hline Low discharge drippers in low infiltration soils & Avoiding runoff & $\begin{array}{l}\text { Potentially reduced } \mathrm{E}_{\mathrm{s}} \\
\text { Improved uniformity of available soil } \\
\text { water } \\
\text { Potentially higher } \mathrm{WP}_{\mathrm{ET}}\end{array}$ & $\begin{array}{l}\text { Slightly decreased } \mathrm{K}_{\mathrm{e}} \\
\mathrm{K}_{\mathrm{cb} \text { act }} \text { closer to standard } \mathrm{K}_{\mathrm{cb}} \\
\text { Higher } \mathrm{K}_{\mathrm{c} \text { act }} \text { and } \mathrm{ET}_{\mathrm{c} \text { act }}\end{array}$ \\
\hline Subsurface drip irrigation and porous pipes & $\begin{array}{l}\text { Controlled non-beneficial water use } \\
\text { Easing water use by the crop }\end{array}$ & $\begin{array}{l}\text { Reduced } \mathrm{E}_{\mathrm{s}} \\
\text { Improved water uptake by roots with } \\
\text { increased yields } \\
\text { Higher } \mathrm{WP}_{\mathrm{ET}}\end{array}$ & $\begin{array}{l}\text { Reduced } \mathrm{K}_{\mathrm{e}} \\
\mathrm{K}_{\mathrm{cb} \text { act }} \text { closer to standard } \mathrm{K}_{\mathrm{cb}} \\
\text { Higher } \mathrm{K}_{\mathrm{c} \text { act }} \text { and } \mathrm{ET}_{\mathrm{c} \text { act }}\end{array}$ \\
\hline \multicolumn{4}{|l|}{ Aimed at improving emission uniformity $(E U)^{2}$} \\
\hline Short emitter spacing in more permeable soils & Increased EU & $\begin{array}{l}\text { Possibly larger } E_{s} \\
\text { Increased yields due to higher EU } \\
\text { Higher WP } \mathrm{WT}_{\mathrm{ET}}\end{array}$ & $\begin{array}{l}\text { Larger } \mathrm{K}_{\mathrm{e}} \\
\mathrm{K}_{\mathrm{cb} \text { act }} \text { closer to standard } \mathrm{K}_{\mathrm{cb}} \\
\text { Higher } \mathrm{K}_{\mathrm{c} \text { act }} \text { and } \mathrm{ET}_{\mathrm{c} \text { act }}\end{array}$ \\
\hline $\begin{array}{l}\text { Adopt pressure regulators in large sets and in sloping } \\
\text { areas }\end{array}$ & $\begin{array}{l}\text { Increased EU by avoiding pressure } \\
\text { variations in the operating set }\end{array}$ & $\begin{array}{l}\text { Increased yields due to higher EU } \\
\text { Higher } \mathrm{WP}_{\mathrm{ET}}\end{array}$ & $\begin{array}{l}\mathrm{K}_{\mathrm{cb} \text { act }} \text { closer to standard } \mathrm{K}_{\mathrm{cb}} \\
\text { Slightly higher } \mathrm{K}_{\mathrm{c} \text { act }} \text { and } \\
\mathrm{ET}_{\mathrm{c} \text { act }}\end{array}$ \\
\hline $\begin{array}{l}\text { Adopt self-compensating emitters in long and sloping } \\
\text { laterals }\end{array}$ & Increased EU & $\begin{array}{l}\text { Increased yields due to higher EU } \\
\text { Higher } \mathrm{WP}_{\mathrm{ET}}\end{array}$ & $\begin{array}{l}\mathrm{K}_{\mathrm{cb} \text { act }} \text { closer to standard } \mathrm{K}_{\mathrm{cb}} \\
\text { Slightly higher } \mathrm{K}_{\mathrm{c} \text { act }} \text { and } \\
\text { ET }_{\mathrm{c} \text { act }}\end{array}$ \\
\hline Use pressure control at hydrants & $\begin{array}{l}\text { Reduced variability of farm pressure and } \\
\text { discharges in collective irrigation areas }\end{array}$ & $\begin{array}{l}\text { Potentially higher EU at farm level } \\
\text { Potentially higher } \mathrm{WP}_{\mathrm{ET}}\end{array}$ & $\begin{array}{l}\text { Potentially higher } \mathrm{K}_{\mathrm{cb} \text { act }} \\
\text { Potentially slightly higher } \\
\mathrm{K}_{\mathrm{c} \text { act }} \text { and } \mathrm{ET}_{\mathrm{c} \text { act }}\end{array}$ \\
\hline \multicolumn{4}{|l|}{ Aimed at improving irrigation management ${ }^{3}$} \\
\hline Use very frequent irrigation with drippers & $\begin{array}{l}\text { Easier water and fertilizer use by the crop } \\
\text { Avoiding soil cracking } \\
\text { Keeping salts out of the wet bulb }\end{array}$ & $\begin{array}{l}\text { Slightly increased } \mathrm{E}_{\mathrm{s}} \\
\text { Improved water uptake by roots with } \\
\text { increased yields } \\
\text { Higher } \mathrm{WP}_{\mathrm{ET}}\end{array}$ & $\begin{array}{l}\text { Negligible increase of } \mathrm{K}_{\mathrm{e}} \\
\mathrm{K}_{\mathrm{cb} \text { act }} \text { closer to standard } \mathrm{K}_{\mathrm{cb}} \\
\text { Higher } \mathrm{K}_{\mathrm{c} \text { act }} \text { and } \mathrm{ET}_{\mathrm{c} \text { act }}\end{array}$ \\
\hline Use frequent irrigation with sprayers & $\begin{array}{l}\text { Easier water and fertilizer use by the crop } \\
\text { Controlling salts }\end{array}$ & $\begin{array}{l}\text { Slightly increased } \mathrm{E}_{\mathrm{s}} \\
\text { Improved water uptake by roots and } \\
\text { increased yields } \\
\text { Higher } \mathrm{WP}_{\mathrm{ET}}\end{array}$ & $\begin{array}{l}\text { Increase of } \mathrm{K}_{\mathrm{e}} \\
\mathrm{K}_{\mathrm{cb} \text { act }} \text { closer to standard } \mathrm{K}_{\mathrm{cb}} \\
\text { Higher } \mathrm{K}_{\mathrm{c} \text { act }} \text { and } \mathrm{ET}_{\mathrm{c} \text { act }}\end{array}$ \\
\hline Use appropriate filtering and filters locations & $\begin{array}{l}\text { Controlled emitter clogging } \\
\text { Avoiding non-uniformity of emitters' } \\
\text { discharges }\end{array}$ & $\begin{array}{l}\text { Uncertain impacts on } \mathrm{E}_{\mathrm{s}} \\
\text { Higher EU and yields } \\
\text { Potentially higher } \mathrm{WP}_{\mathrm{ET}}\end{array}$ & $\begin{array}{l}\mathrm{K}_{\mathrm{cb} \text { act }} \text { closer to standard } \mathrm{K}_{\mathrm{cb}} \\
\text { Higher } \mathrm{K}_{\mathrm{c} \text { act }} \text { and } \mathrm{ET}_{\mathrm{c} \text { act }}\end{array}$ \\
\hline Use periodical chemical flushing to control drip clogging & $\begin{array}{l}\text { Controlled emitter clogging } \\
\text { Avoiding non-uniformity of emitters' } \\
\text { discharges }\end{array}$ & $\begin{array}{l}\text { Potentially higher EU and yields } \\
\text { Potentially higher } \mathrm{WP}_{\mathrm{ET}}\end{array}$ & $\begin{array}{l}\text { Potentially higher } \mathrm{K}_{\mathrm{cb}} \text { act } \\
\text { Potentially higher } \mathrm{K}_{\mathrm{c} \text { act }} \text { and } \\
\mathrm{ET}_{\mathrm{c} \text { act }}\end{array}$ \\
\hline \multicolumn{4}{|l|}{ Automation and remote control $l^{4}$} \\
\hline Automation and controllers & $\begin{array}{l}\text { Easier irrigation scheduling } \\
\text { Easier adoption of water and energy saving } \\
\text { strategies } \\
\text { Easier precise irrigation }\end{array}$ & $\begin{array}{l}\text { Controlled water use } \\
\text { Negligible impacts on } \mathrm{E}_{\mathrm{s}} \\
\text { Potentially improved yields } \\
\text { Potentially higher } \mathrm{WP}_{\mathrm{ET}}\end{array}$ & $\begin{array}{l}\text { Favouring } \mathrm{K}_{\mathrm{cb}} \text { act } \\
\text { standard } \mathrm{K}_{\mathrm{cb}} \\
\text { Favouring higher } \mathrm{K}_{\mathrm{c} \text { act }} \text { and } \\
\mathrm{ET}_{\mathrm{c} \mathrm{ac}}\end{array}$ \\
\hline
\end{tabular}

\footnotetext{
1 Pedras and Pereira (2009); Thoreson et al. (2013); Tripathi et al. (2014).

2 Colaizzi et al. (2004); Pedras and Pereira (2009); Bush et al. (2016).

3 Enciso-Medina et al. (2011); Chauhdary et al. (2015); Yimam et al. (2020).

4 Li et al. (2018); Panigrahi et al. (2019).
}

et al. (2007, 2009b) considering both salinity and water scarcity, or by Popova and Pereira (2008) when searching crop schedules to cope with drought. Examples of using a dual $\mathrm{K}_{\mathrm{c}}$ water balance model which allowed for the partition of ET into $T_{c}$ and $E_{s}$, thus to use $\left(1-T_{c}\right.$ act $\left./ T_{c}\right)$ in Eq. 3, were applied to define the best planting dates, reduced irrigation and better yields and profits for maize, malt barley and pea for industry (Paredes et al., 2014, 2017; Pereira et al., 2015b). Alternatively, for annual crops, instead of using Eq. 3, a crop growth and yield model may be used, e.g. AquaCrop (Raes et al., 2017), as in the applications reported by Farahani et al. (2009) and Pereira et al. (2015b) respectively for cotton and barley. Using irrigation scheduling modeling, irrigation events may be planned using short and medium range weather forecasts. Examples are the studies by Cai et al. (2009), Lorite et al. (2015) and Paredes et al. (2015) for cereals, and Li et al. (2018) for citrus.

\subsection{Deficit irrigation}

Deficit irrigation was specifically introduced to allow some degree of crop water stress and $\mathrm{Y}_{\mathrm{a}}$ reduction to reduce $\mathrm{E}_{\mathrm{s}}$, water and chemical losses, whilst maintaining economically viable yields (Pereira et al., 2002; Manning et al., 2018). Deficit irrigation can be considered a water-saving strategy where soil water depletion in the root zone goes below the optimum yield threshold and irrigation is applied up to a certain level below field capacity so that occasional rainfall can replenish the soil water storage capacity (Table 4). However, the uneven distribution of rainfall and variable weather conditions pose a risk that 
Table 4

Improvements in irrigation and crop management that may reduce non-beneficial water consumption and increase consumptive use water productivity (WP $\mathrm{E}_{\mathrm{ET}}$.

\begin{tabular}{|c|c|c|c|}
\hline \multicolumn{2}{|l|}{ Water-saving practices and benefits } & \multicolumn{2}{|l|}{ Potential impacts on actual ET and $\mathrm{WP}_{\mathrm{ET}}$} \\
\hline Water-saving practices & Commonly recognized main benefits & $\begin{array}{l}\text { Potential impacts on non-beneficial water } \\
\text { consumption and } \mathrm{WP}_{\mathrm{ET}}\end{array}$ & $\begin{array}{l}\text { Potential impacts on actual } \mathrm{K}_{\mathrm{c}} \text { and } \\
\text { actual } \mathrm{ET}_{\mathrm{c}}\end{array}$ \\
\hline \multicolumn{4}{|l|}{ Irrigation management and scheduling } \\
\hline Deficit irrigation $^{1}$ & $\begin{array}{l}\text { Reduced water use when the available water is } \\
\text { limited }\end{array}$ & $\begin{array}{l}\text { Reduced } \mathrm{E}_{\mathrm{s}} \\
\text { Reduced yields for annual crops } \\
\text { Uncertain changes in yields of tree and vine } \\
\text { crops } \\
\text { Uncertain } \mathrm{WP}_{\mathrm{ET}}\end{array}$ & $\begin{array}{l}\text { Lower } \mathrm{K}_{\mathrm{e}} \\
\text { Lower } \mathrm{K}_{\mathrm{c}} \text { act and } \mathrm{K}_{\mathrm{cb}} \text { act } \\
\text { Lower } \mathrm{ET}_{\mathrm{c} \text { act }}\end{array}$ \\
\hline Regulated Deficit Irrigation (RDI) ${ }^{2}$ & $\begin{array}{l}\text { Reduced water use at selected stages of the } \\
\text { crop cycle }\end{array}$ & $\begin{array}{l}\text { Reduced } \mathrm{E}_{\mathrm{s}} \\
\text { Reduced yields for annual crops } \\
\text { Controlled decrease in yields of tree and vine } \\
\text { crops } \\
\text { Potentially higher } \mathrm{WP}_{\mathrm{ET}}\end{array}$ & $\begin{array}{l}\text { Lower } \mathrm{K}_{\mathrm{e}} \\
\text { Lower } \mathrm{K}_{\mathrm{c} \text { act }} \text { and } \mathrm{K}_{\mathrm{cb} \text { act }} \\
\text { Lower } \mathrm{ET}_{\mathrm{c} \text { act }}\end{array}$ \\
\hline $\begin{array}{l}\text { Partial (alternate) Root-zone Drying } \\
\quad(\text { PRD) }\end{array}$ & Reduced water use for trees and vine crops & $\begin{array}{l}\text { Reduced } \mathrm{E}_{\mathrm{s}} \\
\text { Controlled decrease in yields of tree and vine } \\
\text { crops } \\
\text { Potentially higher } \mathrm{WP}_{\mathrm{ET}}\end{array}$ & $\begin{array}{l}\text { Lower } \mathrm{K}_{\mathrm{e}} \\
\text { Lower } \mathrm{K}_{\mathrm{c} \text { act }} \text { and } \mathrm{K}_{\mathrm{cb} \text { act }} \\
\text { Lower } \mathrm{ET}_{\mathrm{c} \text { act }}\end{array}$ \\
\hline \multicolumn{4}{|l|}{ Crop management ${ }^{4}$} \\
\hline Crop selection & Reduced water use & $\begin{array}{l}\text { Potentially reduced } \mathrm{E}_{\mathrm{S}} \\
\text { Potentially higher } \mathrm{WP}_{\mathrm{ET}}\end{array}$ & $\begin{array}{l}\text { Uncertain } \mathrm{K}_{\mathrm{e}} \\
\text { Lower } \mathrm{K}_{\mathrm{cb} \text { act }} \\
\text { Lower } \mathrm{K}_{\mathrm{c} \text { act }} \text { and } \mathrm{ET}_{\mathrm{c} \text { act }}\end{array}$ \\
\hline Shifting of planting date & $\begin{array}{l}\text { Improved rainfall water use } \\
\text { Decreased irrigation requirements }\end{array}$ & $\begin{array}{l}\text { Uncertain impact on } \mathrm{E}_{\mathrm{s}} \\
\text { Potentially increased yield } \\
\text { Higher } \mathrm{WP}_{\mathrm{ET}}\end{array}$ & $\begin{array}{l}\text { Uncertain } \mathrm{K}_{\mathrm{e}} \\
\text { Uncertain } \mathrm{K}_{\mathrm{cb}} \text { act } \\
\text { Uncertain } \mathrm{K}_{\mathrm{c} \text { act }} \text { and } \mathrm{ET}_{\mathrm{c} \text { act }}\end{array}$ \\
\hline Optimizing nutrients supply & $\begin{array}{l}\text { Improved crop growth and yield } \\
\text { Larger fraction of ground cover }\end{array}$ & $\begin{array}{l}\text { Potentially reduced } \mathrm{E}_{\mathrm{s}} \\
\text { Increased yields } \\
\text { Higher } \mathrm{WP}_{\mathrm{ET}}\end{array}$ & $\begin{array}{l}\text { Potentially reduced } \mathrm{K}_{\mathrm{e}} \\
\mathrm{K}_{\mathrm{cb} \text { act }} \text { closer to standard } \mathrm{K}_{\mathrm{cb}} \\
\text { Higher } \mathrm{K}_{\mathrm{c} \text { act }} \text { and } \mathrm{ET}_{\mathrm{c} \text { act }}\end{array}$ \\
\hline Increasing plant density and canopy size & Increased canopy cover & $\begin{array}{l}\text { Reduced } \mathrm{E}_{\mathrm{s}} \\
\text { Increased yields } \\
\text { Potentially higher } \mathrm{WP}_{\mathrm{ET}}\end{array}$ & $\begin{array}{l}\text { Reduced } \mathrm{K}_{\mathrm{e}} \\
\mathrm{K}_{\mathrm{cb} \text { act }} \text { closer to standard } \mathrm{K}_{\mathrm{cb}} \\
\text { Higher } \mathrm{K}_{\mathrm{c} \text { act }} \text { and } \mathrm{ET}_{\mathrm{c} \text { act }}\end{array}$ \\
\hline Inter-cropping & $\begin{array}{l}\text { Better use of natural resources (light, energy, } \\
\text { water) }\end{array}$ & $\begin{array}{l}\text { Uncertain impacts on } \mathrm{E}_{\mathrm{s}} \\
\text { Potentially higher total yield } \\
\text { Higher combined } \mathrm{WP}_{\mathrm{ET}}\end{array}$ & $\begin{array}{l}\text { Uncertain } \mathrm{K}_{\mathrm{e}} \\
\text { Uncertain } \mathrm{K}_{\mathrm{cb}} \text { act } \\
\text { Uncertain } \mathrm{K}_{\mathrm{c} \text { act }} \text { and } \mathrm{ET}_{\mathrm{c} \text { act }}\end{array}$ \\
\hline \multicolumn{4}{|l|}{ Remote sensing } \\
\hline Remote sensing applications ${ }^{5}$ & $\begin{array}{l}\text { Spatial representation of consumptive water } \\
\text { use and yield } \\
\text { Improved condition for applying precision } \\
\text { agriculture }\end{array}$ & $\begin{array}{l}\text { Potentially controlled } \mathrm{E}_{\mathrm{s}} \\
\text { Potentially higher yields } \\
\text { Potentially higher } \mathrm{WP}_{\mathrm{ET}}\end{array}$ & $\begin{array}{l}\text { Uncertain variation of } \mathrm{K}_{\mathrm{e}} \\
\text { Potentially improved } \mathrm{K}_{\mathrm{cb}} \text { act } \\
\text { Higher } \mathrm{K}_{\mathrm{c} \text { act }} \text { and } \mathrm{ET}_{\mathrm{c} \text { act }}\end{array}$ \\
\hline \multicolumn{4}{|l|}{ Plant conditioners } \\
\hline Anti-transpirants ${ }^{6}$ & $\begin{array}{l}\text { Reduced transpiration } \\
\text { Photosynthesis reduced less than transpiration }\end{array}$ & $\begin{array}{l}\text { Uncertain impact on } \mathrm{E}_{\mathrm{s}} \\
\text { Reduced yields due to less transpiration } \\
\text { Uncertain variation of } \mathrm{WP}_{\mathrm{ET}}\end{array}$ & $\begin{array}{l}\text { Uncertain variation of } \mathrm{K}_{\mathrm{e}} \\
\text { Lower } \mathrm{K}_{\mathrm{cb} \text { act }} \text { and } \mathrm{K}_{\mathrm{c} \text { act }} \\
\text { Lower } \mathrm{ET}_{\mathrm{c} \text { act }}\end{array}$ \\
\hline Bio-stimulants ${ }^{7}$ & $\begin{array}{l}\text { Improved nutrient efficiency } \\
\text { Improved abiotic stress tolerance (and/or crop } \\
\text { quality traits) }\end{array}$ & $\begin{array}{l}\text { Uncertain impact on } \mathrm{E}_{\mathrm{s}} \\
\text { Improved yields } \\
\text { Higher } \mathrm{WP}_{\mathrm{ET}}\end{array}$ & $\begin{array}{l}\text { Uncertain variation of } \mathrm{K}_{\mathrm{e}} \\
\text { Improved } \mathrm{K}_{\mathrm{cb}} \text { act } \\
\text { Higher } \mathrm{K}_{\mathrm{c} \text { act }} \text { and } \mathrm{ET}_{\mathrm{c} \text { act }}\end{array}$ \\
\hline Plant growth regulators ${ }^{8}$ & Improved crop growth & $\begin{array}{l}\text { Uncertain impact on } \mathrm{E}_{\mathrm{s}} \\
\text { Improved yields } \\
\text { Potentially higher } \mathrm{WP}_{\mathrm{ET}}\end{array}$ & $\begin{array}{l}\text { Uncertain variation of } \mathrm{K}_{\mathrm{e}} \\
\text { Improved } \mathrm{K}_{\mathrm{cb}} \text { act } \\
\text { Higher } \mathrm{K}_{\mathrm{c} \text { act }} \text { and } \mathrm{ET}_{\mathrm{c} \text { act }}\end{array}$ \\
\hline
\end{tabular}

1 Rosa et al. (2012b); Giménez et al. (2017); Galindo et al. (2018).

2 Zairi et al. (2003); Rodrigues and Pereira (2009); Rodrigues et al. (2013); Paredes et al. (2014); Pereira et al. (2015b); Phogat et al. (2017).

3 Intrigliolo and Castel (2009); Romero et al. (2013); Kang et al. (2017); Adu et al. (2018).

4 Qiu et al. (2013); Rudnick and Irmak (2014); Miao et al. (2016); Paredes et al. (2017, 2018b).

5 Vuolo et al. (2015b); Campos et al. (2017, 2018); Wang et al. (2020).

6 Boari et al. (2015); Cosic et al. (2015); Faralli et al. (2017); AbdAllah et al. (2018); Mantawy and R.F. (2018).

7 Bittelli et al. (2001); Schiattone (2018).

8 Green et al. (1990); Rizk (2009); Cohen et al. (2019).

may lead to $Y_{a}$ losses (Zairi et al., 2003). To be a sustainable practice, reduction of water consumption in deficit irrigation should be proportionally higher than the reduction in $\mathrm{Y}_{\mathrm{a}}$, so that $\mathrm{WP}_{\mathrm{ET}}\left(\mathrm{kg} \mathrm{m}^{-3}\right)$ is higher and EWPR is positive.

Published research provided inconsistent evidence of performance of deficit irrigation as water-saving strategy. Few studies attempted to estimate directly reduction in soil evaporation $\left(\mathrm{K}_{\mathrm{e}}\right)$ under deficit irrigation, e.g. a modelling study by Phogat et al. (2017) done with the HYDRUS-2D model for a vineyard grown in Australia. Zairi et al. (2003) demonstrated that deficit irrigation may be economically viable under average atmospheric evaporative demand, for cereals when adopting supplemental irrigation, but it is not viable for horticultural crops under severe drought. The study by Paredes et al. (2017) on supplemental irrigated barley showed that under drought conditions a trade-off between economic return, water use, and yield should be used for decision-making. In general, in the years of water shortage, farmers should adopt mild deficit irrigation schedules for less profitable and water stress tolerant crops. To be economically viable, the cost of reduction of water use should be higher than the cost of reduction of market value of $\mathrm{Y}_{\mathrm{a}}$ (Rodrigues and Pereira, 2009; Rodrigues et al., 2013). This is difficult to achieve and deficit irrigation is not always the preferred option by farmers due to the increased risk of both $\mathrm{Y}_{\mathrm{a}}$ and monetary losses. However, exceptions were documented where lower $Y_{a}$ results in better quality fruit and higher profits, e.g. deficit irrigation in grapevine has beneficial effects such as decreased berry size and the farmer gets great benefit from it since it improves wine quality. This was the case 
documented by Romero et al. (2013) with regulated deficit irrigation, and Intrigliolo and Castel (2009) with partial root-zone drying.

\subsection{Regulated deficit irrigation (RDI) and partial root-zone drying (PRD)}

Deficit irrigation can be sustained applying a fixed water reduction strategy throughout the crop season or targeted to some non-critical phenological stages in relation to water stress. Regulated (or controlled, managed) deficit irrigation (RDI) targets certain phenological stages during which plants are less sensitive to water stress (Romero et al., 2013). Partial (or alternate) root-zone drying (PRD) targets the production of the plant hormone abscisic acid (ABA), which reduces leaf expansion and stomatal conductance by stressing one portion of the roots, whilst the other portion of the roots sustains transpiration (Galindo et al., 2018). Published research findings on RDI and PRD are not always consistent (Yan et al., 2015; Kang et al., 2017; Adu et al., 2018) (Table 4). In a comprehensive review by Capra et al. (2008), it was suggested that deficit irrigation and associated practices such as RDI and PRD can be acceptable to reduce capital and operating costs of irrigation and to improve yield quality, only if carried out with precision based on the knowledge of crop responses to water stress. RDI scenarios were designed and observed for maize in Portugal (Paredes et al., 2014). The $\mathrm{E}_{\mathrm{s}} / \mathrm{ET}$ increased in deficit irrigation schedules compared to full irrigation because the water-stressed crop had a lower canopy cover. The high deficit irrigation practiced by the farmer led to the lowest $\mathrm{WP}_{\mathrm{ET}}$ mainly due to the high impacts on maize yields. Milddeficit irrigation scenario led to the highest $\mathrm{WP}_{\mathrm{ET}}$ and economic return (EWPR). Based on the economic analyses, RDI may be justified in water scarce areas to increase profits by increasing the irrigated area.

In general, the applicability of deficit irrigation depends on sitespecific conditions, crops and climatic variables (e.g. rainfall distribution). It is a highly complex practice that involves many variables and uncertain outcomes. Given the large variety of conditions and factors affecting crop water consumption, yields and economic return, optimization modelling and decision-support tools are useful aids to design the most appropriate strategies at specific sites and for specific crops allowing to consider diverse criteria, primarily economic (maximizing profits), but also water-saving (minimizing non-beneficial water consumption) and environmental (minimizing leaching and impacts on the environment). The FAO56 approach can be applied in such modelling exercise in order to determine the effects of deficit irrigation on crop $\mathrm{ET}$, non-beneficial crop water consumption and $\mathrm{WP}_{\mathrm{ET}}$.

\section{Crop management}

Crop management covers a variety of practices, which benefits and effects on non-beneficial crop water consumption, $\mathrm{WP}_{\mathrm{ET}}, \mathrm{K}_{\mathrm{c}}$ act values and $\mathrm{ET}_{\mathrm{c}}$ act are summarized in Table 4. Beneficial and non-beneficial crop water consumption can be manipulated to cope with conditions of water scarcity by choosing crops with short biological cycles that have inherent abilities to develop and reproduce fast (drought escape), crops that have reduced $\mathrm{T}_{\mathrm{c}}$ (small $\mathrm{K}_{\mathrm{cb} \text { act }}$ ), better developed root systems or the ability to adjust water potential (drought avoidance), crops that have the ability to maintain high photosynthetic rates under reduced $\mathrm{T}_{\mathrm{c}}$ or to senesce leaves (drought tolerance) (Pereira et al., 2009a), or woody crops that can take advantage of rainwater availability all year round. In that sense, in areas with limited water availability, crop diversification and use of alternative (emerging, under-utilized) crops and biotechnology may play an important role when economic issues are favorable.

Fallowing, shifting and relocating crops and cropping systems can be a crop management option. In particular, the planting dates may impact on beneficial and non-beneficial crop water consumption in response to climatic factors such as air temperature and rainfall distribution (Paredes et al., 2017). Research has confirmed that the response of crops to water stress depends on critical periods in their phenological stages. Under conditions of water scarcity, irrigation during sensitive phenological stages (e.g. reproductive stage in wheat and maize) is favored ahead of irrigation during less sensitive stages (e.g. vegetative stage) (Doorenbos and Kassam, 1979). Increased $T_{c}$ is not necessarily matched with increased $\mathrm{E}_{\mathrm{s}}$ during sensitive water stress periods when the canopy cover is full (e.g. reproductive stage).

According to Liebig's Law of the minimum, crop growth is constrained by the most limiting factor. Optimal crop growth and $\mathrm{T}_{\mathrm{c}}$ may therefore be reduced under conditions of limited supply of nutrients, fertilization and/or water, or under salinity conditions. This effect can be accounted for in the FAO56 approach either by reducing $\mathrm{K}_{\mathrm{c}}$ and $\mathrm{K}_{\mathrm{cb}}$ coefficients empirically, or by adding a multiplicative stress factor (for salts, nutrients and other stresses) to the water stress factor $\left(\mathrm{K}_{\mathrm{s}}\right)($ Allen et al., 1998; Rudnick and Irmak, 2014; Pereira et al., 2015a). The nutrient or other shortage effects manifest through a reduction of plant growth and canopy cover, and therefore an increase in $\mathrm{K}_{\mathrm{e}}$.

Plant density and canopy size affect the utilization of resources. Reducing stand density makes more resources (e.g. water, nutrients) available to individual plants, in particular under rainfed and extensive agriculture. On the other hand, reduced plant density and canopy size may increase $\mathrm{E}_{\mathrm{s}}$ and, sometimes, surface runoff. In general, drier conditions are more suited to lower planting density. Canopy cover is generally used to split ET into $\mathrm{E}_{\mathrm{s}}$ and $\mathrm{T}_{\mathrm{c}}$, because it approximates the ratio of radiation intercepted by the canopy and incoming solar radiation (Ritchie, 1972). The FAO56 (Allen et al., 1998) provided guidelines for the estimation of $\mathrm{K}_{\mathrm{cb}}$ as a function of the effective fraction of soil surface covered or shaded by vegetation (effective canopy cover $f_{c}$ eff). Moreover, Allen and Pereira (2009) developed an equation to estimate $\mathrm{K}_{\mathrm{cb}}$ and $\mathrm{K}_{\mathrm{c}}$ from the effective fraction of ground cover and crop height, successfully applied to vegetable, field and tree crops. Pereira et al. (2020c, d) reviewed applications of that equation, tested it with field data relative to vegetable, field and tree crops, assessed its use with remote sensing vegetation indices, and finally tabulated $\mathrm{K}_{\mathrm{cb}}$ and $\mathrm{K}_{\mathrm{c}}$ values for a variety of crops and respective characteristics, mainly $f_{c}$ eff and crop heights. It is therefore recommended that site-specific $\mathrm{K}_{\mathrm{cb}}$ be determined with that equation using field observed $\mathrm{f}_{\mathrm{c}}$ and height data, or just estimate $\mathrm{K}_{\mathrm{c}}$ and $\mathrm{K}_{\mathrm{cb}}$ from tabulated values, and/or use models that are able to simulate the physics of radiation interception and partitioning into the energy available for $E_{s}$ and $T_{c}$. This approach is particularly applicable to crops planted in rows such as hedgerow tree orchards, given the large number of combinations of canopy sizes and densities (pruning), plant densities, row distances and orientations, wetted areas of micro-irrigation etc. This type of modelling should be applied at the stage of planning and design of the cropping and irrigation system in order to minimize non-beneficial water consumption. However, it can also be applied in established orchards to assess different combinations of canopy management (pruning) and irrigation systems (e.g. drip vs micro-sprayers), as well as to support planning and management of irrigation scheduling.

Inter-cropping and agro-forestry systems are popular practices to optimize the use of resources (water, nutrients and radiation energy). A higher combined $\mathrm{WP}_{\mathrm{ET}}$ can be achieved in agro-forestry systems compared to mono-cropping because soil water between tree rows is consumed for inter-crop $\mathrm{T}_{\mathrm{c}}$ rather than $\mathrm{E}_{\mathrm{s}}$. These principles were used in the SIMDualKc model (Rosa et al., 2012a, b) to account for inter-cropping (Miao et al., 2016) and for green, active ground cover (Fandiño et al., 2012). The FAO56 publication (Allen et al., 1998) proposed to correct $\mathrm{K}_{\mathrm{c}}$ of individual crops in inter-cropping by weighting for the fraction of the surface covered by each crop. This approach was further developed by Miao et al. (2016) for relay inter-cropping systems of two field crops (wheat-maize and wheat-sunflower) when adopting the dual $\mathrm{K}_{\mathrm{c}}$ approach and using the referred SIMDualKc model. Results showed the advantages of the inter-cropping system relative to mono-cropping. 


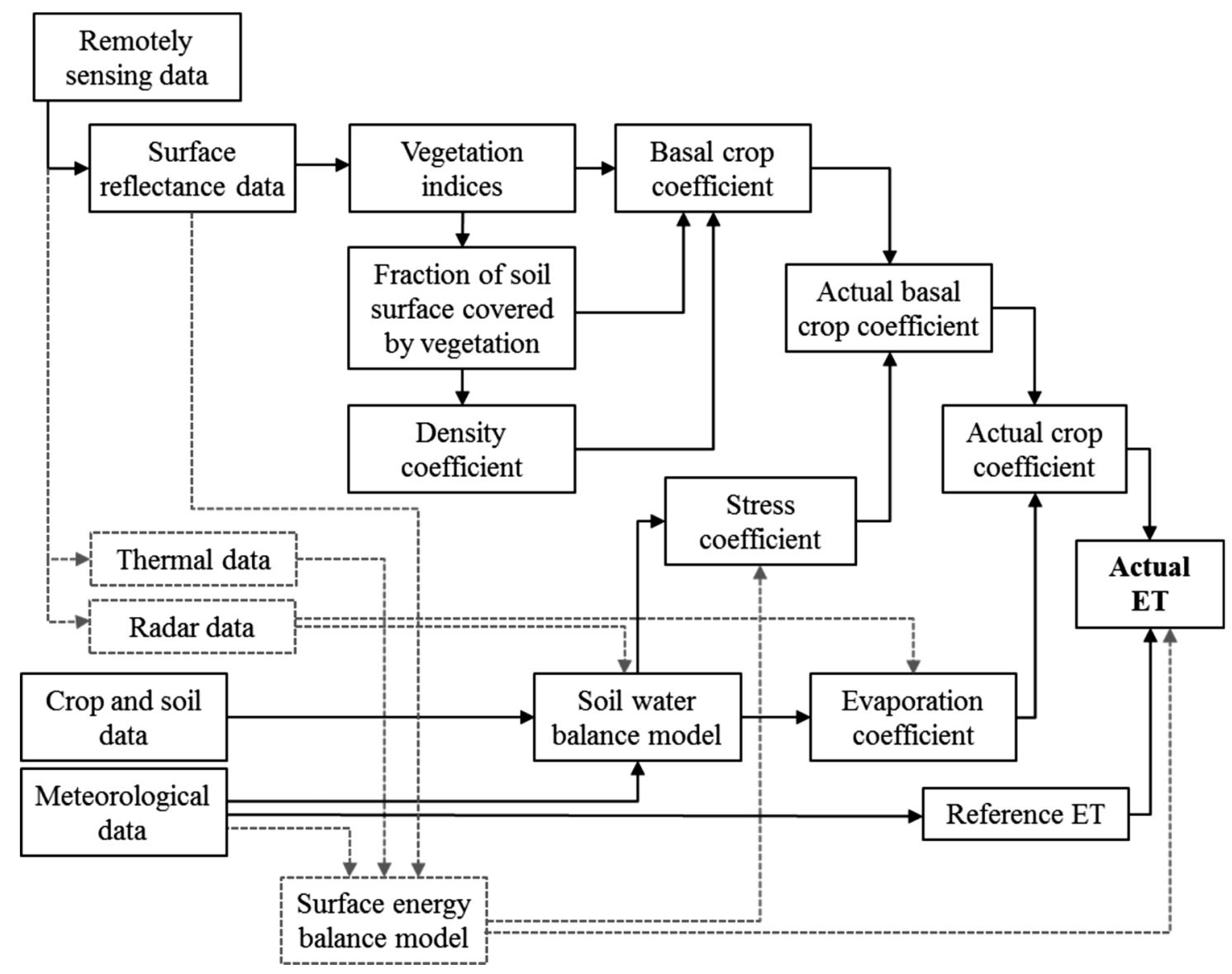

Fig. 2. Flowchart of the remote sensing-based $\mathrm{K}_{\mathrm{c}}-\mathrm{ET}_{\mathrm{o}}$ approach. Dashed lines represent approaches/data that can be combined with $\mathrm{K}_{\mathrm{c}}-\mathrm{ET}_{\mathrm{o}}$ approach.

\section{Remote sensing uses in irrigation management}

Remote sensing (RS) data and methodologies have been increasingly used for estimating parameters related to crop water requirements, e.g. ET and $\mathrm{K}_{\mathrm{cb}}$ (D’Urso et al., 2010; Melton et al., 2012; Vuolo et al., 2015a; Calera et al., 2017). By providing the spatially distributed representation of data, RS allows understanding the within- and among fields variability, which is important to support irrigation management ranging from local farmers up to water-scheme managers (Table 4). The increased number of satellite missions, improved sensors technology, enhanced spatial, temporal and spectral resolutions of data, and easier data access have also encouraged the RS use for irrigation management (Vuolo et al., 2015a; Calera et al., 2017).

In this context, three main RS approaches have been considered for estimating ET: (i) $\mathrm{K}_{\mathrm{c}}-\mathrm{ET}_{\mathrm{o}}$ approach, where reflectance-based actual $\mathrm{K}_{\mathrm{c}}$ or $\mathrm{K}_{\mathrm{cb}}$ coefficients are derived from vegetation indices (VIs) (Pôças et al., 2020); (ii) surface energy balance (SEB) models, combining both spectral and thermal bands data for estimating actual ET as an energy balance residual (e.g., Anderson et al., 2012); and (iii) RS PenmanMonteith techniques, where biophysical parameters (leaf area index LAI, crop height, and surface albedo) are derived from RS data to directly solve the Penman-Monteith equation (e.g. Vuolo et al., 2015a). Due to its simple concept and low input data demand, the RS-based $\mathrm{K}_{\mathrm{c}^{-}}$ $\mathrm{ET}_{\mathrm{o}}$ approach is often preferred for operational use.

Since the 1980's decade, different types of relationships between VIs and $\mathrm{K}_{\mathrm{c} \text { act }}$ or VIs and $\mathrm{K}_{\mathrm{cb}}$ act have been proposed, including linear and non-linear relationships, as well as relationships between $\mathrm{K}_{\mathrm{cb}}$ act and VI integrating canopy cover information derived from RS data, e.g. LAI, $\mathrm{f}_{c}$, and crop density coefficient. The results from three decades of studies have shown a clear relation between VIs and $\mathrm{K}_{\mathrm{cb}}$ act and between VIs and $\mathrm{K}_{\mathrm{c}}$ act for constant rates of soil evaporation. These achievements have paved the way for the use of RS-based $\mathrm{K}_{\mathrm{c}}-\mathrm{ET}_{\mathrm{o}}$ approaches to support irrigation management. A detailed review about reflectancebased $\mathrm{K}_{\mathrm{c} \text { act }}$ or $\mathrm{K}_{\mathrm{cb}}$ act derived from VIs is provided by Pôças et al. (2020).

The normalized difference vegetation index (NDVI) and the soil adjusted vegetation index (SAVI) are the most commonly used VIs for estimating $\mathrm{K}_{\mathrm{cb} \text { act }}$ (or $\mathrm{K}_{\mathrm{c} \text { act }}$ ) due to their relationship with properties of relative abundance of green vegetation. Other VIs can be tested for estimating $\mathrm{K}_{\mathrm{cb}}$ act $\left(\right.$ or $\mathrm{K}_{\mathrm{c} \text { act }}$ ), including the Enhanced Vegetation Index (EVI) and the red edge normalized difference index (red edge NDI), which also present a good relationship with the relative abundance of green vegetation, while having an improved sensitivity at high values of biomass. Nevertheless, for now the use of these two latter VIs is not considered in operational initiatives. The current possibility of combining reflectance data of several satellite missions (e.g. Landsat 8 and Sentinel-2) allows obtaining a dense time series of VIs throughout the growing season, with suitable temporal and spatial resolutions for adequately describing the $\mathrm{K}_{\mathrm{cb}}$ curve. Additionally, the VIs can easily be interpolated for daily values between satellite overpass dates (Mateos et al., 2013). It is important noticing, however, that reductions in $K_{c b}$ resulting from water stress are not immediately represented in $\mathrm{K}_{\mathrm{cb}}$ act derived from VIs, but when an actual biomass reduction or changes in canopy geometry occur (Gonzalez-Dugo et al., 2009; Campos et al., 2017; Pôças et al., 2020). Also, changes in the phenology that are not directly reflected on biomass or vegetation structure are not well represented by these VIs.

The plant water stress can be assessed by computing $\mathrm{K}_{\mathrm{s}}$ through soil water balance (SWB) simulation models. In complement, the actual ET derived from SEB models can be used for assessing ET reduction due to plant water stress (e.g. González-Dugo et al., 2009), though limited to overpass dates of satellites with thermal band (e.g. Landsat missions). 
For now, this latter option is mostly considered in experimental studies instead of operational applications. Additionally, $\mathrm{K}_{\mathrm{e}}$ can be computed through a daily SWB of the soil surface layer, which combined with the $\mathrm{K}_{\mathrm{cb} \text { act }}$ derived from VIs and adjusted for stress with $\mathrm{K}_{\mathrm{s}}$, allows to obtain an actual $K_{c}$. The use of the SWB models requires information about water inputs (precipitation and irrigation) and soil hydraulic properties, combined with $\mathrm{K}_{\mathrm{cb}}$ from VIs, to determine irrigation water requirements (Vuolo et al., 2015a; Calera et al., 2017). Fig. 2 presents a schematic representation of the RS-based $\mathrm{K}_{\mathrm{c}}-\mathrm{ET}_{\mathrm{o}}$ approach.

Overall, the accurate estimation of $\mathrm{K}_{\mathrm{cb} \text { act }}, \mathrm{K}_{\mathrm{c} \text { act }}$ and actual crop ET assisted by RS, in beforehand known crops, improves irrigation management, and contributes to converge actual $\mathrm{K}_{\mathrm{c}}$ and $\mathrm{K}_{\mathrm{cb}}$ values towards the standard ones as well as to achieving larger $\mathrm{WP}_{\mathrm{ET}}$ and water saving, while minimizing non-beneficial water uses associated to over-irrigation (Vuolo et al., 2015a, b; Campos et al., 2017, 2018). Nevertheless, the availability of cloud-free satellite images with an adequate temporal resolution throughout the irrigation season (average frequency of at least 15 days) is needed for a good application of RS-based methodologies for irrigation management (Vuolo et al., 2015b). Several authors observed more accurate estimation of actual ET and better adjustment of actual irrigation amounts to effective irrigation requirements by using actual $\mathrm{K}_{\mathrm{cb}}$ values derived from VIs instead of using tabularized standard $\mathrm{K}_{\mathrm{cb}}$ due to an improved tuning to actual conditions (Campos et al., 2017). As emphasized by Pereira et al. (2015a), FAO56 tabularized values for lengths of crop growth stages and $\mathrm{K}_{\mathrm{c}}$ or $\mathrm{K}_{\mathrm{cb}}$ represent general tendencies while variations due to local crop varieties, new crop hybrids, cultural practices, year-to-year weather effects, pest and diseases effect should be accounted for, as considered through VIs. The use of cumulative growing degree days (GDD) to replace time durations could help overcoming these difficulties (Campos et al., 2017).

The RS-based technologies are currently being used worldwide for operational applications in irrigation management from field scale up to basin and regional scales, through satellite-based irrigation advisory services and WebGIS platforms (Melton et al., 2012; Vuolo et al., 2015a; Calera et al., 2017). Such RS-based applications rely on time series of satellite image data to provide information, in near-real time, about crop biophysical parameters throughout the crop growing seasons. These operational applications are based on the $\mathrm{K}_{\mathrm{c}}-\mathrm{ET}_{\mathrm{o}}$ approach, the RS Penman-Monteith model, or SEB models. For adequately determining the irrigation water requirements and supporting the irrigation scheduling, the operational applications based both on the $\mathrm{K}_{\mathrm{c}}-\mathrm{ET}_{\mathrm{o}}$ approach and the RS Penman-Monteith model are often connected and/ or integrated into a SWB model.

Examples of operational initiatives based on the $\mathrm{K}_{\mathrm{c}}-\mathrm{ET}_{\mathrm{o}}$ approach using $\mathrm{K}_{\mathrm{cb}}$-VI solutions are helpful to increase the beneficial consumptive uses of irrigation water and include:

- IrriSatSMS system (Irrigation Water Management by Satellite and SMS), developed by Commonwealth Scientific and Industrial Research Organisation (CSIRO, Australia) (Hornbuckle et al., 2009; Hornbuckle, 2014): https://irrisat-cloud.appspot.com/;

- SPIDERwebGIS ${ }^{\circledast}$ (System of Participatory Information, Decision support and Expert knowledge for irrigation River basin water management), developed in the context of the European PLEIADES project (D'Urso et al., 2010) and now operated by AgriSat Iberia: http://www.spiderwebgis.org/ and https://www.agrisat.es/en;

- TOPS-SIMS systems, an operational initiative resulting from Satellite Irrigation Management Support (SIMS) project, combined with NASA's Terrestrial Observation and Prediction System (TOPS) (Melton et al., 2012): http://ec2-54-196-147-232.compute-1. amazonaws.com/dgw/sims/.

An emerging functionality in these operational initiatives is the integration of weather forecast products for estimating $\mathrm{ET}_{\mathrm{o}}$, e.g. those of the European Center for Medium Range Weather Forecast, ECMWF, to provide information on crop water requirements ahead of time. This type of functionality should consider reasonable forecast periods (e.g. up to one week ahead) to ensure accurate estimates of crop water requirement ahead of time that might support improved planning of irrigation scheduling (Calera et al., 2017). The architecture of the TOPSIMS system already considers this functionality by integrating data from the NOAA National Weather Service (NWS) Forecasted Reference Evapotranspiration system (FRET) (Melton et al., 2012). Also, the AGRISAT commercial application already considers this functionality for Spain.

As a complement to the operational initiatives based on the RSbased $\mathrm{K}_{\mathrm{c}}-\mathrm{ET}_{\mathrm{o}}$ approach, VIs derived from hyperspectral data (i.e. data collected by RS sensors encompassing a large amount of spectral bands with fine width) can be used for assessing crop water status (e.g., Pôças et al., 2017) and for an adequate management of deficit irrigation. However, despite the good results obtained, the operational use of these hyperspectral-based techniques for irrigation scheduling is still very limited. Nevertheless, the rising availability and technological advances on hyperspectral sensors, associated to the foreseen lower data acquisition costs, creates good perspectives for future operational applications.

By providing spatially distributed information, RS-based initiatives provide useful information for irrigation management and planning considering the within-field variability. Related issues referring to precision agriculture may therefore be supported, thus contributing to approach irrigation application to the required depths resulting in a control of operational water losses and, particularly, a decrease of the non-beneficial consumptive use fraction (Table 4).

\section{Plant conditioners}

Plant conditioners include anti-transpirants, bio-stimulants and plant growth regulators. They are applied to plants to regulate crop growth and yields, and to mitigate the effects of environmental stresses. Although plant conditioners do not directly reduce non-beneficial crop water consumption, they affect $\mathrm{T}_{\mathrm{c}}$ or the $\mathrm{T}_{\mathrm{c}} / \mathrm{ET}$ ratio and therefore they need to be considered when defining irrigation scheduling. The use of plant conditioners do not change the standard $\mathrm{K}_{\mathrm{c}}$ and $\mathrm{K}_{\mathrm{cb}}$ coefficients but may act similarly to the stress coefficients in defining their actual values $\left(\mathrm{K}_{\mathrm{c} \text { act }}\right.$ and $\left.\mathrm{K}_{\mathrm{cb} \text { act }}\right)$. They may affect soil evaporation when plant conditioners decrease (or increase) the crop cover fraction due to their effects on plants (Table 4). Some plant conditioners (e.g. ABA, chitosans, salicylic acid) are reported in more than one of the three categories described below, on the basis of the main effects attributed to them.

\subsection{Anti-transpirants}

Anti-transpirants (AT) are chemicals and compounds that, when sprayed on the leaves, can form a film that physically prevents/reduces the flow of vapor through the stomata or decreases the stomatal opening, thus limiting transpiration. Therefore, $\mathrm{K}_{\mathrm{c} \text { act }}$ and $\mathrm{K}_{\mathrm{cb}}$ act result reduced in relation to the standard $\mathrm{K}_{\mathrm{ct}}$ and $\mathrm{K}_{\mathrm{cb}}$ values and the consumptive use fraction is also reduced. The setback is that $\mathrm{CO}_{2}$ assimilation is concurrently reduced (Kettlewell et al., 2010), since the stomata regulate both water vapor and $\mathrm{CO}_{2}$ fluxes, thus resulting in a decrease of plant cover $f_{c}$, ground shadow and an increase of soil evaporation. The magnitude of this response is species- or cultivar-dependent (Russo and Díaz-Pérez, 2005) and it can vary greatly depending on AT type and mode of application (Hummel, 1990). The success of different ATs lies in the fact that the relative stomata closure reduces transpiration more than photosynthesis. Indeed, the crop growth is also a function of cell expansion, which, in turn, depends on the maintenance of a high plant water potential. Therefore, ATs increase plant water potential and crop growth in spite of reduced photosynthesis (Davenport et al., 1972).

Based on the mode of action, ATs can be classified into three 
categories (AbdAllah et al., 2018, 2019): i) film forming agrochemicals, ii) reflective materials, and iii) metabolic inhibitors. Film forming agrochemicals (emulsions of wax, latex or plastic, polymers), when sprayed on vegetation, dry out creating a transparent film in contact with the various plant organs. The film coats the leaves, blocks the stomata, and, therefore, limits transpiration (Faralli et al., 2017). Its magnitude depends on the uniformity and thickness of the covering film (Palliotti et al., 2013). Reflective materials, such as kaolin or calcium carbonate, are white and their aqueous suspension, when sprayed on plant surfaces, forms a white coating film with high reflectivity after drying. These materials reduce the loss of water by transpiration both directly and indirectly. The coating white film partially occludes the stomata and, by reflecting solar radiation, reduces energy input to the plant thereby reducing leaf temperatures, resulting in $\mathrm{T}_{\mathrm{c}}$ decline (Cantore et al., 2009; Boari et al., 2015; Cosic et al., 2015; Brillante et al., 2016). Metabolic inhibitors of stomata opening are compounds (ABA, acetyl salicilyc acid, fulvic acids, chitosan) that intervene on the biochemical mechanism of stomatal functioning, prevent the complete opening of stomata, thus reducing the loss of water vapour from the leaf (Iriti et al., 2009).

\subsection{Biostimulants}

Plant biostimulants can be defined as 'any substance or microorganism applied to plants with the aim to enhance nutrition efficiency, abiotic stress tolerance and/or crop quality traits, regardless of their nutrients content' (du Jardin, 2015). The major plant biostimulants are: humic and fulvic acids, protein hydrolysates, seaweed extracts, silicon, chitosan, inorganic compounds ( $\mathrm{Al}, \mathrm{Co}, \mathrm{Na}, \mathrm{Se}$ e $\mathrm{Si}$ ), beneficial fungi (i.e., arbuscular mycorrhizal fungi AMF and Trichoderma spp.) and plant growth-promoting bacteria (Canellas et al., 2015; Colla et al., 2015; Rouphael et al., 2015; Ruzzi and Aroca, 2015). The substances may be single compounds (for example glycine betaine) or groups of compounds of natural origin whose composition and bioactive components are not completely characterized (for example seaweed extracts) (du Jardin, 2015). In addition to the above substances, pesticides are available on the market with a fungicidal action based on strobilurins, which has a complementary biostimulant action demonstrated on different physiological processes of plants including the abiotic stress mitigation. In fact, it seems that strobilurins promote the biosynthesis of $\mathrm{ABA}$ and some enzymes involved in oxidative stress (superoxide-dismutase, catalase, peroxidase) (Liang et al., 2018; Schiattone et al., 2018), which may improve gas exchange and $\mathrm{WP}_{\mathrm{ET}}$, especially under water stress conditions (Cantore et al., 2016; Boari et al., 2019).

The mechanisms of action involved in the mitigation of water stress and in the water relationships of plants are not always known and vary depending on the substance or group of substances that characterizes the biostimulant. In the case of humic substances, this action is ascribed to the stimulation of the biosynthesis of phenolic compounds involved in the secondary metabolism and in a wide range of stress responses (du Jardin, 2015). The glycine betaine, proline and other nitrogen compounds seem to intervene mainly through their antioxidant action (Chen and Murata, 2011). Chitosan indirectly affected the control of the stomatal opening through an ABA-dependent mechanism (Iriti et al., 2009). It may be concluded that biostimulants favour plant growth and the crop cover fraction. Therefore, soil evaporation is decreased, and transpiration likely increases. Since $\mathrm{K}_{\mathrm{c}}$ is tied to the energy available for evaporation (Allen et al., 2011), it is not likely that $K_{c}$ act would increase above the standard $\mathrm{K}_{\mathrm{c}}$, but $\mathrm{K}_{\mathrm{cb}}$ act would increase above the standard $\mathrm{K}_{\mathrm{cb}}$ at the expense of $K_{e}$ decrease because of physiological processes associated with transpiration change.

\subsection{Plant growth regulators}

The term "plant growth regulators (PGRs)" includes both plant hormones, also called natural plant growth regulators or phytohormones, and exogenous plant growth regulators. The former are naturally present in plants while the latter are synthesized in the laboratory and given artificially to the plants. The use of these substances by humans is essentially linked to the chemical control of plant development, aimed at improving production. Regardless of their origin, however, these substances have a mediating function in intercellular communication in higher plants, playing an essential role in plant physiology.

PGRs can be divided into two groups: plant growth promoters (auxins, gibberellins and cytokinins) and bioinhibitors (e.g. ABA, methyljasmonate). Ethylene can be grouped either into the promoters or into the plant inhibitors. Other natural inhibitors are groups of substances of different chemical nature, such as phenols, terpenes, lactones and flavonoids. In most cases, they act as antagonists to auxins, gibberellins and cytokinins, counteracting their action or intervening on their metabolism. Bioinhibitors play an important role in plant responses to wounds and biotic and abiotic stress, and are involved in various growth inhibiting activities such as dormancy and abscission. Growth promoters are involved in cell division, cell enlargement, growth, flowering, fruiting and seed formation (Giannakoula et al., 2012). The use of PGRs, such as gibberellins, cytokinins, auxins or their synthetic compounds, is becoming popular to ensure efficient crop production. Several studies pointed out that application of PGRs enhanced plant growth and crop yield (Ud-deen, 2009; Mostafa and Abou Al-Hamd, 2011). At the same time, these compounds can influence in different ways the parameters affecting transpiration and, therefore, water use and $\mathrm{K}_{\mathrm{cb}}$ (Astacio and van Iersel, 2011; Hutsch and Schubert, 2017). Likely, similarly to biostimulants, if plant growth is stimulated, this results in larger transpiration and crop cover, a higher $\mathrm{K}_{\mathrm{cb} \text { act }}$ and a reduced $\mathrm{K}_{\mathrm{e}}$. On the other hand, substances that reduce plant growth and, therefore, LAI, result in lower transpiration and $\mathrm{K}_{\mathrm{cb}}$ act.

The role that the exogenous application of PGRs can have on transpiration and, consequently, on $\mathrm{K}_{\mathrm{cb}}$ can be both direct and indirect in relation to the type of substance used and the processes involved. For example, the exogenous application of ABA or other PGRs that stimulate the biosynthesis by the plants can have a direct effect on transpiration since $\mathrm{ABA}$ is involved in the control of stomatal opening (Rizk, 2009; Astacio and van Iersel, 2011; Zhang et al., 2016). An indirect effect can be found, however, with the application of retarding substances (i.e.: paclobutrazol, uniconazole, chlorocholine chloride) which, acting as antagonists of auxins, gibberellins and cytokinins, modify the architecture of the plant (i.e. height, number and size of the leaves) and, consequently, transpiration rate (Hutsch and Schubert, 2017; Cohen et al., 2019).

In conclusion, it might be emphasized that plant conditioners are effectively suitable to improve the physiological response of plants to environmental stresses, and, thus, increase and stabilize crop production. The plant conditioners affect directly or indirectly $\mathrm{T}_{\mathrm{c}}$ and, consequently, $\mathrm{K}_{\mathrm{cb}}$ and $\mathrm{K}_{\mathrm{c}}$ (Table 4). However, the type and magnitude of crop response varies in relation to numerous variables (e.g. type of plant conditioner, overall pedo-climatic conditions, type and level of abiotic stress, genotype etc.) and it is very difficult to quantify the effects on transpiration and $\mathrm{K}_{\mathrm{c}}$ correction for each crop and each type of plant conditioner. Therefore, it is recommended that new research on the main plant conditioners focuses more on the quantification of parameters related to water consumption of different crops.

\section{Mulching materials and practices}

Mulching is an old practice of covering the soil surface to reduce surface runoff and soil evaporation, improve crop growth, regulate soil temperature, suppress weeds and improve soil health. Different material can be used for mulching, such as bark chips, straw, grass or other plant material, stones or plastic sheets, and humic substances (Rose et al., 2014). A wide variety of environmental and management factors 
Table 5

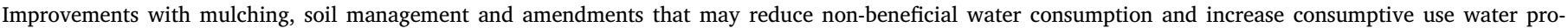
ductivity $\left(\mathrm{WP}_{\mathrm{ET}}\right)$.

\begin{tabular}{|c|c|c|c|}
\hline \multicolumn{2}{|l|}{ Water-saving practices and benefits } & \multicolumn{2}{|l|}{ Potential impacts on actual ET and $\mathrm{WP}_{\mathrm{ET}}$} \\
\hline Water-saving practices & Commonly recognized main benefits & $\begin{array}{l}\text { Potential impacts on non-beneficial water } \\
\text { consumption and } \mathrm{WP}_{\mathrm{ET}}\end{array}$ & $\begin{array}{l}\text { Potential impacts on actual } \mathrm{K}_{\mathrm{c}} \text { and } \\
\text { actual } \mathrm{ET}_{\mathrm{c}}\end{array}$ \\
\hline \multicolumn{4}{|l|}{ Mulching and crop residues ${ }^{1}$} \\
\hline Organic material $^{1}$ & $\begin{array}{l}\text { Improved infiltration } \\
\text { Increased soil water retention }\end{array}$ & $\begin{array}{l}\text { Reduced } E_{s} \\
\text { Higher yields due to increased available soil } \\
\text { water } \\
\text { Higher } \mathrm{WP}_{\mathrm{ET}}\end{array}$ & $\begin{array}{l}\text { Highly reduced } \mathrm{K}_{\mathrm{e}} \\
\text { Potentially higher } \mathrm{K}_{\mathrm{cb} \text { act}} \text {, closer to } \\
\text { standard } \mathrm{K}_{\mathrm{cb}} \\
\text { Lower } \mathrm{K}_{\mathrm{c} \text { act }} \text { and } \mathrm{ET}_{\mathrm{c} \text { act }}\end{array}$ \\
\hline Plastic $^{2}$ & $\begin{array}{l}\text { Controlled soil evaporation } \\
\text { Depending upon soil coverage by plastic sheets, } \\
\text { possibly less water infiltrating the soil } \\
\text { Possible inter-row advective energy transfer }\end{array}$ & $\begin{array}{l}\text { Reduced } \mathrm{E}_{\mathrm{s}} \\
\text { Uncertain impacts on yields } \\
\text { Higher } \mathrm{WP}_{\mathrm{ET}}\end{array}$ & $\begin{array}{l}\text { Highly reduced } \mathrm{K}_{\mathrm{e}} \\
\text { Potentially higher } \mathrm{K}_{\mathrm{cb} \text { act }} \\
\text { Lower } \mathrm{K}_{\mathrm{c} \text { act }} \text { and } \mathrm{ET}_{\mathrm{c} \text { act }}\end{array}$ \\
\hline $\begin{array}{l}\text { Combination of mulching and deficit } \\
\text { irrigation }^{3}\end{array}$ & $\begin{array}{l}\text { Controlled soil evaporation } \\
\text { Uncertain soil water processes }\end{array}$ & $\begin{array}{l}\text { Reduced } E_{s} \\
\text { Reduced yields depending upon the } \\
\text { available soil water } \\
\text { Uncertain variation of } \mathrm{WP}_{\mathrm{ET}}\end{array}$ & $\begin{array}{l}\text { Highly reduced } \mathrm{K}_{\mathrm{e}} \\
\text { Reduced } \mathrm{K}_{\mathrm{cb} \text { act }} \\
\text { Lower } \mathrm{K}_{\mathrm{c} \text { act }} \text { and } \mathrm{ET}_{\mathrm{c} \text { act }}\end{array}$ \\
\hline \multicolumn{4}{|l|}{ Soil management, additives and conditioners } \\
\hline No-tillage and crop residues ${ }^{4}$ & $\begin{array}{l}\text { Improved infiltration } \\
\text { Increased soil water retention }\end{array}$ & $\begin{array}{l}\text { Reduced } E_{s} \\
\text { Uncertain impacts on yields } \\
\text { Higher } \mathrm{WP}_{\mathrm{ET}}\end{array}$ & $\begin{array}{l}\text { Highly reduced } \mathrm{K}_{\mathrm{e}} \\
\text { Uncertain effects on } \mathrm{K}_{\mathrm{cb}} \text { act } \\
\text { Slightly decreased } \mathrm{K}_{\mathrm{c} \text { act }} \text { and } \\
\text { ET }_{\mathrm{c} \text { act }}\end{array}$ \\
\hline In-field water harvesting ${ }^{5}$ & $\begin{array}{l}\text { Improved rainfall water use } \\
\text { Improved infiltration } \\
\text { Increased soil water retention }\end{array}$ & $\begin{array}{l}\text { Uncertain impacts on } \mathrm{E}_{\mathrm{S}} \text { depending upon } \\
\text { crop practices } \\
\text { Increased yields } \\
\text { Higher } \mathrm{WP}_{\mathrm{ET}}\end{array}$ & $\begin{array}{l}\text { Uncertain impacts on } \mathrm{K}_{\mathrm{e}} \\
\text { Higher } \mathrm{K}_{\mathrm{cb}} \text { act } \\
\text { Higher } \mathrm{K}_{\mathrm{c} \text { act }} \text { and } \mathrm{ET}_{\mathrm{c} \text { act }}\end{array}$ \\
\hline Soil additives and conditioners, biochar ${ }^{6}$ & $\begin{array}{l}\text { Improved infiltration } \\
\text { Increased soil water retention }\end{array}$ & $\begin{array}{l}\text { Uncertain impacts on } E_{s} \text { depending upon the } \\
\text { crop and ground cover } \\
\text { Increased yields } \\
\text { Higher } \mathrm{WP}_{\mathrm{ET}}\end{array}$ & $\begin{array}{l}\text { Uncertain impacts on } \mathrm{K}_{\mathrm{e}} \\
\text { Higher } \mathrm{K}_{\mathrm{cb}} \text { act } \\
\text { Higher } \mathrm{K}_{\mathrm{c} \text { act }} \text { and } \mathrm{ET}_{\mathrm{c} \text { act }}\end{array}$ \\
\hline
\end{tabular}

\footnotetext{
1 Unger and Parker (1976); Todd et al. (1991); Klocke et al. (2009); Balwinder-Singh et al (2011); Zribi et al. (2015).

2 Zribi et al. (2015); Fan et al. (2017); Feng et al. (2017); Gong et al. (2017a); He et al. (2019).

3 Yan et al. (2015); Wen et al. (2017).

4 Lampurlanés et al. (2002); Pereira et al. (2003); Schwartz et al. (2010); Morell et al. (2011); DeLaune et al. (2020).

5 Mavimbela and Van Rensburg (2012); Oweis et al. (2012); Daryanto et al. (2017); Grum et al. (2017).

6 Sohi et al. (2010); Andrenelli et al. (2016); Ramos (2017).
}

were reported to be involved in the complex crop growth response to humic substances: application rate, source, crop type, application site, stress and growth media (Rose et al., 2014). Although there is a wealth of information in the literature on mulching (e.g. a Special Issue edited by He et al., 2019), less information is available on how to adjust the actual $\mathrm{K}_{\mathrm{c}}$ and $\mathrm{K}_{\mathrm{cb}}$ to consider mulching effects. The updated $\mathrm{K}_{\mathrm{c}}$ and $\mathrm{K}_{\mathrm{cb}}$ tabulated for vegetable and field crops (Pereira et al. 2020a, b) propose that users consider the effects of mulch in decreasing the actual values of $\mathrm{K}_{\mathrm{c}}$, but not $\mathrm{K}_{\mathrm{cb}}$, taking into account the crop cover fraction. Effects of mulches are greater at earlier crop stages when the soil is uncovered or less covered by the vegetation, thus when crop coefficients also vary greatly with the frequency of wetting and soil wetness; thus, it is proposed that $\mathrm{K}_{\mathrm{c}}$ ini is then estimated according to FAO56 (Allen et al., 1998, 2005b). A consolidation of findings from review papers and studies on various crops is reported in Table 5.

\subsection{Organic mulching}

Impacts of organic mulches on $\mathrm{E}_{\mathrm{s}}$ vary with soil characteristics, climate, mulch quantity and mulch cover fraction, crop cover and crop growth stage among others. Effects of mulches are well described since a few decades (Unger and Parker, 1976; Todd et al., 1991). Yan et al. (2015) reviewed a large number of organic mulching field experiments. They provided average figures of decrease in ET by $3 \%$ for wheat and $4 \%$ for maize with straw mulch and corresponding average increases in $\mathrm{Y}_{\mathrm{a}}$ by $18 \%$ and $5 \%$, respectively. Balwinder-Singh et al. (2011) reported reduced $\mathrm{K}_{\mathrm{c}}$ act from sowing to about 10 days before anthesis, and increased $K_{c}$ act by 0.1-0.2 during the period of maximum $f_{c}$ in rice straw mulch field experiments on wheat. The increased $\mathrm{K}_{\mathrm{c} \text { act }}$ could have been due to less $E_{s}$ and more soil water available to $T_{c}$ rather than an actual change in plant characteristics and $\mathrm{K}_{\mathrm{cb}}$ coefficient. Martins et al. (2013) estimated $E_{s}$ to be less than $9 \%$ of ET in sprinkler and drip irrigated maize with crop residues mulch. Organic mulches and crop residues impact not only on soil evaporation but also on infiltration and soil water storage, in addition to incorporating organic matter in the soil, so having positive effects on carbon sequestration.

\subsection{Plastic mulching}

Yan et al. (2015) indicated, in their review, that plastic mulching reduced ET on average by $11 \%$. In another review study, Ma et al. (2018) generally found an increase of $Y_{a}, W P$ and economic benefit with plastic mulching compared to conventional farming for many crops, and especially for spring maize. Ding et al. (2013) successfully applied the dual crop coefficient approach for assessing the $E_{\mathrm{s}}$ in maize under plastic mulch against micro-lysimeter measurements. A recent study by Feng et al. (2019) reported on the low impacts of plastic film mulch on croplands $K_{c}$ act justifying that the mulch decreases soil evaporation but increases crop transpiration, thus favoring above ground biomass and yield, and leading to a higher $\mathrm{WP}_{\mathrm{ET}}$.

Comparisons between different mulching materials were found in the literature. Zhao et al. (2018) estimated that, based on field experiments and using the HYDRUS-2D model, $\mathrm{E}_{\mathrm{s}}$ can be cut down by 46$52 \%$ with straw mulching in furrows and plastic film in ridges compared to conventional no mulching of wheat; however, such high values are larger than those estimated in other studies, namely reported by $\mathrm{He}$ et al. (2019). Saglam et al. (2017) tested biodegradable paper and plastic mulches on drip-irrigated pumpkin in Washington (USA) using field experiments and modelling. The effects of the biodegradable mulch become less prominent as the material starts degrading 
depending on its properties and weather conditions. The resulting effect is somewhere between no-mulch and non-biodegradable mulch. The advantage is that biodegradable mulch does not need to be removed and disposed after harvest.

Sprayable bio-degradable polymer coatings may represent a more environmentally-friendly alternative to traditional non-degradable polyethylene. A large number of formulations of sprayable bio-degradable materials are available for applications in agriculture (Adhikari et al., 2016). However, more research is required on the polymer properties and mechanisms of degradation, as well as on the interactions between these materials and soil properties.

\subsection{Combination of mulching, deficit irrigation and other practices}

Mulching is often used in combination with other practices, most notably deficit irrigation. For such combination of practices, reduction in ET was documented to be $9 \%$ on average with an increase of wheat $\mathrm{Y}_{\mathrm{a}}$ by $19 \%$, and $6 \%$ with a $\mathrm{Y}_{\mathrm{a}}$ increase of maize by $13 \%$ (Yan et al. 2015), mainly attributable to less $E_{s}$ and more soil water available to $T_{c}$. Combinations of mulching with tillage practices and organic manure were also reported (Wang et al., 2017; Yang et al., 2018a, b).

The original FAO56 (Allen et al., 1998) suggested a reduction in $E_{s}$ of about $5 \%$ for each $10 \%$ of soil surface that is covered by the organic mulch. This approach was successfully applied in SIMDualKc applications (Martins et al., 2013). Allen et al. (1998) also indicated that $\mathrm{E}_{\mathrm{s}}$ may decrease $50-80 \%$ with a resulting increase of $10-30 \%$ in $\mathrm{T}_{\mathrm{c}}$ (more water available to the plant) under mulching compared to no-mulch. However, Allen et al. (1998) also recommended local calibration given the large number of possible combinations of width of the mulching strip, canopy cover, mulching materials etc. Wen et al. (2017) developed an optimization model for deficit irrigation scheduling of spring wheat with mulching. Fan et al. (2017) calculated empirical quadratic equations to reduce ET (and crop coefficients) from plastic mulching of maize as a function of a non-mulching treatment. An alternative to empirical equations that are site-specific and require time-consuming experimental work is to use more mechanistic models such as SIMDualKc (Rosa et al., 2012a, b). The principles adopted in the SIMDualKc model account for a number of input parameters: i) spacing between crop rows, spacing between holes along a row, hole diameter, fraction of soil covered by the plastic film, number of crop rows per plastic sheet, for plastic mulching; and ii) mulch density, fraction of soil covered by the mulch and percentage of reduction in $E_{s}$ for organic mulching. Because of the wide variety of results obtained depending on specific conditions, modelling with calibrated models is recommended to facilitate planning and design of mulching practices (material, size, full/partial mulching and combinations with other practices) in order to minimize non-beneficial water consumption.

\section{Soil management, soil additives and conditioners}

Soil management practices that may modify the volume of water infiltrated and stored in the soil, thereby affecting the ratio of $\mathrm{E}_{\mathrm{s}}$ and $\mathrm{T}_{\mathrm{c}}$, are usually related to tillage (deep, conservation or no-tillage), land preparation (levelling or ridge and furrows) or in-field rainwater harvesting. Soil additives and conditioners are materials used to increase the soil water and nutrients retention capacity or reduce $E_{s}$. Many of these practices affect particularly the top soil, which is the most dynamic soil layer in terms of mass and energy exchanges. Consolidated findings related to these practices are reported in Table 5.

\subsection{Soil tillage}

Conservation tillage or no-tillage is practiced to improve soil water retention capacity and reduce $E_{s}$ through no- or minimum tillage (Kassam et al., 2014). The benefits of conservation agriculture materialize mainly in soils with high water holding capacity, intensive cropping with increased nutrient availability and long cropping cycles. The constraints are mainly the increased risk of deep percolation, especially on soils with low water holding capacity and under relatively low nutrient availability.

However, very little quantifiable published data can be found on the actual water savings that can be achieved with no-tillage, in particular through enhanced water infiltration and by limiting $E_{s}$ with a mulch of crop residues on the soil surface. Lampurlanés et al. (2002) demonstrated that no-tillage with residue from the previous crop can significantly reduce $E_{s}$ in a barley-fallow system compared to conventional tillage, depending on the season. Morell et al. (2011) confirmed that notillage with corrected fertilization rates can be a long-term sustainable practice in dryland agroecosystems, leading to improved crop yields without increase in ET, which is evidence of a more favorable partitioning into $E_{s}$ and $T_{c}$, thus with increased $K_{c b}$ act at the expense of $K_{e}$ and stable $\mathrm{K}_{\mathrm{c}}$ (Table 5 ).

\subsection{Land preparation and in-field water harvesting}

Water harvesting techniques for agriculture (Oweis et al., 2012) include both capturing and storing water in dams or reservoirs, as well as capturing and augmenting water in the soil. The latter refers to infield harvesting that is used to channel even small rainfall of low intensity into planting pits, contour trenches, furrows and terraces. Bouma et al. (2016) reviewed rainwater harvesting in semi-arid Africa and Asia, concluding that this practice can improve crop yields, in particular on rainfed small-holder farms. Advantages of rainwater harvesting were reviewed by Pereira et al. (2009a) and Oweis et al. (2012), among others.

Land preparation for in-field water harvesting is often used in combination with other practices. Grum et al. (2017) demonstrated how in-field rainwater harvesting in combination with straw mulch augments soil water content by reducing water losses via runoff and $E_{s}$ to enable a more resilient environment to crops. Daryanto et al. (2017) presented a review of studies done on in-field rainwater harvesting with plastic mulch-covered furrow planting of wheat and maize (furrows $1 / 2$ width of the ridge). On average, $10 \%$ more ET was recorded in this system compared to bare soil, flat planting, and with $\mathrm{Y}_{\mathrm{a}}$ comparable to irrigated crops. Most of the ET under furrow plastic mulching was attributed to $\mathrm{T}_{\mathrm{c}}$. Mavimbela and Van Rensburg (2012) investigated the width of runoff water harvesting strips between rows of maize under dryland conditions and irrigated with supplementary and full irrigation. Reduction in $\mathrm{E}_{\mathrm{s}}$ and corresponding increase in $\mathrm{T}_{\mathrm{c}}$ was observed with narrower runoff strips. Evaporation and transpiration were split based on $f_{c}$ (Allen et al., 1998). Full irrigation and $1 \mathrm{~m}$ wide runoff strips produced the highest $\mathrm{Y}_{\mathrm{a}}$ and WP. Three studies were conducted on maize grown in furrow-ridges with plastic mulch in China (Feng et al., 2017; Gong et al. 2017a; and 2017b). They made use of micro-lysimeters and eddy covariance systems to measure the energy balance. Reported reductions in $E_{s}$ ranged from 11.2 to $37 \%$ for maize grown in furrow-ridges with plastic mulching compared to flat field with no mulch.

\subsection{Soil additives and conditioners}

Soil additives and conditioners represent old agricultural practices that improve soil physical properties and fertility (e.g. organic manure). More recently, new materials have been introduced into practice. Biochar is a soil conditioner derived from organic material by pyrolysis. The properties of biochar are variable depending on the parent material and manufacturing process. Addition of biochar to the soil increases porosity, soil water and nutrient retention capacity, soil structure and stability of aggregates (Andrenelli et al., 2016); it affects soil microbiology and it may represent a carbon storage sink, although these interactions remain generally unexplored (Sohi et al., 2010). Similar effects were reported for soil amendments such as humic substances and 
Table 6

Improvements in micro-climate regulation that may reduce non-beneficial water consumption and increase consumptive use water productivity (WP $\mathrm{E}_{\mathrm{ET}}$ ).

\begin{tabular}{|c|c|c|c|}
\hline \multicolumn{2}{|l|}{ Water-saving practices and benefits } & \multicolumn{2}{|l|}{ Potential impacts on actual ET and $\mathrm{WP}_{\mathrm{ET}}$} \\
\hline Water-saving practices & $\begin{array}{l}\text { Commonly recognized main } \\
\text { benefits }\end{array}$ & $\begin{array}{l}\text { Potential impacts on non-beneficial water } \\
\text { consumption and } \mathrm{WP}_{\mathrm{ET}}\end{array}$ & $\begin{array}{l}\text { Potential impacts on actual } \mathrm{K}_{\mathrm{c}} \text { and actual } \\
\mathrm{ET}_{\mathrm{c}}\end{array}$ \\
\hline $\begin{array}{l}\text { Sheltered cultivation }{ }^{1} \\
\quad \text { (depending on materials and openings) }\end{array}$ & $\begin{array}{l}\text { Controlled wind effects } \\
\text { Controlled air humidity } \\
\text { Controlled higher temperature } \\
\text { Lower atmospheric evaporative } \\
\text { demand }\end{array}$ & $\begin{array}{l}\text { Potentially controlled } \mathrm{E}_{\mathrm{s}} \\
\text { Yield increases despite solar energy is reduced } \\
\text { Higher } \mathrm{WP}_{\mathrm{ET}}\end{array}$ & $\begin{array}{l}\text { Reduced } \mathrm{K}_{\mathrm{e}} \\
\mathrm{K}_{\mathrm{cb} \text { act }} \text { high but often smaller than open } \\
\text { field standard } \mathrm{K}_{\mathrm{cb}} \\
\mathrm{K}_{\mathrm{c} \text { act }} \text { and } \mathrm{ET}_{\mathrm{c}} \text { act likely smaller than in } \\
\text { open field }\end{array}$ \\
\hline Wind shields and wind breaks ${ }^{2}$ & $\begin{array}{l}\text { Reduced wind speed } \\
\text { Slightly increased air humidity } \\
\text { Lower atmospheric evaporative } \\
\text { demand }\end{array}$ & $\begin{array}{l}\text { Reduced } \mathrm{E}_{\mathrm{s}} \\
\text { Increased yields } \\
\text { Higher } \mathrm{WP}_{\mathrm{ET}}\end{array}$ & $\begin{array}{l}\text { Reduced } \mathrm{K}_{\mathrm{e}} \\
\text { Increased } \mathrm{K}_{\mathrm{cb} \text { act }} \\
\mathrm{K}_{\mathrm{c} \text { act }} \text { and } \mathrm{ET}_{\mathrm{c} \text { act }} \text { possibly smaller than in } \\
\text { open field }\end{array}$ \\
\hline $\begin{array}{l}\text { Increased } \mathrm{CO}_{2} \text { concentration }{ }^{3} \text { and water vapour } \\
\quad \text { concentrations }\end{array}$ & $\begin{array}{l}\text { Increased water use efficiency } \\
\text { Increased energy use efficiency } \\
\text { Reduced crop water } \\
\text { requirements }\end{array}$ & $\begin{array}{l}\text { Potentially reduced } \mathrm{E}_{\mathrm{s}} \\
\text { Potentially higher yields } \\
\text { Higher } \mathrm{WP}_{\mathrm{ET}}\end{array}$ & $\begin{array}{l}\text { Slightly reduced } \mathrm{K}_{\mathrm{e}} \\
\text { Potentially higher } \mathrm{K}_{\mathrm{cb}} \text { act } \\
\text { Potentially higher } \mathrm{K}_{\mathrm{c} \text { act }} \text { and } \mathrm{ET}_{\mathrm{c} \text { act }}\end{array}$ \\
\hline Micro-climate regulation through irrigation ${ }^{5}$ & $\begin{array}{l}\text { Increased air humidity } \\
\text { Reduced air temperature and } \\
\text { heat shock } \\
\text { Mitigated cold and frost effects }\end{array}$ & $\begin{array}{l}\text { Potentially increased } \mathrm{E}_{\mathrm{s}} \\
\text { Increased yields } \\
\text { Uncertain } \mathrm{WP}_{\mathrm{ET}}\end{array}$ & $\begin{array}{l}\text { Slightly increased } \mathrm{K}_{\mathrm{e}} \\
\text { Potentially higher } \mathrm{K}_{\mathrm{cb}} \text { act } \\
\text { Potentially higher } \mathrm{K}_{\mathrm{c}} \text { and } \mathrm{ET}_{\mathrm{c}}\end{array}$ \\
\hline
\end{tabular}

\footnotetext{
1 Stanghellini et al. (2014); Tanny (2013).

2 Davis and Norman (1988); Campi et al. (2008); Lasco et al. (2014).

3 Vanuytrecht et al. (2012).

4 Zhang et al. (2018).

5 Lobell et al. (2008); Snyder and de Melo-Abreu (2005).
}

compost (Rose et al., 2014; Ramos, 2017), gypsum, organic waste material, organic polymers (Graber et al., 2006; Tadayonnejad et al., 2017), coal fly ash (Shaheen et al., 2014) and zeolites (Hazrati et al., 2017). Physical methods to increase water retention include superabsorbent hydrogels (Guilherme et al., 2015) that have high swelling capacity to absorb water and nutrients for slow release. Soil additives and conditioners may have contradictory impacts on $\mathrm{K}_{\mathrm{c} \text { act }}$ and $\mathrm{K}_{\mathrm{cb}}$ act depending upon the type of crop, the cultivation practices and the climate, however published experiments usually focused on improvements in soil water storage and nutritional properties rather than the effects on crop coefficients.

\section{Micro-climate regulation}

Different practices to condition micro-climate can be employed in order to reduce water losses and improve beneficial water consumption through $\mathrm{T}_{\mathrm{c}}$. Manipulation of micro-climatic variables such as solar radiation, temperature, wind, humidity and $\mathrm{CO}_{2}$ concentration could result in a modified micro-climate and improved conditions for crop growth. This may affect crop development, evapotranspiration and growth, and improve crop water use efficiency (Table 6).

\subsection{Sheltered cultivation}

Sheltered cultivation serves to protect crops from radiation, extreme weather (e.g. wind, hail), pests as well as to regulate micro-climate. The micro-climate under sheltered conditions is very complex and different designs and materials add to the complexity (e.g. open-wall screen, greenhouses, small tunnels, screen material, mesh, color etc.). Differences to open air are enormous in terms of the radiation balance, air temperature, relative humidity and wind (Stanghellini, 2014). Crop coefficients are therefore modified by these conditions, generally with $\mathrm{K}_{\mathrm{c} \text { act }}$ smaller than the standard $\mathrm{K}_{\mathrm{c}}$. Tanny (2013) reviewed the effects of radiation, air velocity, ventilation and turbulence, temperature and humidity on ET and water use efficiency in screen-houses. The outcome was a tendency to reduce $\mathrm{K}_{\mathrm{c}}$ act.

\subsection{Windbreaks and wind shields}

Windbreaks such as trees may mitigate extreme winds that cause soil erosion, sand-blasting, crop mechanical damage and lodging, and they may affect micro-climatic variables such as radiation (shading), temperature, humidity, heat and evaporation fluxes resulting in changes in water and nutrient fluxes. Trees in agroforestry systems exhibit similar effects as windbreaks (Lasco et al., 2014). The effects of tree windbreaks depend on surface roughness, windbreak height, orientation and porosity. Warmer, more humid and less windy conditions usually occur in the sheltered areas. Lower wind speed and more humid conditions reduce vapor pressure deficit $\left(\mathrm{e}_{\mathrm{s}}-\mathrm{e}_{\mathrm{a}}\right)$ and crop ET, mostly without decreasing dry matter assimilation (Tanner and Sinclair, 1983). Reduced $\mathrm{CO}_{2}$ concentrations in the vicinity of the shelter could reduce dry matter assimilation. Reduction of sensible heat fluxes and surface resistances, on the other hand, would most likely favor dry matter assimilation (Davis and Norman, 1988). Higher $Y_{a}$ can therefore be expected in sheltered areas under certain conditions, except when crops compete for resources with the windbreak plants (e.g. shaded areas, competition for water and nutrients by the root systems etc.).

Campi et al. (2008) observed that the effects of windbreaks on $\mathrm{K}_{\mathrm{c}}$ varied with the distance from it and estimated $\mathrm{K}_{\mathrm{c}}$ act by using the FAO56 equation for climate adjustment of $\mathrm{K}_{\mathrm{c}}$ with simulated air humidity and wind speed. No other known articles reported on approaches to modify $\mathrm{K}_{\mathrm{c}}$ due to windbreaks. Using the climate adjustment equation to decrease $K_{c}$ act due to the effect of windbreaks, it is also likely that both $K_{c}$ components decrease, probably $\mathrm{K}_{\mathrm{cb}}$ act less than $\mathrm{K}_{\mathrm{e}}$ (Table 6).

\section{3. $\mathrm{CO}_{2}$ and water vapor concentrations}

The most comprehensive recent publication on the effects of increased $\mathrm{CO}_{2}$ concentrations was a review by Vanuytrecht et al. (2012). They found that $\mathrm{Y}_{\mathrm{a}}$ increased in particular for C3 crops, ET decreased (by reducing stomatal conductance and $\mathrm{T}_{\mathrm{c}}$ ) and WP increased under elevated $\mathrm{CO}_{2}$ concentrations compared to ambient concentrations (Table 6). C3 crops shifted the succession of phenological stages earlier, whilst C4 crops later; the root:shoot ratio generally increased. However, more research was recommended on the interactive effects of $\mathrm{CO}_{2}$ concentrations and other environmental variables on plants. 
Zhang et al. (2018) hypothesized that $\mathrm{e}_{\mathrm{s}}-\mathrm{e}_{\mathrm{a}}$ regulation can reduce irrigation demand by reducing the evaporative demand. This improves plant water status and sustains stomatal function for carbon assimilation. They found that humidification in a greenhouse to maintain moderate $e_{s}-e_{a}$ with fog sprayers contributed to increased $Y_{a}$ of two tomato cultivars and to reduced irrigation requirements.

Under field conditions, spray irrigation may be used to control heat waves (reduce air temperature and heat shocks; Lobell et al., 2008) and to mitigate cold and frost effects (Snyder and de Melo-Abreu, 2005). However, these are expensive and not strictly-speaking water-saving practices. In general, the processes under micro-climatic regulation need to employ complex data collection and modelling of the microclimate, and further research is required on these techniques.

\section{Main outcomes and conclusions}

Two general considerations emanated from this review. Firstly, there is a need to streamline data collection internationally, however following well-defined scientific approaches, and secondly there is a need for optimization of strategies, methods and technologies to reduce non-beneficial water consumption on farms. A more focused collaboration between researchers and equipment manufacturers may help developing guidance issues for the irrigation practiced.

The literature review indicated that published field experiments and modelling work that used the FAO56 approach are generally performed with diverse objectives and not focused on impacts of the diverse techniques and practices on crop ET and/or on crop coefficients, nor on non-beneficial water consumption, thus not allowing an adequate perception of the procedures to modify the crop coefficients for specific conditions. These modifications, as analyzed in papers devoted to updating crop coefficients (Pereira et al., 2020a to d), should be performed through well-focused soil water balance based upon accurate field observations. The importance of measuring all components of the soil-plant-atmosphere continuum is stressed in order to generate complete data sets on the atmospheric and soil environment as well as the plant, which allows appropriate computation of the soil water balance. This includes weather data to calculate $\mathrm{ET}_{\mathrm{o}}$, observations of crop growth and development, assessment of on-farm irrigation system characteristics and performance, measurement of irrigation water volumes and estimation of $\mathrm{K}_{\mathrm{c} \text { act }}$ and $\mathrm{K}_{\mathrm{cb} \text { act }}$ (ideally separating $\mathrm{E}_{\mathrm{s}}$ and $\mathrm{T}_{\mathrm{c}}$ ) in order to estimate irrigation needs and crop $\mathrm{WP}_{\mathrm{ET}}$. This would greatly facilitate the quantification of actual water savings when using welldefined practices that are under-researched (e.g. conservation agriculture, soil amendments, plant conditioners, micro-climate regulation). Based on published experimental findings, on-farm application of the described practices can achieve water savings to a limited extent, usually not exceeding $20 \%$, when compared to sound conventional practices; however, water savings could translate into large volumes at catchment scale.

There is generally a lack of research done on generalizing $\mathrm{K}_{\mathrm{c}}$ coefficients to extrapolate them to different, non-standard conditions. Research is paying little attention to the transferability of crop coefficients, but it should be oriented towards finding the mechanisms of extrapolating and adjusting $\mathrm{K}_{\mathrm{c}}$ and $\mathrm{K}_{\mathrm{cb}}$ for different conditions, including by developing sound empirical or physically-based models, so that there is no need to repeat experimental work for each crop, field and season. Remote sensed vegetation indices may play a very important role, namely when using $\mathrm{f}_{\mathrm{c}}$ or LAI and crop height data to estimate $\mathrm{K}_{\mathrm{cb}}$ and $\mathrm{K}_{\mathrm{c}}$ coefficients with appropriate accuracy (Pereira et al., 2020c, d). Remote sensing also provides for the estimation and mapping of crop coefficients over large areas.

For the application of some water-saving practices, optimization algorithms are required because they involve a large number of possible combinations (e.g. wetted area in micro-irrigation, row spacing and orientation, plant density, application of different types of mulching, infield water harvesting etc.). Optimization is also required when strategies such as deficit irrigation are employed in order to balance water use, yields, $\mathrm{WP}_{\mathrm{ET}}$ and the economics of production considering infrastructural and irrigation system requirements. Experimental work should serve the purpose of calibrating and validating models so that researchers can advise farmers and practitioners on the adoption of optimal water-saving practices at planning, design and application stage. Other practices, such as the use of some plant conditioners, showed promising results in terms of water-savings; however, more field-based research is required for conclusive findings.

Water savings are achievable especially during periods when $f_{c}$ is low and the crops are less sensitive to water stress (RDI). In some instances, substantial reduction of $E_{s}$ from the wetted portion of the ground, even exceeding $20 \%$, can be achieved with some techniques such as mulching and no-till direct planting. However, well-focused research on energy and ET partition is required. Studies that claim large water savings with large increases in yield and $\mathrm{WP}_{\mathrm{ET}}$ should be treated with much caution because such results may defy the physical and physiological boundaries of crop water responses. Such outcomes may originate from poorly designed experiments or poorly managed reference (control) treatments.

Finally, the paper reviewed a wide spectrum of water-saving technologies and practices that have been tried and tested, and that can provide an innovation basket for practitioners farming under different environmental conditions. Large scale implementation of these watersaving practices can benefit the needs for restricting water use and for controlling non-beneficial water consumption in view of water scarcity caused by increased frequency and incidence of drought and climate change effects in many regions of the world, as well as due to the increased competition for water by the non-agricultural sectors. The need for improved water governance with participation of users is essential in view of controlling non-beneficial uses and improving sustainable and economical water use in agriculture.

\section{Declaration of Competing Interest}

The authors declare that they have no known competing financial interests or personal relationships that could have appeared to influence the work reported in this paper.

\section{Acknowledgments}

The support of the Fundação para a Ciência e a Tecnologia (FCT), Portugal granted to the research unit LEAF (UID/AGR/04129/2013) and to the third author (DL 57/2016/CP1382/CT0022) is acknowledged. Isabel Pôças acknowledges the Post-Doctoral grant supported by the Research Infrastructure Enabling Green E-science for the SKA (ENGAGE SKA), reference POCI-01-0145-FEDER-022217, funded by COMPETE 2020 and FCT, Portugal. This research did not receive other specific grants from funding agencies in the public, commercial, or notfor-profit sectors.

\section{Appendix A. Supplementary data}

Supplementary material related to this article can be found, in the online version, at doi:10.1016/j.agwat.2020.106267.

\section{References}

AbdAllah, A.M., Burkey, K.O., Mashaheet, A.M., 2018. Reduction of plant water consumption through anti-transpirants foliar application in tomato plants (Solanum lycopersicum L.). Sci. Hortic. 235, 373-381.

AbdAllah, A.M., Mashaheet, A.M., Zobel, R., Burkey, K.O., 2019. Physiological basis for controlling water consumption by two snap beans genotypes using different antitranspirants. Agric. Water Manage. 214, 17-27.

Adhikari, R., Bristow, K.L., Casey, P.S., Freischmidt, G., Hornbuckle, J.W., Adhikari, B., 2016. Preformed and sprayable polymeric mulch film to improve agricultural water use efficiency. Agric. Water Manage. 169, 1-13.

Adu, M.O., Yawson, D.O., Armah, F.A., Asare, P.A., Frimpong, K.A., 2018. Meta-analysis 
of crop yields of full, deficit, and partial root-zone drying irrigation. Agric. Water Manage. 197, 79-90.

Alberto, M.C.R., Quilty, J.R., Buresh, R.J., Wassmann, R., Haidar, S., Correa Jr., T.Q., Sandro, J.M., 2014. Actual evapotranspiration and dual crop coefficients for dryseeded rice and hybrid maize grown with overhead sprinkler irrigation. Agric. Water Manage. 136, 1-12.

Alberto, M.C.R., Wassmann, R., Hirano, T., Miyata, Hatano R., Kumar, A., Padre, A., Amante, M., 2011. Comparisons of energy balance and evapotranspiration between flooded and aerobic rice fields in the Philippines. Agric. Water Manage. 98, 1417-1430.

Allen, R.G., Pereira, L.S., 2009. Estimating crop coefficients from fraction of ground cover and height. Irrig. Sci. 28, 17-34. https://doi.org/10.1007/s00271-009-0182-z.

Allen, R.G., Pereira, L.S., Howell, T.A., Jensen, M.E., 2011. Evapotranspiration information reporting: I. Factors governing measurement accuracy. Agric. Water Manage. 98 (6), 899-920. https://doi.org/10.1016/j.agwat.2010.12.015.

Allen, R.G., Pereira, L.S., Raes, D., Smith, M., 1998. Crop evapotranspiration: Guidelines for computing crop water requirements. FAO Irrigation and Drainage Bulletin No 56. FAO, Rome, Italy $300 \mathrm{pp}$.

Allen, R.G., Pereira, L.S., Smith, M., Raes, D., Wright, J., 2005a. FAO-56 Dual crop coefficient method for estimating evaporation from soil and application extensions. J. Irrig. Drain. E-ASCE 131, 2-13.

Allen, R.G., Pruitt, W.O., Raes, D., Smith, M., Pereira, L.S., 2005b. Estimating evaporation from bare soil and the crop coefficient for the initial period using common soils information. J. Irrig. Drain. E-ASCE 131 (1), 14-23. https://doi.org/10.1061/(ASCE) 0733-9437(2005)131:1(14).

Anderson, M.C., Allen, R.G., Morse, A., Kustas, W.P., 2012. Use of Landsat thermal imagery in monitoring evapotranspiration and managing water resources. Remote Sens. Environ. 122, 50-65. https://doi.org/10.1016/j.rse.2011.08.025.

Andrenelli, M.C., Maienza, A., Genesio, L., Miglietta, F., Pellegrini, S., Vaccari, F.P., Vignozzi, N., 2016. Field application of pelletized biochar: Short term effect on the hydrological properties of a silty clay loam soil. Agric. Water Manage. 163, 190-196.

Arif, C., Setiawan, B.I., Sofiyuddin, H.A., Martief, L.M., Mizoguchi, M., Doi, R., 2012. Estimating crop coefficient in an intermittent irrigation paddy field using Excel Solver. Rice Sci. 19 (2). https://doi.org/10.1016/S1672-6308(12)60033-X.

Arnold, B.J., Upadhyaya, S.K., Wallender, W.W., Grismer, M.E., 2015. Sensor-based cutoff strategy for border check-irrigated fields. J. Irrig. Drain. Eng. 141 (7), 04014081.

Astacio, M.G., van Iersel, M.W., 2011. Determining the effects of abscisic acid drenches on evapotranspiration and leaf gas exchange of tomato. HortSci. 46 (11), 1512-1517.

Bai, M., Xu, D., Li, Y., Zhang, S., Liu, S., 2017. Coupled impact of spatial variability of infiltration and microtopography on basin irrigation performances. Irrig. Sci. 35, 437-449.

Bai, M.J., Xu, D., Li, Y.N., Pereira, L.S., 2011. Impacts of spatial variability of basins microtopography on irrigation performance. Irrig. Sci. 29, 359-368.

Balwinder-Singh Eberbacha, P.L., Humphreys, E., Kukal, S.S., 2011. The effect of rice straw mulch on evapotranspiration, transpiration and soil evaporation of irrigated wheat in Punjab. India. Agric. Water Manage. 98, 1847-1855.

Bittelli, M., Flury, M., Campbell, S., Nichols, E., 2001. Reduction of transpiration through foliar application of chitosan. Agric. Forest Meteorol. 107, 167-175.

Boari, F., Cantore, V., Di Venere, D., Sergio, L., Candido, V., Schiattone, M.I., 2019. Pyraclostrobin can mitigate salinity stress in tomato crop. Agric. Water Manage. 222, 254-264.

Boari, F., Donadio, A., Schiattone, M.I., Cantore, V., 2015. Particle film technology: a supplemental tool to save water. Agric. Water Manage. 147, 154-162.

Bodner, G., Nakhforoosh, A., Kaul, H.-P., 2015. Management of crop water under drought: a review. Agron. Sustain. Dev. 35, 401-442. https://doi.org/10.1007/ s13593-015-0283-4.

Bouma, J.A., Hegde, S.S., Lasage, R., 2016. Assessing the returns to water harvesting: A meta-analysis. Agric. Water Manage. 163, 100-109.

Brillante, L., Belfiore, N., Gaiotti, F., Lovat, L., Sansone, L., Poni, S., Tomasi, D., 2016. Comparing Kaolin and Pinolene to improve sustainable grapevine production during drought. PLOS ONE 11 (6), e0156631.

Bush, A., Elamin, A.M., Ali, A.B., Hong, L., 2016. Effect of different operating pressures on the hydraulic performance of drip irrigation system in Khartoum State conditions. J. Environ. Agric Sci. 6, 64-68.

Cai, J., Liu, Y., Xu, D., Paredes, P., Pereira, L.S., 2009. Simulation of the soil water balance of wheat using daily weather forecast messages to estimate the reference evapotranspiration. Hydrol. Earth Syst. Sci. 13, 1045-1059.

Calera, A., Campos, I., Osann, A., D’Urso, G., Menenti, M., 2017. Remote sensing for crop water management: from ET modelling to services for the end users. Sensors 17 (5), 1104. https://doi.org/10.3390/s17051104.

Campi, P., Palumbo, A.D., Mastrorilli, M., 2008. The evapotranspiration of crop protected by windbreak. In: In: Santini, A., Lamaddalena, N., Severino, G., Palladino, M. (Eds.), Irrigation in Mediterranean Agriculture: Challenges and Innovation for the Next Decades 84. CIHEAM, Bari, pp. 135-141 Options Méditerranéennes: Série A. Séminaires Méditerranéens.

Campos, I., Neale, C.M.U., Arkebauer, T.J., Suyker, A.E., Gonçalves, I.Z., 2018. Water productivity and crop yield: A simplified remote sensing driven operational approach. Agric. For. Meteorol. 249, 501-511. https://doi.org/10.1016/j.agrformet. 2017.07.018.

Campos, I., Neale, C.M.U., Suyker, A.E., Arkebauer, T.J., Gonçalves, I.Z., 2017. Reflectance-based crop coefficients REDUX: For operational evapotranspiration estimates in the age of high producing hybrid varieties. Agric. Water Manage. 187, $140-153$.

Canellas, L.P., Olivares, F.L., Aguiar, N.A., Jones, D.L., Nebbioso, A., Mazzei, P., Piccolo, A., 2015. Humic and fulvic acids as biostimulants in horticulture. Sci. Hortic. 196, $15-27$.
Cantore, V., Lechkar, O., Karabulut, E., Sellami, M.H., Albrizio, R., Boari, F., Stellacci, A.M., Todorovic, M., 2016. Combined effect of deficit irrigation and strobilurin application on yield, fruit quality and water use efficiency of "cherry" tomato (Solanum lycopersicum L.). Agric. Water Manage. 167, 53-61.

Cantore, V., Pace, B., Albrizio, R., 2009. Effects of kaolin-based particle film technology on tomato physiology, yield and quality. Environ. Exp. Bot. 66, 279-288.

Capra, A., Consoli, S., Scicolone, B., 2008. Deficit irrigation: Theory and practice. In: Alonso, D., Iglesias, H.J. (Eds.), Agricultural Irrigation Research Progress. Nova Science Publishers, Inc., New York, USA, pp. 53-82.

Cavero, J., Faci, J.M., Martínez-Cob, A., 2016. Relevance of sprinkler irrigation time of the day on alfalfa forage production. Agric. Water Manage. 178, 304-313.

Cesari de Maria, S., Rienzner, M., Facchi, A., Chiaradia, E.A., Romani, M., Gandolfi, C., 2016. Water balance implications of switching from continuous submergence to flush irrigation in a rice-growing district. Agric. Water Manage. 171, 108-119.

Chauhdary, J.N., Bakhsh, A., Ahmad, N., Mehmood, K., 2015. Optimizing chlorine use for improving performance of drip irrigation system under biologically contaminated water source. Pak. J. Agri. Sci. 52 (3), 829-835.

Chen, T.H.H., Murata, N., 2011. Glycinebetaine protects plants against abiotic stress: mechanisms and biotechnological applications. Plant Cell Environ. 34, 1-20.

Cohen, I., Netzer, Y., Sthein, I., Gilichinsky, M., Tel-Or, E., 2019. Plant growth regulators improve drought tolerance, reduce growth and evapotranspiration in deficit irrigated Zoysia japonica under field conditions. Plant Growth Regul. 88, 9-17. https://doi.org/ 10.1007/s10725-019-00484-4.

Colaizzi, P.D., Schneider, A.D., Evett, S.R., Howell, T.A., 2004. Comparison of SDI, LEPA, and spray irrigation performance for grain sorghum. Trans. ASAE 47 (5), 1477-1492.

Colla, G., Nardi, S., Cardarelli, M., Ertani, A., Lucini, L., Canaguier, R., Rouphael, Y., 2015. Protein hydrolysates as biostimulants in horticulture. Sci. Hortic. 196, 28-38.

Cosic, M., Djurovic, N., Todorovic, M., Maletic, R., Zecevic, B., Stricevic, R., 2015. Effect of irrigation regime and application of kaolin on yield, quality and water use efficiency of sweet pepper. Agric. Water. Manage. 159, 139-147.

D’Urso, G., Richter, K., Calera, A., Osann, M.A., Escadafal, R., Garatuza-Payan, J., Hanich, L., Perdigão, A., Tapia, J.B., Vuolo, F., 2010. Earth Observation products for operational irrigation management in the context of the PLEIADeS project. Agric. Water Manage. 98, 271-282.

Darouich, H., Cameira, M.R., Gonçalves, J.M., Paredes, P., Pereira, L.S., 2017. Comparing sprinkler and surface irrigation for wheat using multi-criteria analysis: water saving vs. economic returns. Water 9, 50. https://doi.org/10.3390/w9010050.

Darouich, H., Gonçalves, J.M., Muga, A., Pereira, L.S., 2012. Water saving vs. farm economics in cotton surface irrigation: An application of multicriteria analysis. Agric. Water Manage. 115, 223-231.

Darouich, H., Pedras, C.M.G., Gonçalves, J.M., Pereira, L.S., 2014. Drip vs. surface irrigation: a comparison focusing on water saving and economic returns using multicriteria analysis applied to cotton. Biosyst. Eng. 122, 74-90. https://doi.org/10. 1016/j.biosystemseng.2014.03.010.

Daryanto, S., Wan, L., Jacinthe, P.-A., 2017. Can ridge-furrow plastic mulching replace irrigation in dryland wheat and maize cropping systems? Agric. Water Manage. 190, 1-5.

Davenport, D.C., Fisher, M.A., Hagan, R.M., 1972. Some counteractive effects of antitranspirants. Plant Physiol. 49, 722-724.

Davis, J.E., Norman, J.M., 1988. Effects of shelter on plant water use. Agric. Ecosyst. Environ. 393-402 22/23.

Dechmi, F., Playan, E., Cavero, J., Faci, J.M., Martinez-Cob, A., 2003. Wind effects on solid set sprinkler irrigation depth and yield of maize (Zea mays). Irrig. Sci. 19, $165-173$.

DeLaune, P.B., Mubvumba, P., Ale, S., Kimura, E., 2020. Impact of no-till, cover crop, and irrigation on cotton yield. Agric. Water Manage. 232, 106038.

Ding, R., Kang, S., Zhang, Y., Hao, X., Tong, L., Du, T., 2013. Partitioning evapotranspiration into soil evaporation and transpiration using a modified dual crop coefficient model in irrigated maize field with ground-mulching. Agric. Water Manage. 127, 85-96.

Doorenbos, J., Kassam, A.H., 1979. Yield Response to Water. Irrig. Drain. Paper 33. FAO, Rome, pp. 193.

Doorenbos, J., Pruitt, W.O., 1977. Crop Water Requirements. Irrig. Drain. Paper 24. FAO, Rome, pp. 144.

Du Jardin, P., 2015. Plant biostimulants: definition, concept, main categories and regulation. Sci Hortic. 30 (196), 3-14.

Du, T., Kang, S., Yan, B., Zhang, J., 2013. Alternate furrow irrigation: A practical way to improve grape quality and water use efficiency in arid Northwest China. J. Integr. Agric. 12 (3), 509-519.

Dukes, M.D., 2006. Effect of wind speed and pressure on linear move irrigation system uniformity. Appl. Eng. Agric. 22 (4), 541-548.

El Mantawy, R.F., El Bialy, M., 2018. Effect of antitranspirants application on growth and productivity of sunflower under soil moisture stress. Nature and Science 16 (2), 92-106.

Enciso-Medina, J., Multer, W.L., Lamm, F.R., 2011. Management, maintenance, and water quality effects on the long-term performance of subsurface drip irrigation systems. Appl. Eng. Agric. 27 (6), 969-978.

Fan, Y., Din, R., Kang, S., Hao, X., Du, T., Tong, L., Li, S., 2017. Plastic mulch decreases available energy and evapotranspiration and improves yield and water use efficiency in an irrigated maize cropland. Agric. Water Manage. 179, 122-131.

Fandiño, M., Cancela, J.J., Rey, B.J., Martínez, E.M., Rosa, R.G., Pereira, L.S., 2012. Using the dual-Kc approach to model evapotranspiration of albariño vineyards (Vitis vinifera L. $c v$. albariño) with consideration of active ground cover. Agric. Water Manage 112, 75-87.

Fangmeier, D.D., Clemmens, A.J., El-Ansary, M., Strelkoff, T.S., Osman, H.E., 1999. Influence of land leveling precision on level-basin advance and performance. Trans 
ASAE 42 (4), 1019-1025.

FAO, 2016. AQUASTAT Main Database, Food and Agriculture Organization of the United Nations (FAO). (accessed 17 April 2020. http://www.fao.org/nr/water/aquastat/ data/query/index.html? lang $=$ en.

Farahani, H.J., Gabriella, I., Oweis, T.Y., 2009. Parameterization and evaluation of the AquaCrop model for full and deficit irrigated cotton. Agron. J. 101, 469-476.

Faralli, M., Grove, I.G., Hare, M.C., Alcalde-Barrios, A., Williams, K.S., Corke, F.M.K., Kettlewell, P.S., 2017. Modulation of Brassica napus source-sink physiology through film antitranspirant induced drought tolerance amelioration that is dependent on the stress magnitude. J. Agro. Crop Sci. 203, 360-372.

Feng, Y., Gong, D., Mei, X., Hao, W., Tang, D., Cui, N., 2017. Energy balance and partitioning in partial plastic mulched and non-mulched maize fields on the Loess Plateau of China. Agric. Water Manage. 191, 193-206.

Feng, Y., Hao, W., Gao, L., Li, H., Gong, D., Cui, N., 2019. Comparison of maize water consumption at different scales between mulched and non-mulched croplands. Agric. Water Manage. 216, 315-324.

Fereres, E., Soriano, M.A., 2007. Deficit irrigation for reducing agricultural water use. J. Exp. Bot. 58 (2), 147-159.

Galindo, A., Collado-González, J., Grinán, I., Corell, M., Centeno, A., Martín-Palomo, M.J., Girón, I.F., Rodríguez, P., Cruz, Z.N., Memmi, H., Carbonell-Barrachina, A.A., Hernández, F., Torrecillas, A., Moriana, A., López-Pérez, D., 2018. Deficit irrigation and emerging fruit crops as a strategy to save water in Mediterranean semiarid agrosystems. Agric. Water Manage. 202, 311-324.

Giannakoula, A.E., Ilias, I.F., Maksimović, J.J.D., Maksimović, V.M., Živanović, B.D., 2012. The effects of plant growth regulators on growth, yield, and phenolic profile of lentil plants. J. Food Comp. Anal. 28, 46-53.

Giménez, L., Paredes, P., Pereira, L.S., 2017. Water use and yield of soybean under various irrigation regimes and severe water stress. Application of AquaCrop and SIMDualKc models. Water 9, 393. https://doi.org/10.3390/w9060393.

Gonçalves, J.M., Muga, A.P., Horst, M.G., Pereira, L.S., 2011. Furrow irrigation design with multicriteria analysis. Biosyst. Eng. 109, 266-275.

Gong, D., Mei, X., Hao, W., Wang, H., Caylor, K.K., 2017a. Comparison of ET partitioning and crop coefficients between partial plastic mulched and non-mulched maize fields. Agric. Water Manage. 181, 23-34.

Gong, D., Mei, X., Hao, W., Wang, H., Caylor, K.K., 2017b. Comparison of multi-level water use efficiency between plastic film partially mulched and non-mulched croplands at eastern Loess Plateau of China. Agric. Water Manage. 179, 215-226.

González-Dugo, M.P., Neale, C.M.U., Mateos, L., Kustas, W.P., Prueger, J.H., Anderson, M.C., Li, F., 2009. A comparison of operational remote sensing-based models for estimating crop evapotranspiration. Agric. Forest Meteorol. 149, 1843-1853.

Graber, E.R., Fine, P., Levy, G.J., 2006. Soil stabilization in semiarid and arid land agriculture. J. Mater. Civil Engn. 18 (2), 190-205. https://doi.org/10.1061/ ASCE0899-1561.

Green, R.L., Kim, K.S., Beard, J.B., 1990. Effects of Flurprimidol, Mefluidide, and soil moisture on St. Augustinegrass evapotranspiration rate. HortSci. 25 (4), 439-441.

Grum, B., Assefa, D., Hessel, R., Woldearegay, K., Ritsema, C.J., Aregawi, B., Geissen, V., 2017. Improving on-site water availability by combining in-situ water harvesting techniques in semi-arid Northern Ethiopia. Agric. Water Manage. 193, 153-162.

Guilherme, M.R., Aouada, F.A., Fajardo, A.R., Martins, A.F., Paulino, A.T., Davi, M.F.T., Rubira, A.F., Muniz, E.C., 2015. Superabsorbent hydrogels based on polysaccharides for application in agriculture as soil conditioner and nutrient carrier: A review. Eur. Polym. J. 72, 365-385.

Hazrati, S., Tahmasebi-Sarvestani, Z., Mokhtassi-Bidgoli, A., Modarres-Sanavy, S.A.M., Mohammadi, H., Nicola, S., 2017. Effects of zeolite and water stress on growth, yield and chemical compositions of Aloe vera L. Agric. Water Manage. 181, 66-72.

He, J., Zhang, X., Flerchinger, G., Yu, Q., Ma, L. (Eds.), 2019. Plastic mulching effects on water use efficiency, crop growth, and soil health in Northwestern China. Agric. Water Manage Special Issue.

Hoffman, G.J., Evans, R.G., Jensen, M.E., Martin, D.L., Elliot, R.L. (Eds.), 2007. Design and Operation of Farm Irrigation Systems, 2nd Edition. ASABE, St. Joseph, MI.

Hornbuckle, J., 2014. Vineyard Irrigation-Delivering Water Savings through Emerging Technology. Final Report to Grape and Wine Research \& Development Corporation. CSIRO Land and Water. (accessed 30 April 2020)Canberra, Australia. https://www. wineaustralia.com/getmedia/6e7c3e92-c36c-4685-9bf8-9039d99aa4a0/CSL-0901Final-Report-IrriSAT.

Hornbuckle, J.W., Car, N.J., Christen, E.W., Stein, T.-M., Williamson, B., 2009. IrriSatSMS Irrigation water management by satellite and SMS - A utilisation framework CRC for Irrigation Futures Technical Report No. 01/09. CSIRO Land and Water Science Report No. 04/09. pp. 64.

Horst, M.G., Shamutalov, Sh., Gonçalves, J.M., Pereira, L.S., 2007. Assessing impacts of surge-flow irrigation on water saving and productivity of cotton. Agric. Water Manage. 87 (2), 115-127. https://doi.org/10.1016/j.agwat.2006.06.014.

Hummel, R.L., 1990. Water relations of container-grown woody and herbaceous plants following antitranspirant sprays. HortSci. 25 (7), 772-775.

Humpherys, A.S., 1989. Surge irrigation: 1. An overview. ICID Bull 38 (2), 35-48.

Humpherys, A.S., 1995. Semiautomation of irrigated basins and borders: I. Single-function turnout gates. Appl. Eng. Agric. 11 (1), 67-74.

Hutsch, B.W., Schubert, S., 2017. Maize harvest index and water use efficiency can be improved by inhibition of gibberellin biosynthesis. J. Agro. Crop Sci. 204, 209-218.

Intrigliolo, D.S., Castel, J.R., 2009. Response of Vitis vinifera cv. 'Tempranillo' to partial rootzone drying in the field: Water relations, growth, yield and fruit and wine quality. Agric. Water Manage 96, 282-292.

IPCC, 2014. Climate Change 2014: Synthesis Report. In: Pauchari, R.K., Meyer, L.A. (Eds.), Contribution of Working Groups I, II and III to the Fifth Assessment Report of the Inter-governmental Panel on Climate Change. Core Writing Team. Geneva, Switzerland: IPCC, pp. 151.
Iriti, M., Picchi, V., Rossoni, M., Gomarasca, S., Ludwig, N., Gargano, M., Faoro, F., 2009. Chitosan antitranspirant activity is due to abscisic acid-dependent stomatal closure. Environ. Exper. Bot. 66, 493-500.

Jacques, D., Fox, G., White, P., 2018. Farm level economic analysis of subsurface drip irrigation in Ontario corn production. Agric. Water Manage. 203, 333-343.

Kang, S., Hao, X., Du, T., Tong, L., Su, X., Lu, H., Li, X., Huo, Z., Li, S., Ding, R., 2017. Improving agricultural water productivity to ensure food security in China under changing environment: From research to practice. . Agric. Water Manage. 179, 5-17.

Kassam, A., Derpsch, R., Friedrich, T., 2014. Global achievements in soil and water conservation: The case of Conservation Agriculture. Int. Soil Water Conserv. Res. 2 (1), 5-13.

Keller, J., Bliesner, R.D., 1990. Sprinkle and Trickle Irrigation. Van Nostrand Reinhold. New York, USA.

Kettlewell, P.S., Heath, Haigh I.M., 2010. Yield enhancement of droughted wheat by film antitranspirant application: rationale and evidence. Agric. Sci. 1 (3), 143-147.

Klocke, N.L., Currie, R.S., Aiken, R.M., 2009. Soil water evaporation and crop residues. Trans. ASABE 52 (1), 103-110.

Lamaddalena, N., Frattino, U., Daccache, A., 2007. On-farm Sprinkler Irrigation Performance as affected by the Distribution System. Biosyst. Eng. 96, 99-109.

Lamm, F.R., Ayars, J.E., Nakayama, F.S. (Eds.), 2006. Microirrigation for crop production: Design, operation, and management. Elsevier, The Netherlands.

Lampurlanés, J., Angás, P., Cantero-Martínez, C., 2002. Tillage effects on water storage during fallow, and on barley root growth and yield in two contrasting soils of the semi-arid Segarra region in Spain. Soil Till. Res. 65, 207-220.

Lasco, R.D., Delfino, R.J.P., Espaldon, M.L.O., 2014. Agroforestry systems: helping smallholders adapt to climate risks while mitigating climate change. WIREs Clim Change 5, 825-833. https://doi.org/10.1002/wcc.301.

Li, D., Franssen, H.-J.H., Han, X., Jiménez-Bello, M.A., Alzamora, F.M., Vereecken, H., 2018. Evaluation of an operational real-time irrigation scheduling scheme for drip irrigated citrus fields in Picassent. Spain. Agric. Water Manage. 208, 465-477.

Liang, S., Xu, X., Lu, Z., 2018. Effect of azoxystrobin fungicide on the physiological and biochemical indices and ginsenoside contents of ginseng leaves. J. Ginseng Res. 42, 175-182.

Lobell, D.B., Bonfils, C.J., Kueppers, L.M., Snyder, M.A., 2008. Irrigation cooling effect on temperature and heat index extremes. Geophys. Res. Lett. 35, L09705. https://doi. org/10.1029/2008GL034145.

Lorite, I.J., Ramírez Cuesta, J.M., ·Cruz-Blanco, M., Santos, C., 2015. Using weather forecast data for irrigation scheduling under semi arid conditions. Irrig. Sci. 33 411-427.

Ma, D., Chen, L., Qu, H., Wang, Y., Misselbrook, T., Jiang, R., 2018. Impacts of plastic film mulching on crop yields, soil water, nitrate, and organic carbon in Northwestern China: A meta-analysis. Agric. Water Manage. 202, 166-173.

Manning, D.T., Lurbé, S., Comas, L.H., Trout, T.J., Flynn, N., Fonte, S.J., 2018. Economic viability of deficit irrigation in the Western US. Agric. Water Manage. 196, 114-123.

Mantovani, E.C., Villalobos, F.J., Orgaz, F., Fereres, E., 1995. Modelling the effects of sprinkler irrigation uniformity on crop yield. Agric. Water Manage. 27, 243-257.

Mao, Z., Dong, B., Pereira, L.S., 2004. Assessment and water saving issues for Ningxia paddies, upper Yellow River Basin. Paddy and Water Environ. 2 (2), 99-110.

Martins, J.D., Rodrigues, G.C., Paredes, P., Carlesso, R., Oliveira, Z.B., Knies, A.E., Petry, M.T., Pereira, L.S., 2013. Dual crop coefficients for maize in southern Brazil: Model testing for sprinkler and drip irrigation and mulched soil. Biosyst. Eng. 115, 291-310.

Masseroni, D., Uddin, J., Tyrrell, R., Mareels, I., Gandolfi, C., Facchi, A., 2017. Towards a smart automated surface irrigation management in rice-growing areas in Italy. J. Agric. Eng. 48 (1), 42-48.

Mateos, L., González-Dugo, M.P., Testi, L., Villalobos, F.J., 2013. Monitoring evapotranspiration of irrigated crops using crop coefficients derived from time series of satellite images. I. Method validation. Agric. Water Manage. 125, 81-91.

Mavimbela, S.S.W., Van Rensburg, L.D., 2012. Integrating micro-flood irrigation with infield rainwater harvesting: Maize yield and water use efficiency. Irrig. and Drain. 61 (2), 70-81.

Mazarei, R., Mohammadi, A.S., Naseri, A.A., Ebrahimian, H., Izadpanah, Z., 2020. Optimization of furrow irrigation performance of sugarcane fields based on inflow and geometric parameters using WinSRFR in Southwest of Iran. Agric. Water Manage. 228, 105899.

Melton, F.S., Johnson, L.F., Lund, C.P., Pierce, L.L., Michaelis, A.R., Hiatt, S.H., Guzman, A., Adhikari, D.D., Purdy, A.J., Rosevelt, C., Votava, P., Trout, T.J., Temesgen, B., Frame, K., Sheffner, E.J., Nemani, R.R., 2012. Satellite irrigation management support with the terrestrial observation and prediction system: A framework for integration of satellite and surface observations to support improvements in agricultural water resource management. IEEE Journal of Selected Topics in Applied Earth Observations and Remote Sensing 5, 1709-1721.

Merriam, J.L., Keller, I., 1978. Farm Irrigation System Evaluation: A Guide for Management. Utah State University, Logan, USA.

Miao, Q., Rosa, R.D., Shi, H., Paredes, P., Zhu, L., Dai, J., Gonçalves, J.M., Pereira, L.S., 2016. Modeling water use, transpiration and soil evaporation of spring wheat-maize and spring wheat-sunflower relay intercropping using the dual crop coefficient approach. Agric. Water Manage. 165, 211-229.

Miao, Q., Shi, H., Gonçalves, J.M., Pereira, L.S., 2015. Field assessment of basin irrigation performance and water saving in Hetao, Yellow River basin: issues to support irrigation systems modernization. Biosyst. Eng. 136, 102-116.

Miao, Q., Shi, H., Gonçalves, J., Pereira, L.S., 2018. Basin irrigation design with multicriteria analysis focusing on water saving and economic returns. Application to wheat in Hetao, Yellow River basin. Water 10, 67. https://doi.org/10.3390/w10010067.

Minhas, P.S., Ramos, T.B., Ben-Gal, A., Pereira, L.S., 2020. Coping with salinity in irrigated agriculture: Crop evapotranspiration and water management issues. Agric. Water Manage. 227, 105832. https://doi.org/10.1016/j.agwat.2019.105832. 
Molle, F., Tanouti, O., 2017. Squaring the circle: Agricultural intensification vs. water conservation in Morocco. Agric. Water Manage. 192, 170-179.

Morell, F.J., Lampurlanés, J., Álvaro-Fuentes, J., Cantero-Martinez, C., 2011. Yield and water use efficiency of barley in a semiarid Mediterranean agroecosystem: Long-term effects of tillage and $\mathrm{N}$ fertilization. Soil Till. Res. 117, 76-84.

Morris, M.R., Hussain, A., Gillies, M.H., O'Halloran, N.J., 2015. Inflow rate and border irrigation performance. Agric Water Manage. 155, 76-86.

Mostafa, G.G., Abou Al-Hamd, M.F., 2011. Effect of gibberellic acid and indole-3 acetic acid on improving growth and accumulation of phytochemical composition in Balanites aegyptica plants. Amer. J. Plant Physiol. 6, 36-43.

Niblack, M., Sanchez, C.A., 2008. Automation of surface irrigation by cut-off time or cutoff distance control. Appl. Eng. Agric. 24 (5), 611-614.

Oweis, T.Y., Prinz, D., Hachum, A.Y., 2012. Water Harvesting for Agriculture in the Dry Areas. CRC Press, Boca Raton, FL, USA.

Palliotti, A., Panara, F., Famiani, F., Sabbatini, P., Howell, G.S., Silvestroni, O., Poni, S., 2013. Postveraison application of antitranspirant di-1-p menthene to control sugar accumulation in Sangiovese grapevines. Amer. J. Enol. Vitic. 64, 378-385.

Panigrahi, P., Raychaudhuri, S., Thakur, A.K., Nayak, A.K., Sahu, P., Ambast, S.K., 2019. Automatic drip irrigation scheduling effects on yield and water productivity of banana. Sci. Hortic. 257, 108677.

Paredes, P., D’Agostino, D., Assif, M., Todorovic, M., Pereira, L.S., 2018b. Assessing potato transpiration, yield and water productivity under various water regimes and planting dates using the FAO dual Kc approach. Agric. Water Manage. 195, 11-24.

Paredes, P., Martins, D., Pereira, L.S., Cadima, J., Pires, C., 2018a. Accuracy of daily estimation of grass reference evapotranspiration using ERAInterim reanalysis products with assessment of alternative bias correction schemes. Agric. Water Manage. 210, 340-353.

Paredes, P., Pereira, L.S., Almorox, J., Darouich, H., 2020. Consolidated methods to compute reference grass evapotranspiration with reduced data sets by parameterizing the FAO Penman-Monteith temperature approach and the Hargeaves-Samani equation using regional climatic variables. Agric. Water Manage submitted for publication.

Paredes, P., Ribeiro, A.F.S., Pires, C.A.L., Pereira, L.S., 2015. Usabilidade de previsões sazonais de ensemble da temperatura e precipitação na modelação da rega e da produção. Aplicação a cevada e milho. In: Pires, C.A.L., Pereira, L.S. (Eds.), Predictabilidade Sazonal de Secas. Avaliação ao Nível Regional e Agrícola. ISAPress, Lisbon, Portugal, pp. 307-332 in Portuguese.

Paredes, P., Rodrigues, G.C., Alves, I., Pereira, L.S., 2014. Partitioning evapotranspiration, yield prediction and economic returns of maize under various irrigation management strategies. Agric. Water Manage 135, 27-39. https://doi.org/10.1016/j.agwat.2013. 12.010 .

Paredes, P., Rodrigues, G.C., Cameira, M.R., Torres, M.O., Pereira, L.S., 2017. Assessing yield, water productivity and farm economic returns of malt barley as influenced by the sowing dates and supplemental irrigation. Agric. Water Manage. 179, 132-143.

Pedras, C.M.G., Pereira, L.S., 2009. Multicriteria analysis for design of microirrigation systems. Application and sensitivity analysis. Agric. Water Manage 96, 702-710. https://doi.org/10.1016/j.agwat.2008.10.007.

Pereira, L.S., 1999. Higher performance through combined improvements in irrigation methods and scheduling: a discussion. Agric. Water Manage. 40, 153-169.

Pereira, L.S., 2017. Water, agriculture and food: challenges and issues. Water Resour. Manage. 31, 2985-2999.

Pereira, L.S., Allen, R.G., Smith, M., Raes, D., 2015a. Crop evapotranspiration estimation with FAO56: Past and future. Agric. Water Manage. 147, 4-20.

Pereira, L.S., Cai, L.G., Hann, M.J., 2003. Farm water and soil management for improved water use in the North China Plain. Irrigation and Drainage 52 (4), 299-317.

Pereira, L.S., Cordery, I., Iacovides, I., 2009a. Coping with Water Scarcity: Addressing the Challenges. Springer Science + Business Media B.V. Dordrecht, The Netherlands.

Pereira, L.S., Cordery, I., Iacovides, I., 2012. Improved indicators of water use performance and productivity for sustainable water conservation and saving. Agric. Water Manage. 108, 39-51.

Pereira, L.S., Gonçalves, J.M., 2018. Surface irrigation. Oxford Encyclopedia of Agriculture and the Environment. Oxford University Press, Oxford, UK. https://doi. org/10.1093/acrefore/9780199389414.013.248.

Pereira, L.S., Gonçalves, J.M., Dong, B., Mao, Z., Fang, S.X., 2007. Assessing basin irrigation and scheduling strategies for saving irrigation water and controlling salinity in the Upper Yellow River basin. China. Agric. Water Manage. 93 (3), 109-122.

Pereira, L.S., Oweis, T., Zairi, A., 2002. Irrigation management under water scarcity. Agric. Water Manage. 57, 175-206.

Pereira, L.S., Paredes, P., Hunsaker, D., López-Urrea, R., Shad, Z.M., 2020b. Standard single and basal crop coefficients for field crops. An update and advances of FAO56 crop water requirements method. Agric. Water Manage submitted for publication.

Pereira, L.S., Paredes, P., Cholpankulov, E.D., Inchenkova, O.P., Teodoro, P.R., Horst, M.G., 2009b. Irrigation scheduling strategies for cotton to cope with water scarcity in the Fergana Valley, Central Asia. Agric. Water Manage. 96 (5), 723-735.

Pereira, L.S., Paredes, P., López-Urrea, R., Hunsaker, D., Mota, M., Shad, Z.M., 2020a. Standard single and basal crop coefficients for vegetable crops to update FAO56 crop water requirements method. Agric. Water Manage submitted for publication.

Pereira, L.S., Paredes, P., Melton, F., Wang, T., Johnson, L., López-Urrea, R., Cancela, J., Allen, R., 2020c. Prediction of crop coefficients from fraction of ground cover and height. Background and validation using ground and remote sensing data. Agric. Water Manage submitted for publication.

Pereira, L.S., Paredes, P., Mota, M., Melton, F., Wang, T., 2020d. Basal crop coefficients from fraction of ground cover and height: application to vegetable, field and tree fruit crops. Agric. Water Manage submitted for publication.

Pereira, L.S., Paredes, P., Rodrigues, G.C., Neves, M., 2015b. Modeling malt barley water use and evapotranspiration partitioning in two contrasting rainfall years. Assessing
AquaCrop and SIMDualKc models. Agric. Water Manage. 159, 239-254. https://doi. org/10.1016/j.agwat.2015.06.006.. Corrigendum in Agric. Water Manage. 163, 408. https://doi.org/10.1016/j.agwat.2015.08.017, 2016.

Pereira, L.S., Perrier, A., Allen, R.G., Alves, I., 1999a. Evapotranspiration: Review of concepts and future trends. J. Irrig. Drain. Eng. 125 (2), 45-51. https://doi.org/10. 1061/(ASCE)0733-9437(1999)125:2(45).

Pereira, L.S., Trout, T.J., 1999b. Irrigation Methods. In: van Lier, H.N., Pereira, L.S., Steiner, F.R. (Eds.), CIGR Handbook of Agricultural Engineering, Vol. I: Land and Water Engineering. ASAE, St. Joseph, MI, USA, pp. 297-379.

Perry, C., 2011. Accounting for water use: Terminology and implications for saving water and increasing production. Agric. Water Manage. 98, 1840-1846.

Perry, C., Steduto, P., Karajeh, F., 2017. Does improved irrigation technology save water? A review of the evidence. Discussion paper on irrigation and sustainable water resources management in the Near East and North Africa Food and. Agricultural Organization of the United Nations, Cairo, Egypt (accessed 30 April 2020). http:// www.fao.org/3/I7090EN/i7090en.pdf.

Peters, R.T., Evett, S.R., 2008. Automation of a center pivot using the Temperature-TimeThreshold method of irrigation scheduling. J. Irrig. Drain. Eng. 134 (3), 286-291.

Phogat, V., Skewes, M.A., McCarthy, M.G., Cox, J.W., Simunek, J., Petrie, P.R., 2017. Evaluation of crop coefficients, water productivity, and water balance components for wine grapes irrigated at different deficit levels by a sub-surface drip. Agric. Water Manage. 180, 22-34.

Playán, E., Salvador, R., López, C., Lecina, S., Dechmi, F., Zapata, N., 2014. Solid-set sprinkler irrigation controllers driven by simulation models: opportunities and bottlenecks. J. Irrig. Drain. Eng. 140. https://doi.org/10.1061/(ASCE)IR.1943-4774. 0000653.

Pôças, I., Calera, A., Campos, I., Cunha, M., 2020. Remote sensing for estimating and mapping single and basal crop coefficients: A review on spectral vegetation indices approaches. Agricultural Water Management 233, 106081. https://doi.org/10.1016/ j.agwat.2020.106081.

Pôças, I., Gonçalves, J., Costa, P.M., Gonçalves, I., Pereira, L.S., Cunha, M., 2017. Hyperspectral-based predictive modelling of grapevine water status in the Portuguese Douro wine region. Int. J. Appl. Earth Obs. 58, 177-190. https://doi.org/10.1016/j. jag.2017.02.013.

Popova, Z., Pereira, L.S., 2008. Irrigation scheduling for furrow irrigated maize under climate uncertainties in the Thrace Plain. Bulgaria. Biosyst. Eng. 99, 587-597.

Qiu, R., Song, J., Du, T., Kang, S., Tong, L., Chen, R., Wu, L., 2013. Response of evapotranspiration and yield to planting density of solar greenhouse grown tomato in northwest China. Agric. Water Manage. 130, 44-51.

Raes, D., Steduto, P., Hsiao, T.C., Fereres, E., 2017. AquaCrop version 6.0 reference manual. FAO, Rome, Italy (accessed 27 April 2020). http://www.fao.org/aquacrop.

Ramos, M.C., 2017. Effects of compost amendment on the available soil water and grape yield in vineyards planted after land levelling. Agric. Water Manage. 191, 67-76.

Ritchie, J.T., 1972. Model for predicting evaporation from a row crop with incomplete cover. Water Resour. Res. 8, 1204-1213.

Rizk, E.K., 2009. Effect of some anti-evaporative and anti-transpirant on water use efficiency of fig trees. Environ. Sci. Indian J. 4 (6), 403-411.

Rodrigues, G.C., Paredes, P., Gonçalves, J.M., Alves, I., Pereira, L.S., 2013. Comparing sprinkler and drip irrigation systems for full and deficit irrigated maize using multicriteria analysis and simulation modeling: ranking for water saving vs. farm economic returns. Agric. Water Manage. 126, 85-96. https://doi.org/10.1016/j.agwat. 2013.05.005.

Rodrigues, G.C., Pereira, L.S., 2009. Assessing economic impacts of deficit irrigation as related to water productivity and water costs. Biosyst. Eng. 103, 536-551.

Romero, P., Gil-Munoz, R., del Amor, F.M., Valdes, E., Fernandez, J.I., Martinez-Cutillas, A., 2013. Response of Vitis vinifera cv. 'Tempranillo' to partial rootzone drying in the field: water relations, growth, yield and fruit and wine quality. Agric. Water Manage. 121, 85-101.

Rosa, R.D., Paredes, P., Rodrigues, G.C., Alves, I., Fernando, R.M., Pereira, L.S., Allen, R.G., 2012a. Implementing the dual crop coefficient approach in interactive software. 1. Background and computational strategy. Agric. Water Manage. 103, 8-24. https:// doi.org/10.1016/j.agwat.2011.10.013.

Rosa, R.D., Paredes, P., Rodrigues, G.C., Fernando, R.M., Alves, I., Pereira, L.S., Allen, R.G., 2012b. Implementing the dual crop coefficient approach in interactive software. 2. Model testing. Agric. Water Manage. 103, 62-77. https://doi.org/10.1016/j. agwat.2011.10.018.

Rose, M.T., Patti, A.F., Little, K.R., Brown, A.L., Jackson, W.R., Cavagnaro, T.R., 2014. A Meta-analysis and review of plant-growth response to humic substances: practical implications for agriculture. Adv. Agron. 124, 37-89.

Rouphael, Y., Franken, P., Schneider, C., Schwarz, D., Giovannetti, M., Agnolucci, M., De Pascale, S., Bonini, P., Colla, G., 2015. Arbuscular mycorrhizal fungi act as biostimulants in horticultural crops. Sci. Hortic. 196, 91-108.

Rudnick, D.R., Irmak, S., 2014. Impact of nitrogen fertilizer on maize evapotranspiration crop coefficients under fully irrigated, limited irrigation, and rainfed settings. J. Irrig. Drain. Eng. 140 (12), 04014039.

Russo, V.M., Díaz-Pérez, J.C., 2005. Kaolin-based particle film has no effect on physiological measurements, disease incidence or yield in peppers. HortSci. 40, 98-101.

Ruzzi, M., Aroca, R., 2015. Plant growth-promoting rhizobacteria act as biostimulants in horticulture. Sci. Hortic. 196, 124-134.

Saglam, M., Sintim, H.Y., Bary, A.I., Miles, C.A., Ghimire, S., Inglis, D.A., Flury, M., 2017. Modeling the effect of biodegradable paper and plastic mulch on soil moisture dynamics. Agric. Water Manage. 193, 240-250.

Schiattone, M.I., 2018. Interventi agronomici per migliorare la produzione, la qualità e l'efficienza d'uso dell'acqua e dell'azoto della ruchetta [Diplotaxis tenuifolia (L.) DC.]". Università degli Studi della Basilicata, Potenza, Italy, pp. 191 PhD Thesis.

Schwartz, R.C., Baumhardt, R.L., Evett, S.R., 2010. Tillage effects on soil water 
redistribution and bare soil evaporation throughout a season. Soil Till. Res. 110, 221-229.

Shaheen, S.M., Hooda, P.S., Tsadilas, C.D., 2014. Opportunities and challenges in the use of coal fly ash for soil improvements: A review. J. Environ. Manage. 145, 249-267.

Sharda, R., Mahajan, G., Siag, M., Singh, A., Chauhan, B.S., 2017. Performance of dripirrigated dry-seeded rice (Oryza sativa L.) in South Asia. Paddy Water Environ. 15, 93-100.

Smith, R.J., Uddin, M.J., 2020. Selection of flow rate and irrigation duration for high performance bay irrigation. Agric. Water Manage. 228, 105850.

Snyder, R.L., de Melo-Abreu, J.P., 2005. Frost protection: Fundamentals, practice and economics. FAO, Rome, Italy.

Sohi, S.P., Krull, E., Lopez-Capel, E., Bol, R., 2010. A review of biochar and its use and function in soil. Adv. Agron. 105, 47-82. https://doi.org/10.1016/S0065-2113(10) 05002-9.

Spinoni, J., Barbosa, P., Bucchignani, E., Cassano, J., Cavazos, T., Christensen, J.H., Christensen, O.B., Coppola, E., Evans, J., Geyer, B., Giorgi, F., Hadjinicolaou, P., Jacob, D., Katzfey, J., Koenigk, T., Laprise, R., Lennard, C.H., Levent Kurnaz, M., Li, D., Llopart, M., McCormick, N., Naumann, G., Nikulin, G., Ozturk, T., Panitz, H.-J., Porfirio da Rocha, R., Rockel, B., Solman, S.A., Syktus, J., Tangang, F., Teichmann, C., Vautard, R., Vogt, J.V., Winger, K., Zittis, G., Dosio, A., 2020. Future global meteorological drought hot spots: A study based on CORDEX data. J. Clim. 33, 3635-3661.

Stanghellini, C., 2014. Horticultural production in greenhouses: efficient use of water. Acta Hort. 103, 25-32.

Stetson, L.E., Mecham, B.Q. (Eds.), 2011. Irrigation. 6th Edition. Irrigation Association. Fairfax, VA, USA.

Sui, R., Fisher, D.K., 2015. Field test of a center pivot irrigation system. Appl. Eng Agric. 31 (1), 83-88.

Tadayonnejad, M., Mosaddeghi, M.R., Ghorbani Dashtaki, Sh., 2017. Changing soil hydraulic properties and water repellency in a pomegranate orchard irrigated with saline water by applying polyacrylamide. Agric. Water Manage. 188, 12-20.

Tanner, C.B., Sinclair, T.R., 1983. Efficient water use in crop production: research or research? In: Taylor, H.M., Jordan, W.R., Sinclair, T.R. (Eds.), Limitations to Efficiency Water Use in Crop Production. Am. Soc. Agron., Madison, Wisconsin, USA, pp. $1-27$.

Tanny, J., 2013. Microclimate and evapotranspiration of crops covered by agricultural screens: A review. Biosyst, Eng. 114, 26-43.

Tarjuelo Martin-Benito, J.M., 2005. El Riego por Aspersión y su Tecnonlogía, 3rd ed.). Mundi-Prensa, Madrid in Spanish.

Thoreson, B., Lal, D., Clark, B., 2013. Drip irrigation impacts on evapotranspiration rates in California's San Joaquin valley. In: Wahlin, B.T., Anderson, S.S. (Eds.), Using 21st Century Technology to Better Manage Irrigation Water Supplies. USCID, Phoenix, Arizona, USA, pp. 155-169.

Tiercelin, J.R., Vidal, A. (Eds.), 2006. Traité d'Irrigation, 2nd ed. Lavoisier, Paris, France.

Todd, R.W., Klocke, N.L., Hergert, G.W., Parkhurst, A.M., 1991. Evaporation from soil influenced by crop shading, crop residue, and wetting regime. Trans. ASAE. 34 (2), 461-466.

Tripathi, V.K., Rajput, T.B.S., Patel, N., 2014. Performance of different filter combinations with surface and subsurface drip irrigation systems for utilizing municipal wastewater. Irrig. Sci. 32, 379-391.

Trout, T.J., Kincaid, D.C., 1989. Border cablegation system design. Transactions of the ASAE 32 (4), 1185-1192.

Ud-deen, M.M., 2009. Effect of plant growth regulators on growth and yield of mukhi kachu. Bangladesh J. Agric. Res. 34, 233-238.

Unger, P.W., Parker, J.J., 1976. Evaporation reduction from soil with wheat, sorghum, and cotton residues. Soil Sci. Soc. Am. J. 40 (6), 938-942.

Valín, I., Cameira, M.R., Pereira, L.S., 2012. Depivot: A model for center-pivot design and evaluation. Comput. Electron. Agric. 87, 159-170.

Vanuytrecht, E., Raes, D., Willems, P., Geerts, S., 2012. Quantifying field-scale effects of elevated carbon dioxide concentration on crops. Clim. Res. 54, 35-47.

Venot, J.-Ph., Kuper, M., Zwarteveen, M. (Eds.), 2019. Drip Irrigation for Agriculture. Untold Stories of Efficiency, Innovation and Development. Routledge, UK, pp. 358.

Vuolo, F., D’Urso, G., De Michele, C., Bianchi, B., Cutting, M., 2015a. Satellite-based irrigation advisory services: A common tool for different experiences from Europe to Australia. Agric. Water Manage. 147, 82-95.

Vuolo, F., Essl, L., Atzberger, C., 2015b. Costs and benefits of satellite-based tools for irrigation management. Front. Enviro. Sci. 3. https://doi.org/10.3389/fenvs.2015. 00052.

Walker, W.R., Skogerboe, G.V., 1987. Surface Irrigation. Theory and Practice. PrenticeHall, Englewood Cliffs, USA.

Wang, T., Melton, F.S., Pôças, I., Thao, T., Post, K., Johnson, L.F., Cassel-Sharma, F., 2020 Evaluation of crop coefficient and evapotranspiration data for sugar beets from Landsat surface reflectances using micrometeorological measurements and weighing lysimetry. Agric. Water Manage submitted for publication.

Wang, X., Ren, Y., Zhang, S., Chen, Y., Wang, N., 2017. Applications of organic manure increased maize (Zea mays L.) yield and water productivity in a semi-arid region. Agric. Water Manage. 187, 88-98.

Wei, Z., Paredes, P., Liu, Y., Chi, W.-W., Pereira, L.S., 2015. Modelling transpiration, soil evaporation and yield prediction of soybean in North China Plain. Agric. Water Manage. 147, 43-53.

Wen, Y., Shang, S., Yang, J., 2017. Optimization of irrigation scheduling for spring wheat with mulching and limited irrigation water in an arid climate. Agric. Water Manage. 192, 33-44.

Wright, J.L., 1982. New evapotranspiration crop coefficients. J. Irrig. Drain. Div. ASCE 108, 57-74.

Yacoubi, S., Zayani, K., Slatni, A., Playán, E., 2012. Assessing Sprinkler Irrigation Performance Using Field Evaluations at the Medjerda Lower Valley of Tunisia. Engineering 4, 682-691.

Yan, N., Wu, B., Perry, C., Zeng, H., 2015. Assessing potential water savings in agriculture on the Hai Basin plain. China. Agric. Water Manage. 154, 11-19.

Yang, J., Ma, X., Wang, K., Yang, W., 2018a. The coupled impact of plastic film mulching and deficit irrigation on soil water/heat transfer and water use efficiency of spring wheat in Northwest China. Agric. Water Manage. 201, 232-245.

Yang, Y., Ding, J., Zhang, Y., Wu, J., Zhang, J., Pan, X., Gao, C., Wang, Y., He, F., 2018b. Effects of tillage and mulching measures on soil moisture and temperature, photosynthetic characteristics and yield of winter wheat. Agric. Water Manage. 201, 299-308.

Yimam, A.Y., Assefa, T.T., Adane, N.F., Tilahun, S.A., Jha, M.K., Reyes, M.R., 2020. Experimental evaluation for the impacts of conservation agriculture with drip irrigation on crop coefficient and soil properties in the sub-humid Ethiopian Highlands. Water 12, 947. https://doi.org/10.3390/w12040947.

Zairi, A., El Amami, H., Slatni, A., Pereira, L.S., Rodrigues, P.N., Machado, T., 2003. Coping with drought: deficit irrigation strategies for cereals and field horticultural crops in Central Tunisia. In: Rossi, G., Cancelliere, A., Pereira, L.S., Oweis, T., Shatanawi, M., Zairi, A. (Eds.), Tools for Drought Mitigation in Mediterranean Regions. Kluwer, Dordrecht, pp. 181-201. https://doi.org/10.1007/978-94-0100129-8_10.

Zapata, N., Playán, E., Skhiri, A., Burguete, J., 2009. Simulation of a collective solid-set sprinkler irrigation controller for optimum water productivity. J. Irrig. Drain. Eng. 135, 13-24.

Zhang, D., Jiao, X., Du, Q., Song, X., Li, J., 2018. Reducing the excessive evaporative demand improved photosynthesis capacity at low costs of irrigation via regulating water driving force and moderating plant water stress of two tomato cultivars. Agric. Water Manage. 199, 22-33.

Zhang, X., Zhang, X., Liu, X., Shao, L., Sun, H., Chen, S., 2016. Improving winter wheat performance by foliar spray of ABA and FA under water deficit conditions. J. Plant Growth Regul. 35, 83-96. https://doi.org/10.1007/s00344-015-9509-6.

Zhao, N., Liu, Y., Cai, J., Paredes, P., Rosa, R.D., Pereira, L.S., 2013. Dual crop coefficient modelling applied to the winter wheat - summer maize crop sequence in North China Plain: basal crop coefficients and soil evaporation component. Agric. Water Manage. 117, 93-105.

Zhao, Y., Zhai, X., Wang, Z., Li, H., Jiang, R., Hill, R.L., Si, B., Hao, F., 2018. Simulation of soil water and heat flow in ridge cultivation with plastic film mulching system on the Chinese Loess Plateau. Agric. Water Manage. 202, 99-112.

Zribi, W., Araguiés, R., Medina, E., Faci, J.M., 2015. Efficiency of inorganic and organic mulching materials for soil evaporation control. Soil Till. Res. 148, 40-45. 